\title{
Rwanda: Request for a Three-Year Policy Support Instrument-Staff Report; Staff Supplement; Press Release on the Executive Board Discussion; and Statement by the Executive Director for Rwanda
}

In the context of the request for a three-year policy support instrument, the following documents have been released and are included in this package:

- $\quad$ The staff report for the Request for a Three-Year Policy Support Instrument, prepared by a staff team of the IMF, following discussions that ended on April 24, 2010, with the officials of Rwanda on economic developments and policies. Based on information available at the time of these discussions, the staff report was completed on May 28, 2010. The views expressed in the staff report are those of the staff team and do not necessarily reflect the views of the Executive Board of the IMF.

- $\quad$ A staff supplement of May 28, 2010 updating information on recent developments.

- $\quad$ A Press Release summarizing the views of the Executive Board as expressed during its June 16, 2010 discussion of the staff report that completed the request and/or review.

- $\quad$ A statement by the Executive Director for Rwanda.

The documents listed below have been or will be separately released.

Letter of Intent sent to the IMF by the authorities of Rwanda* Memorandum of Economic and Financial Policies by the authorities of Rwanda*

*Also included in Staff Report

The policy of publication of staff reports and other documents allows for the deletion of market-sensitive information.

\author{
Copies of this report are available to the public from \\ International Monetary Fund • Publication Services \\ $70019^{\text {th }}$ Street, N.W. • Washington, D.C. 20431 \\ Telephone: (202) 623-7430 • Telefax: (202) 623-7201 \\ E-mail: publications@imf.org Internet: http://www.imf.org
}

\section{International Monetary Fund Washington, D.C.}


INTERNATIONAL MONETARY FUND

RWANDA

\section{Request for a Three-Year Policy Support Instrument}

Prepared by the African Department

(In consultation with other departments)

Approved by Mark Plant and Christian Mumssen

May 28, 2010

- $\quad$ PSI request. The proposed three-year program aims to consolidate macroeconomic stability while reducing Rwanda's aid dependency by: (i) maintaining a sustainable fiscal position through improved revenue mobilization; (ii) strengthening monetary and exchange rate policies to ensure low and stable inflation; (iii) reducing financial sector vulnerability by strengthening banking supervision, and deepening the financial sector by enhancing access to credit; and (iv) diversifying the export base and improving the business environment. The policies underlying the request are summarized in the attached letter of intent and memorandum of economic and financial policies (MEFP).

- $\quad$ Program discussions. March 4-18, 2010 in Kigali and April 14-24, 2010 in Washington, D.C. Staff met with Minister of Finance and Economic Planning Rwangombwa, Governor of the National Bank of Rwanda Kanimba, heads of key government agencies, other officials, representatives of the private sector, and commercial banks. The team included Ms. McAuliffe (head), Mr. Davoodi, Mr. Opoku-Afari, and Ms. Sanya (EP) (all AFR), Mr. Geiregat (SPR), and was assisted by Mr. Gershenson (Resident Representative), and his local staff. Mr. Bartholomew (MCM) participated in the discussions. The mission overlapped with the World Bank.

- $\quad$ Fund relations. Three-year PRGF (now known as Extended Credit Facility (ECF)) arrangement for SDR 8.01 million (10.68 percent of quota) was approved on June 12, 2006, extended for two months in June 2009 and expired in August 2009. The Board concluded the sixth and final review under the PRGF on July 31, 2009. 


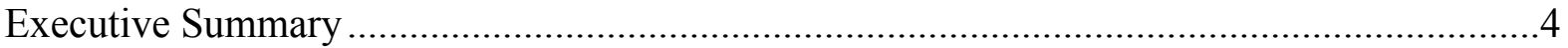

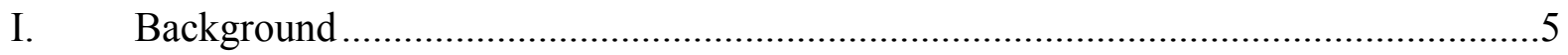

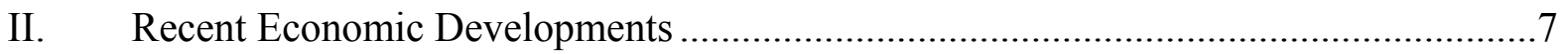

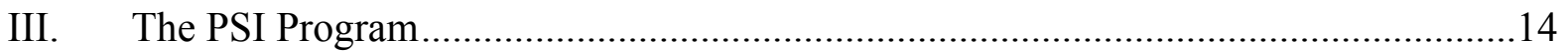

A. Transition to PSI

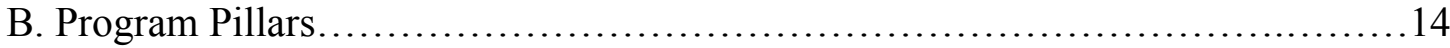

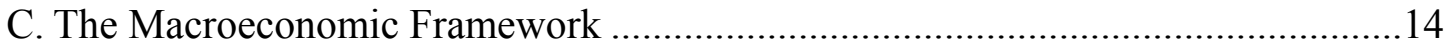

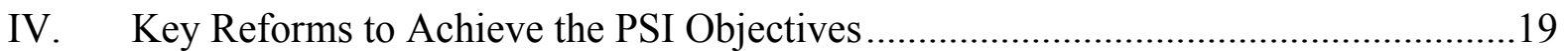

A. Ensuring Fiscal Consolidation and Long-Term Sustainability ..............................19

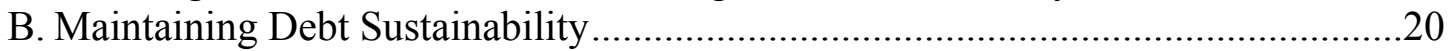

C. Ensuring Sufficient Policy Flexibility to Maintain Macroeconomic Stability ........21

D. Reducing Financial Sector Vulnerabilities and Improving Financial Depth ..........24

E. Improving the Business Environment and Enhancing Investment..........................24

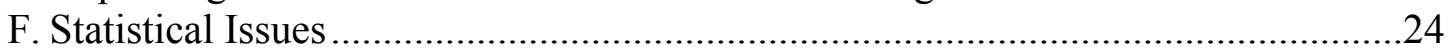

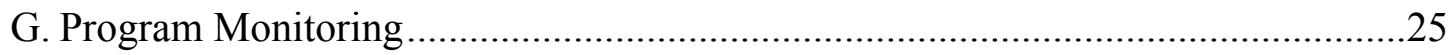

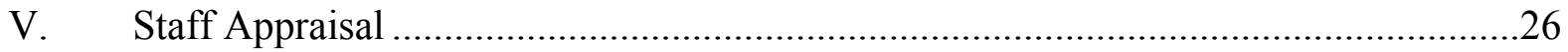

Tables

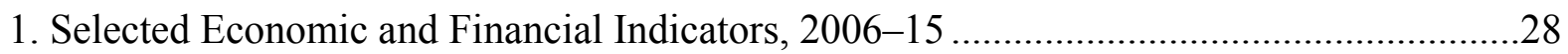

2. Operations of the Central Government, Fiscal Year Basis, 2006/07-14/15 ........................29

3. Monetary Survey, 2006-15 ...........................................................................................

4. Financial Soundness Indicators for Banking Sector, December 2006-December 2009 .....33

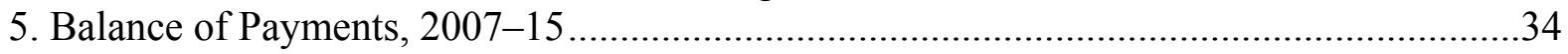

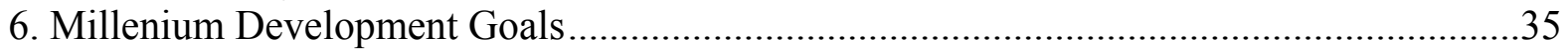

Figures

1. Recent Performance, Achievements and Challenges.........................................................

2. Selected Indicators of Economic Activity, Jan 2006-Feb 2010 …...................................... 8

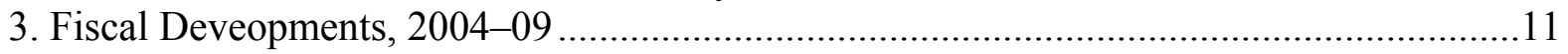

4. Monetary and Financial Developments, 2004-2009 ………….....................................12

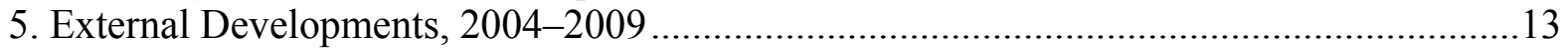

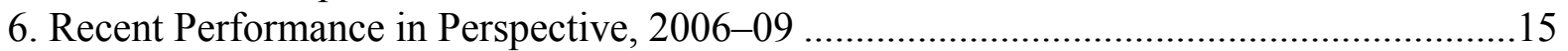

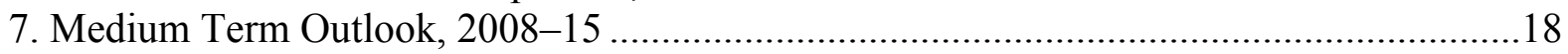


Boxes

1. Composite Index of Economic Activity (CIEA) for Rwanda. .23

2. Weaknesses in National Accounts Statistics.

Appendixes

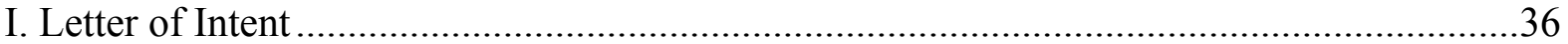

Attachment I. Rwanda: Memorandum of Economic and Financial Policies................38

Table 1. Quantitative Indicative Targets and Benchmarks for $2009 \ldots \ldots \ldots \ldots \ldots . . .58$

Table 2. Quantitative Assessment Criteria and Indicative targets for 2010/11 .....59

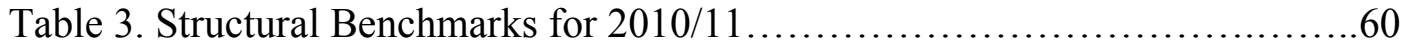

Attachment II. Rwanda: Technical Memorandum of Understanding........................61

Table 1. Summary of Reporting Requirements............................. 70 


\section{EXECUTIVE SUMMARY}

Rwanda has made significant progress under three successive PRGF arrangements, but important challenges remain. Rwanda remains a poor country, with a narrow export base, low government revenues, inadequate basic infrastructure, and heavily dependent on donor aid. The financial sector is shallow by regional standards, constrained by low domestic savings, and there are weaknesses in supervision. Rwanda has benefited from HIPC and MDRI debt relief.

The PSI addresses these policy challenges. The program supports the authorities' poverty reduction strategy while preserving macroeconomic stability and fiscal sustainability, strengthening monetary and exchange rate policies, and implementing growth enhancing structural reforms.

\section{Significant reforms are envisaged in each policy area:}

- $\quad$ Fiscal consolidation: front loaded by ambitious revenue mobilization efforts backed by significant revenue administration reforms, and a government-owned mediumterm PFM strategy.

- Monetary and exchange rate policies: underpinned by greater exchange rate flexibility and effective use of monetary policy instruments to deliver low and stable inflation.

- $\quad$ Structural reforms: tailored to address vulnerabilities arising from infrastructure bottlenecks, a narrow export base, and weak financial sector supervision capacity.

The authorities have an ambitious investment strategy aimed at alleviating critical infrastructure constraints to exports of goods and services and further enhancing competitiveness. The PSI accommodates some nonconcessional external borrowing tied to specific projects, without unduly increasing Rwanda's risk of debt distress. The borrowing takes into account the limited availability of concessional financing, a shallow domestic capital market, and the need to avoid crowding out the private sector

The medium-term economic outlook remains favorable. There are some risks but they appear manageable. Near term downside risks include slower-than-expected pick up in external demand and domestic credit conditions. Given Rwanda's comfortable level of reserves and prudent borrowing strategy, along with measures included in the PSI to improve debt management and further strengthen structural reforms, the risks can be managed to safeguard macroeconomic stability.

Staff supports the authorities' request for the PSI. 


\section{BACKGROUND}

1. Rwanda has achieved high growth and macroeconomic stability under three successive PRGF arrangements, but challenges remain. Real GDP growth averaged 8 percent a year during 1998-2008, up from 2 percent a year in the 1980s. Growth in Rwanda has been comparable to other countries in the region and higher than the average for SSA countries (Figure 1). Inflation, though volatile, is now in single digits, reserves are at comfortable levels, and external debt has been reduced significantly thanks to prudent policies and substantial debt relief. ${ }^{1}$ Nevertheless, Rwanda remains a poor country, with a narrow export base, low government revenues, inadequate basic infrastructure, and large development

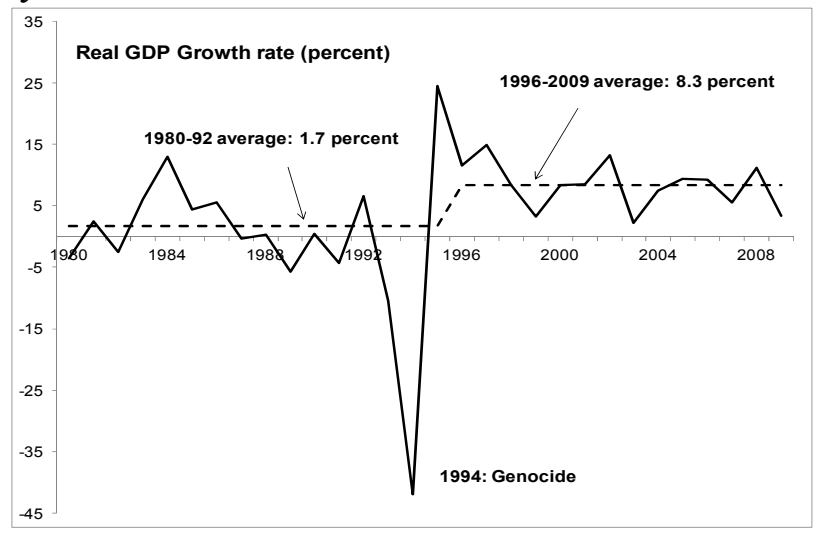
needs. The financial sector remains shallow by regional standards, and there are weaknesses in supervision. Rwanda also remains heavily dependent on donor aid.

\section{The authorities have requested a three-year PSI to anchor macroeconomic} policies and support further reforms to ensure high and sustained broad-based growth and poverty reduction. The authorities believe that a PSI is the appropriate successor to the recently-expired PRGF arrangement ${ }^{2}$, given Rwanda's lack of need of financial support from the Fund and track record of prudent macroeconomic policies. The government also favors such an arrangement to maintain a close policy dialogue with the IMF and signal to development partners its commitment to sound policies. The proposed program's three-year time frame is broadly in line with the remaining implementation period of Rwanda's Economic Development and Poverty Reduction Strategy (EDPRS) (covering 2008-12). ${ }^{3}$

\footnotetext{
${ }^{1}$ See IMF Country Report No. 09/264 for a review of performance under the last ECF arrangement.

2 The sixth and final review under Rwanda's three-year ECF arrangement was completed in July 2009 (Country Report No. 09/264) and the arrangement expired in August 2009.

3 Rwanda's Economic Development and Poverty Reduction Strategy 2008-2012 (IMF Country Report No. 08/90) and the Joint Staff Advisory Note on Rwanda's Economic Development and Poverty Reduction Strategy 2008-2012 (IMF Country Report No. 08/91) were issued to the Executive Board on February 14, 2008. An Annual Progress Report is under preparation and is expected to be made available to the IMF and other partners before the second review of the PSI.
} 
Figure 1. Rwanda: Recent Performance, Achievements and Challenges

Real GDP growth has been robust...

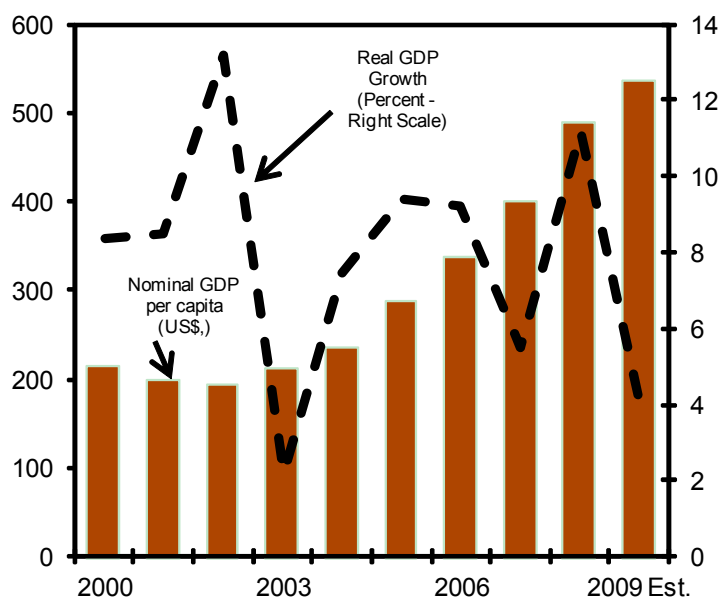

Inflation has returned to single digits while gross international reserves remain at

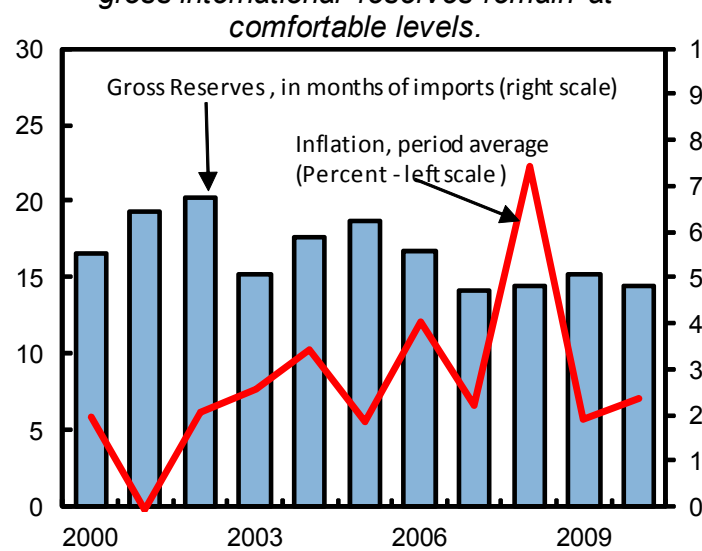

Rwanda remains a poor country...

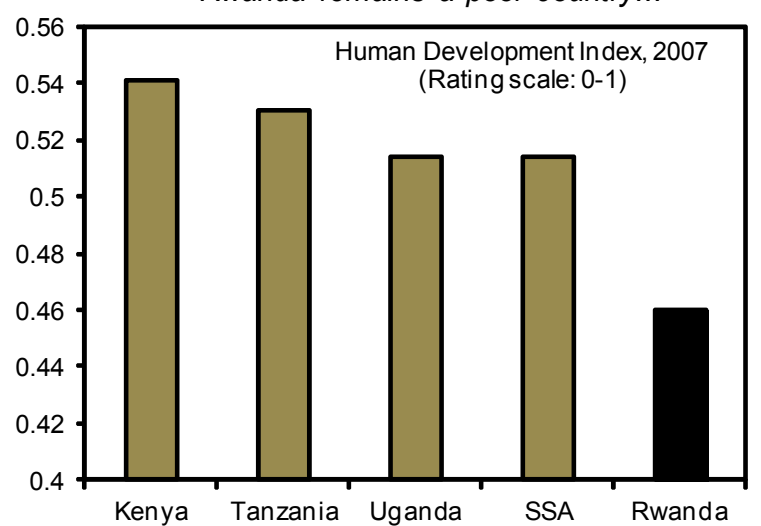

... comparable to other countries in the region and higher than the SSA average.

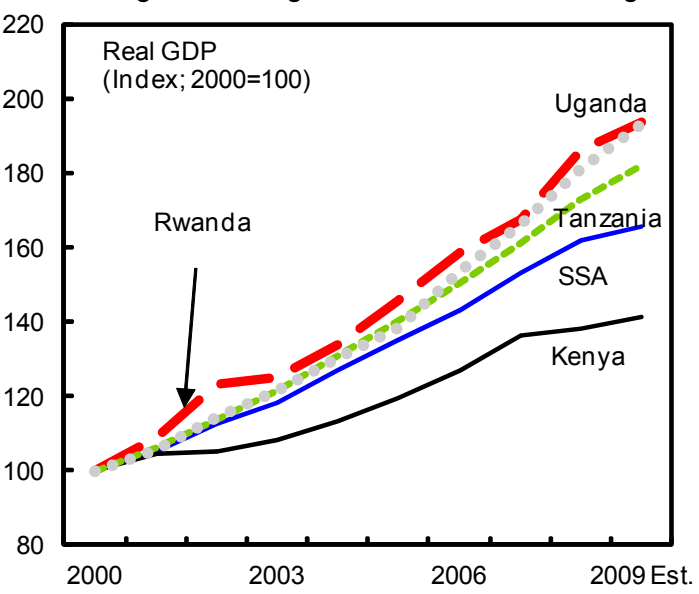

However, a narrow export base has contributed to large trade imbalances.

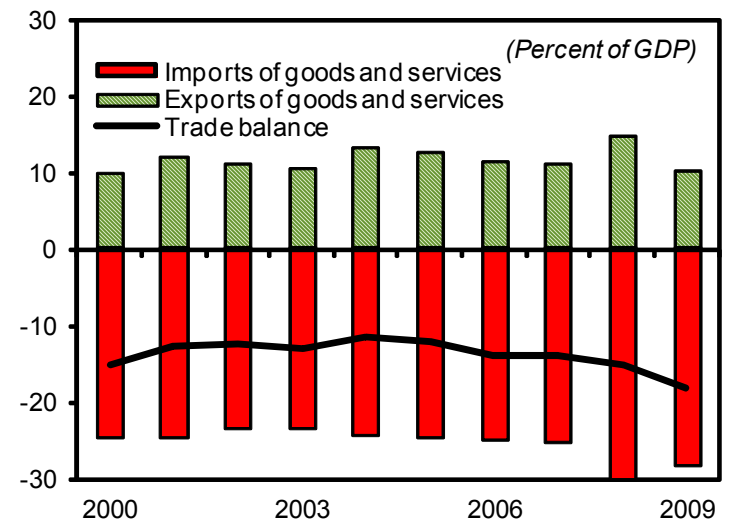

...that is heavily dependent on aid.

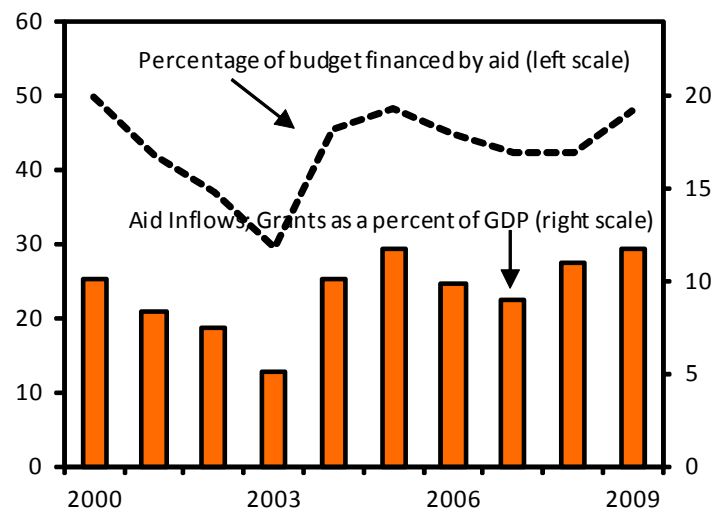

Source: Rwandan Authorities, IMF staff estimates, World economic Outlook, Regional Economic Outlook, and UNDP Human Development and Poverty Indicators. 


\section{RECENT ECONOMIC DEVELOPMENTS}

3. Growth slowed sharply in 2009, although staff and the authorities' views differ on the extent of the slow down, while inflation declined significantly (Table 1). After growing by 11.2 percent in 2008, staff estimates point to a slowdown in growth to 4 percent in 2009, compared with the authorities' estimate of 6 percent growth. Staff estimates are based on a more pronounced slowdown in wholesale and retail trade, construction, real estate, and manufacturing (Figure 2)-sectors that are particularly vulnerable to the global slowdown and tightened domestic liquidity conditions. Staff and the authorities agreed that the official GDP estimates are preliminary and subject to revision, while a number of methodological weaknesses in compilation of national accounts need to be addressed ( $\$ 25)$. Inflation in 2009 declined more rapidly than expected, from 22.3 percent in December 2008 to 5.7 percent in December 2009, reflecting lower world commodity prices and the slowdown in economic activity. Inflation declined further to 2 percent in March 2010 before edging upwards slightly to 3 percent in April, reflecting seasonal factors.

\section{Performance under the macro framework agreed with Fund} staff for 2009/10 has been broadly satisfactory. ${ }^{4}$ All but two indicative fiscal targets for end-December 2009 were met (MEFP Table 1). Net credit to the government was missed due to delays in disbursement of budgetary grants, which has since been disbursed. The zero ceiling on nonconcessional external borrowing was also missed due to a loan guarantee for the government-owned airline, Rwandair, one of the government's strategic investment projects. There were no structural benchmarks in the 2009/10 macro framework, but the authorities took significant steps to enhance revenue administration and public financial management, including by expediting the collection of tax arrears and a complete restructuring of the public procurement authority.

\footnotetext{
4 In the interim since the expiration of the PRGF arrangement, staff agreed with the authorities on a macroeconomic framework for 2009/10 (July-June) with indicative targets for monitoring purposes. The 2009/10 macro framework was revised in October 2009 taking into account changes in growth prospects, inflation outlook, and domestic liquidity conditions.
} 
Figure 2. Rwanda: Selected Indicators of Economic Activity, Jan 2006-Feb 2010
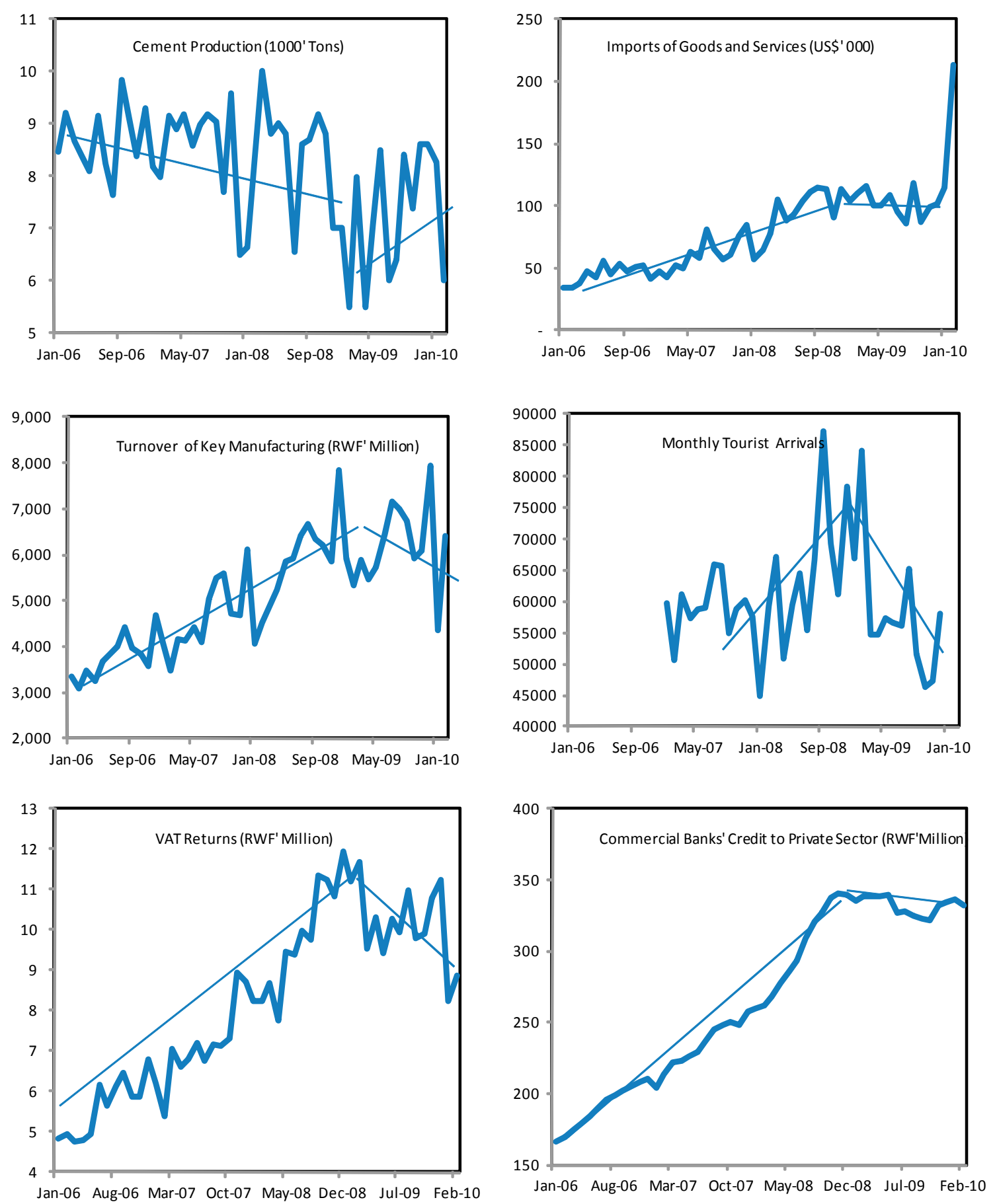

Source: IMF staff and Rwandan authorities estimates. 
5. Fiscal policy is continuing to inject a stimulus in $2009 / 10 .{ }^{5}$ The overall fiscal deficit (excluding grants) is expected to reach 13.7 percent of GDP in 2009/10, from 11.5 percent in 2008/09 and compared to 10 percent of GDP in 2007/08 and earlier, reflecting continued high levels of spending (Table 2 and Figure 3). Total expenditures and net lending is expected to remain unchanged at about 26 percent of GDP in 2009/10, up nearly 4 percent of GDP over the past 2 years. Net lending has increased as a share of GDP in 2009/10 owing to net lending to Rwandair and the Kigali International Convention Center project, two of the government's strategic investments. Domesticallyfinanced capital spending, on the other hand, has declined in 2009/10, owing to ongoing delays in completing project tender documents. Domestic revenues are expected to decline in $2009 / 10$ as a share of GDP. Total grants rose sharply higher-than-expected in 2009/10, mainly from higher budgetary grants in response to the global economic crisis.

6. Monetary policy was accommodative in $\mathbf{2 0 0 9}$ and so far this year. Significant progress was made in improving monetary management MEFP $q 10-12$ as both within-month and end-period reserve money targets were met in 2009 (Figure 4). Broad money growth (12-month) declined to 13 percent in 2009, compared to growth rates in excess of 20 percent in the previous three years, reflecting a sharp decline in credit growth to the private sector (Table 3). Following a period of tightened domestic liquidity, growth in credit to the private sector declined from 73 percent in 2008 to 5.7 percent in 2009. Bank liquidity improved during the year, as real deposit interest rates turned positive (for the first time in three years), though commercial banks remained cautious in extending credit amid a recent uptick in non-performing loans (Table 4). The National Bank of Rwanda (NBR) lowered its policy rate twice for a cumulative 200 basis points since early 2009. In a further effort to deepen the domestic financial market, the NBR entered into a swap agreement with the IFC in November 2009, which could provide additional resources for long-term lending to the private sector. ${ }^{6}$

7. The overall balance of payments recorded a surplus in 2009, despite a widening in the current account deficit (Table 5 and

Figure 5). A decline in exports, reflecting both lower international prices and weak external demand for Rwanda's main products (coffee, tea, and minerals), as well as lower tourism revenues, contributed to a widening of the current account deficit (excluding grants) to

\footnotetext{
${ }^{5}$ Rwanda's fiscal year runs July 1-June 30.

${ }^{6}$ According to the agreement, IFC would make available foreign exchange resources (up to US\$50 million) to the NBR at a pre-determined exchange rate in exchange for local currency equivalent. The IFC intends to have bilateral agreements with local banks to lend the resources to the private sector at variable interest rates. The NBR guarantees foreign exchange resource availability to IFC for repayment of the loans and escrows these resources so that they are not considered part of free reserves available to the NBR (MEFP 917 ).
} 
17.3 percent of GDP in 2009, from 14.4 percent in 2008. In contrast, the financial account improved significantly, mainly from the SDR allocation, as well as higher private-sector foreign direct investment and private-sector external borrowing. Official reserves coverage increased to 5.1 months of imports. The Rwandan franc was broadly stable against the U.S. dollar throughout 2009. The higher interest rates on local currency deposits, as well as the pickup in capital flows and remittances limited demand by the private sector for foreign exchange. The franc depreciated by 2.2 percent against the U.S. dollar over the year with minimal within-year variation, while the real effective exchange rate depreciated by some 5.1 percent. 
Figure 3. Rwanda: Fiscal Developments, 2004-09

The fiscal deficit widened on account of rising spending to mitigate the downturn...

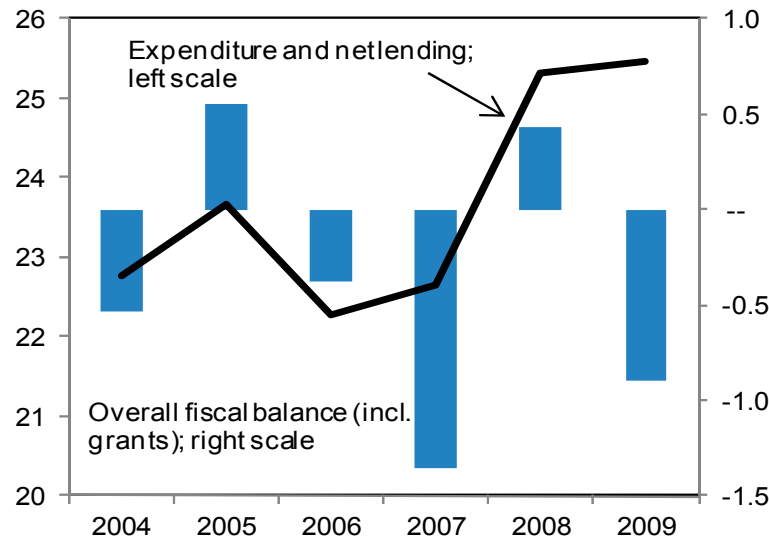

Priority spending increased in line with EDPRS priorities...

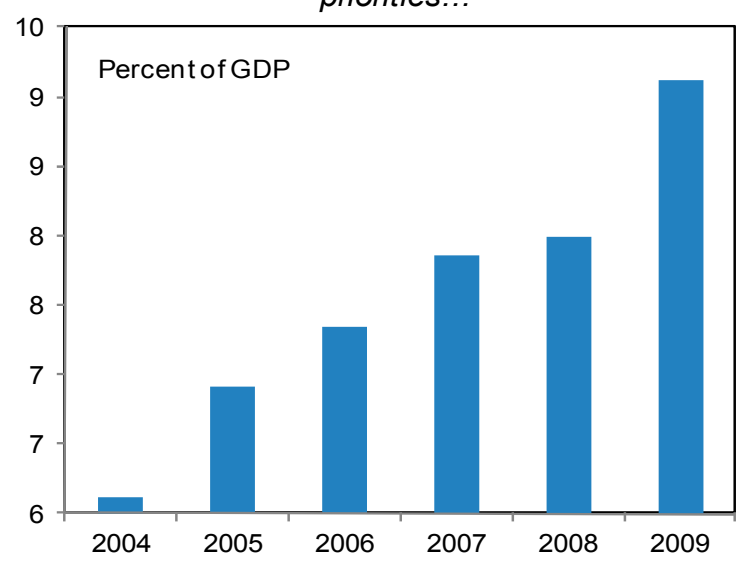

External debt is at low levels, thanks to substantial debt relief, ...

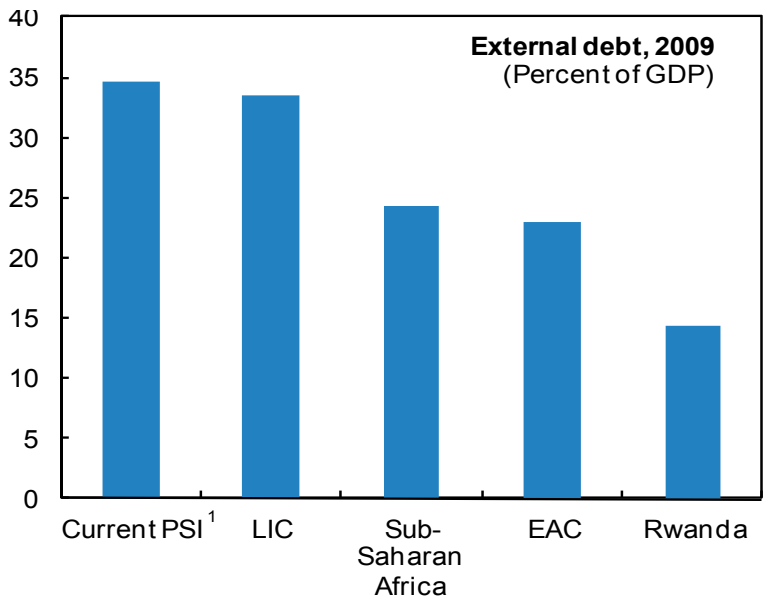

... as well as lower domestic revenues, while donor support remained strong.

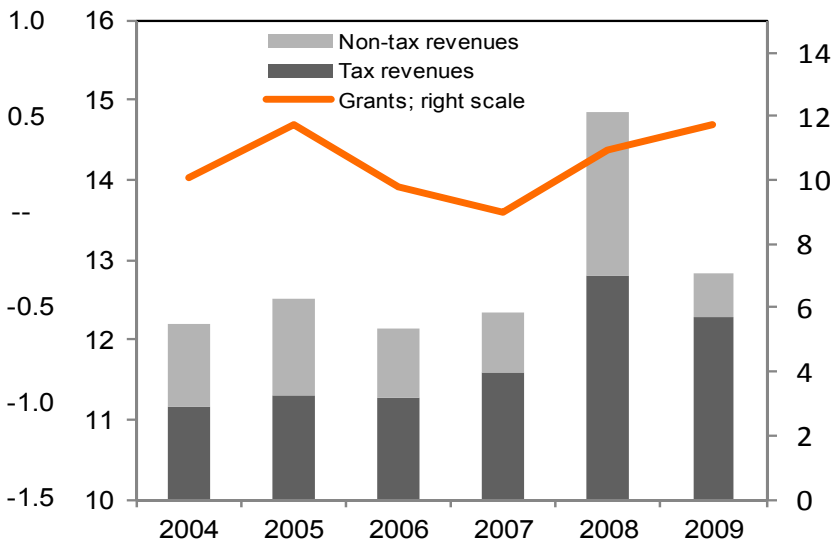

...while capital spending outpaced current spending by significant margins.

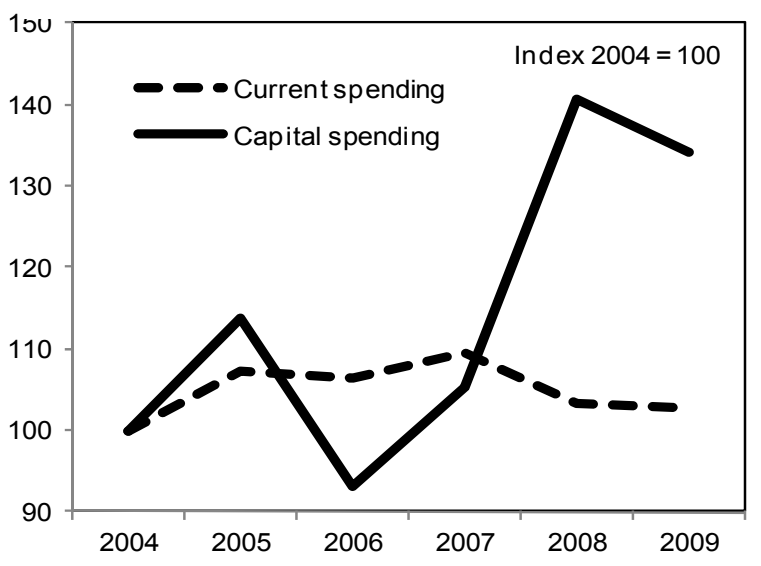

... while low domestic revenues remain a source of vulnerability.

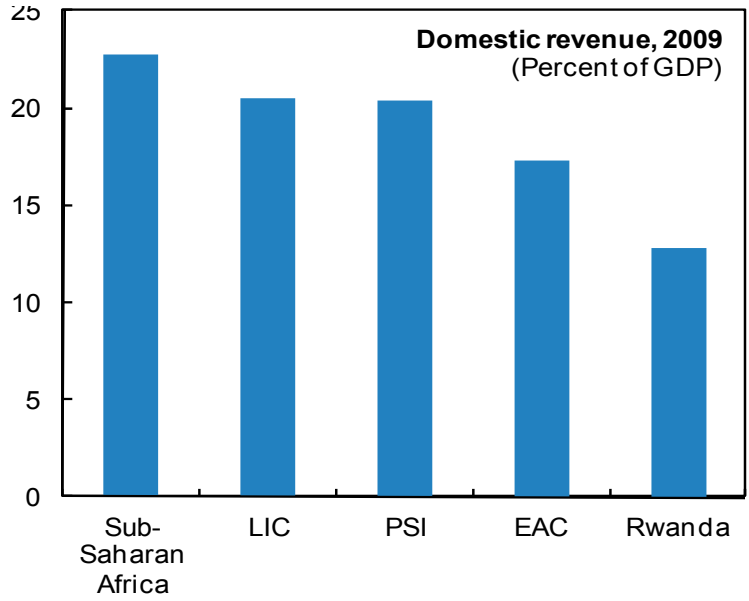

${ }^{1}$ Current PSI countries are: Cape Verde, Mozambique, Senegal, Tanzania, and Uganda.

Source: IMF staff and Rwandan authorities estimates. 
Figure 4. Rwanda: Monetary and Financial Developments, 2004-09

Accumulation of NFA has been a challengefor controlling reserve money...

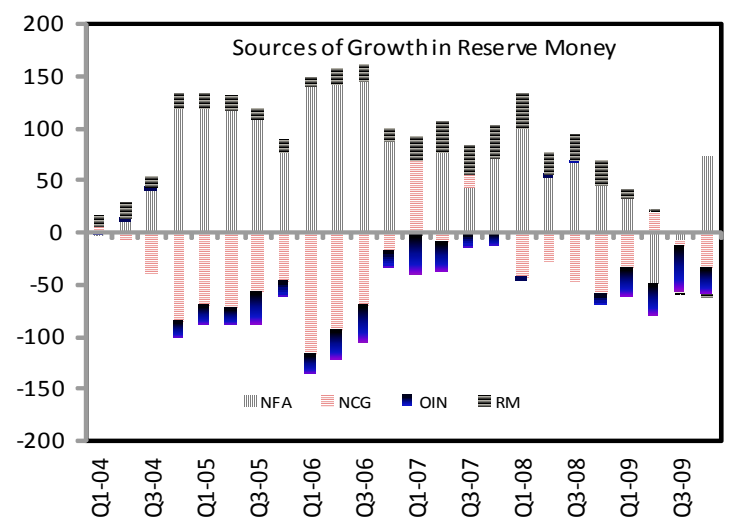

Prolonged period of negative real interest rates...

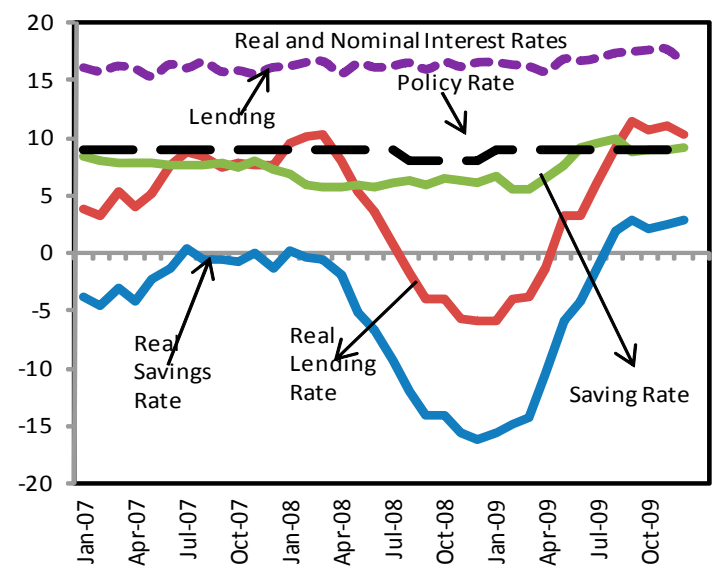

... and a sharp contraction in credit in 2009 which has started to turn around in 2010.

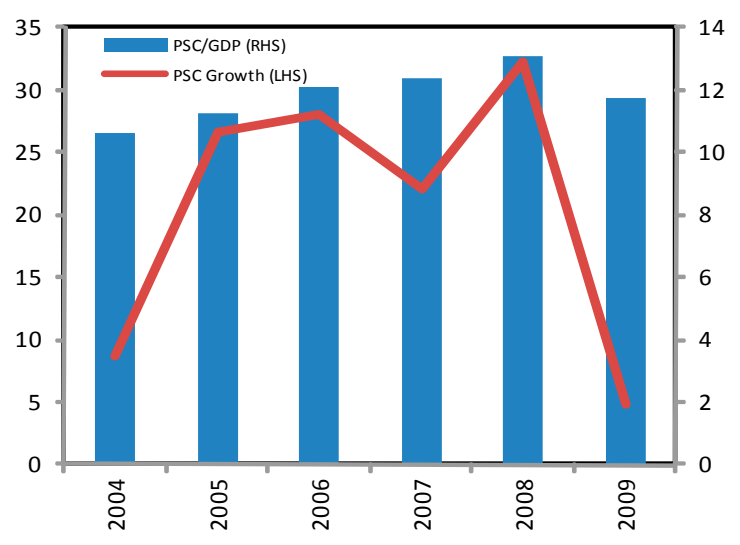

...however, control improved in 2009.

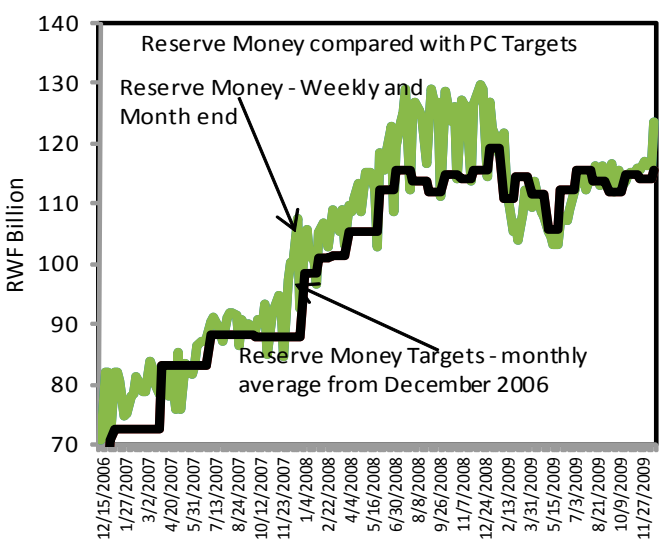

...led to sharp drops in deposits and liquidity tightening, which has since turned around...

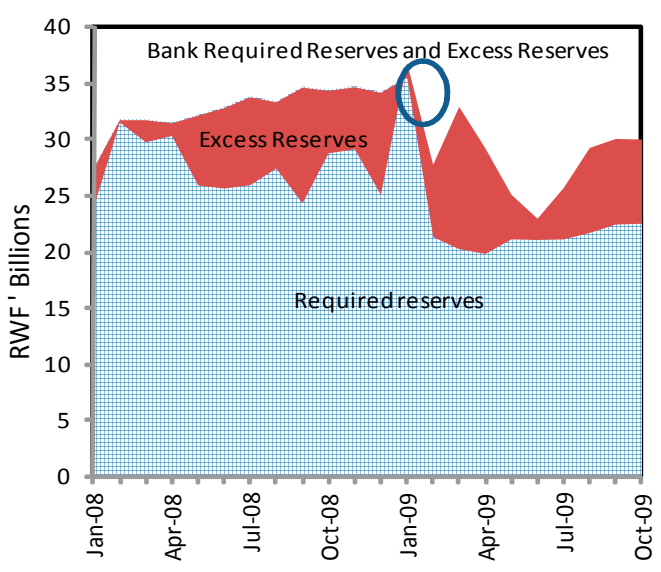

The banking system however, remains stable.

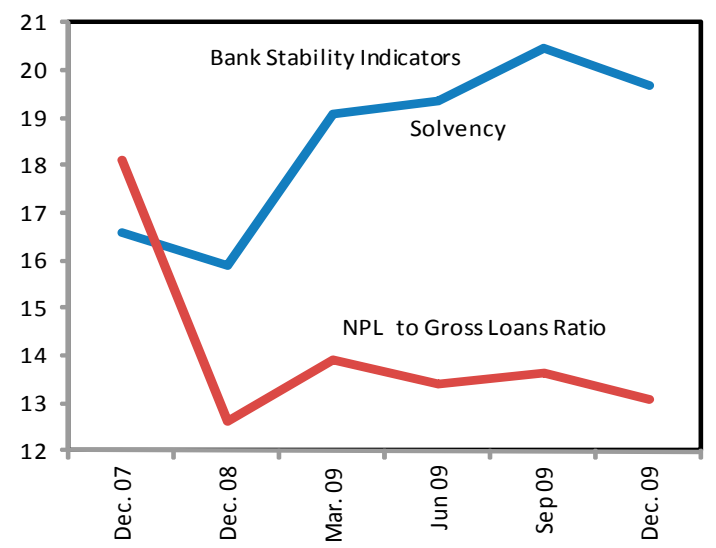

Source: IMF staff and Rwandan authorities estimates. 
Figure 5. Rwanda: External Developments, 2004-09

A downturn in mineral and tourism receipts hurt an already weak export base in 2009, while imports, especially capitalgoods, remain strong.

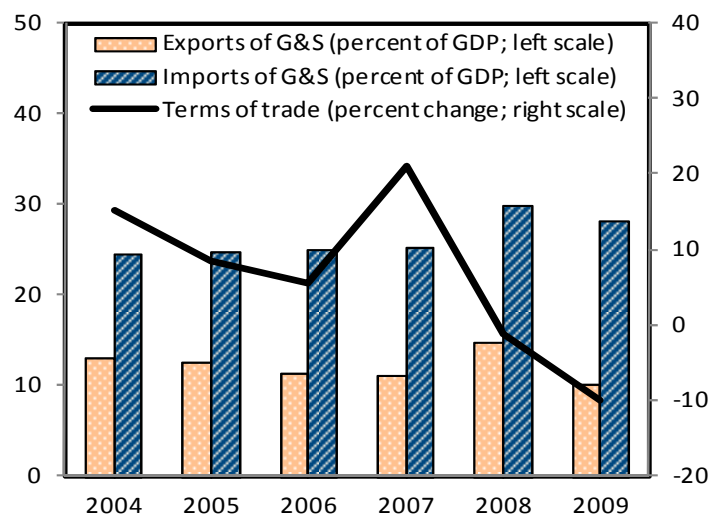

... in spite of higher transfers, especially from continued strong donor support.

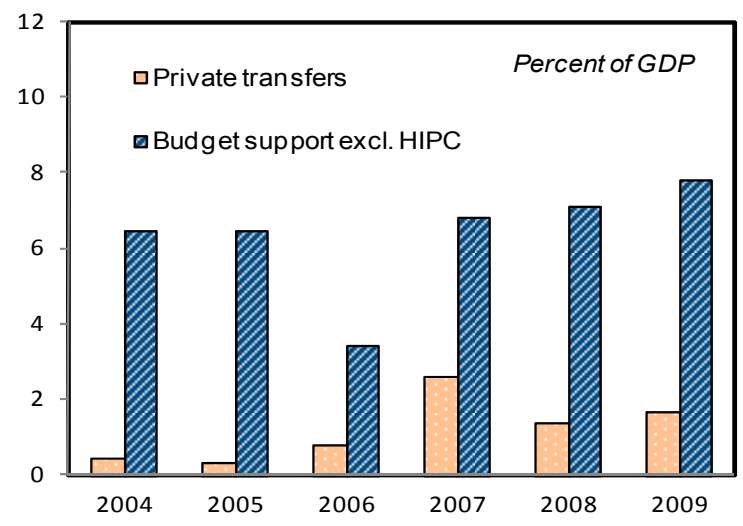

... and the overall reserves position has remained at comfortable levels.

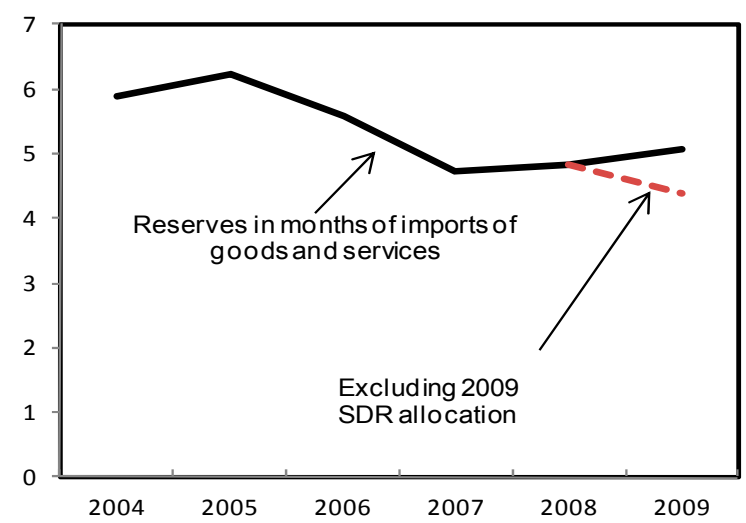

The current account deficit has continued to widen, ...

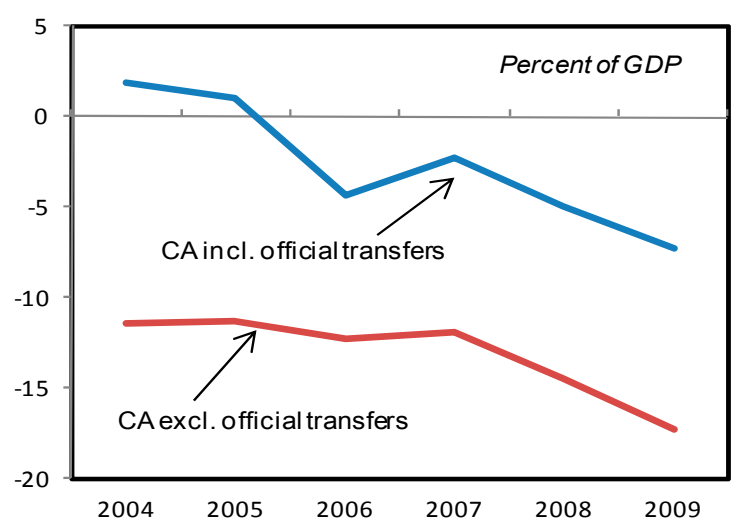

Increased foreign direct investment helps finance higherimports, ...

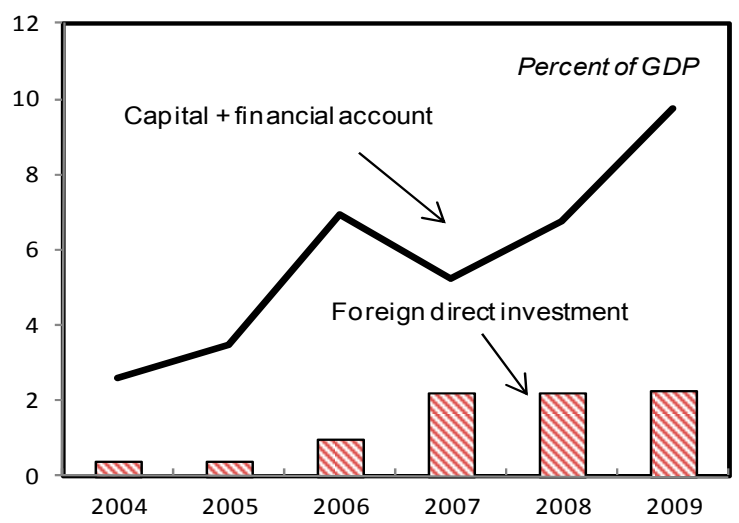

Since the depreciation in the second half of 2008, the franchas been traded in a narrow range.

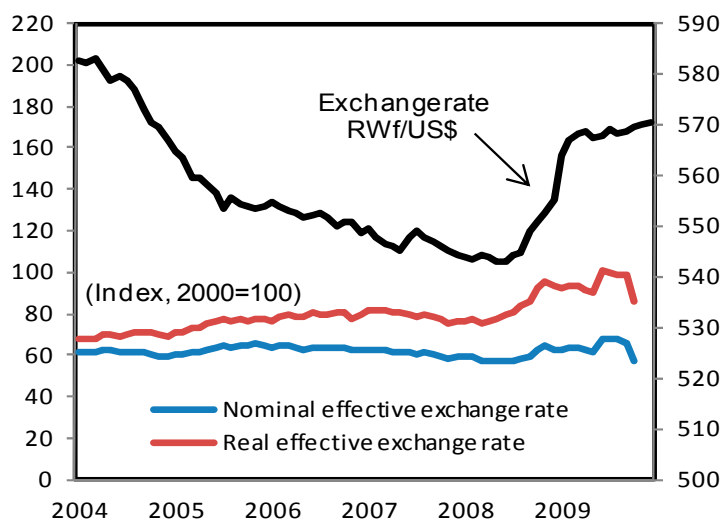

Source: IMF staff and Rwandan authorities estimates. 


\section{ThE PSI PrograM}

\section{A. Transition to PSI}

8. Rwanda's macroeconomic track record justifies transition to PSI-based Fund support. Over the past five years, real GDP growth has been very strong and macroeconomic management generally prudent, broadly in line with other PSI countries (Figure 6). This has contributed to low fiscal deficits, manageable current account deficits, and a comfortable level of international reserves. HIPC and MDRI debt relief has brought debt down, and the risk of debt distress is moderate. At the same time, inflation objectives have not always been met, reflecting some weaknesses in monetary and exchange rate policies. Moreover, Rwanda still faces significant vulnerabilities, in particular very low levels of fiscal revenues and exports, and high aid dependence. However, these vulnerabilities are mitigated by Rwanda's prudent policies, as demonstrated by its relatively strong economic performance during the global crisis and the absence of a need for Fund financing since 2002, beyond two low-access PRGF arrangements.

\section{B. Program Pillars}

9. The proposed PSI would support the authorities' program to achieve sustainable broad-based high growth and poverty reduction, in line with their EDPRS, while maintaining macroeconomic stability. Consistent with these objectives, discussions centered on four key pillars:

- maintaining a sustainable fiscal position, with increased revenue mobilization aimed at reducing Rwanda's aid dependency;

- $\quad$ strengthening monetary and exchange rate policies to ensure low and stable inflation;

- $\quad$ reducing financial sector vulnerabilities by strengthening banking supervision, and deepening the financial sector by enhancing access to credit; and

- $\quad$ underpinning growth with structural reforms to diversify the export base and improve the business environment.

\section{The Macroeconomic Framework}

10. The medium term macroeconomic framework reflects a gradual recovery after the slowdown in 2009. Real GDP growth is projected to increase gradually from 4.1 percent in 2009 to an annual average of about 6 percent in

2010-13 (Figure 7). This level of growth would be needed to lift per MEFP ๆ 22 capita incomes and reduce poverty. Growth is underpinned by a regional and global economic recovery, higher investment, and a more business-friendly environment that crowds in private investment. Credit to the private sector as a percent of GDP is 
projected to increase from 12.1 percent in 2009 to 14.5 percent in 2013 and stabilizes thereafter. Donor support is expected to remain strong, but decline gradually over the PSI period.

Figure 6. Rwanda: Recent Performance in Perspective, 2006-091
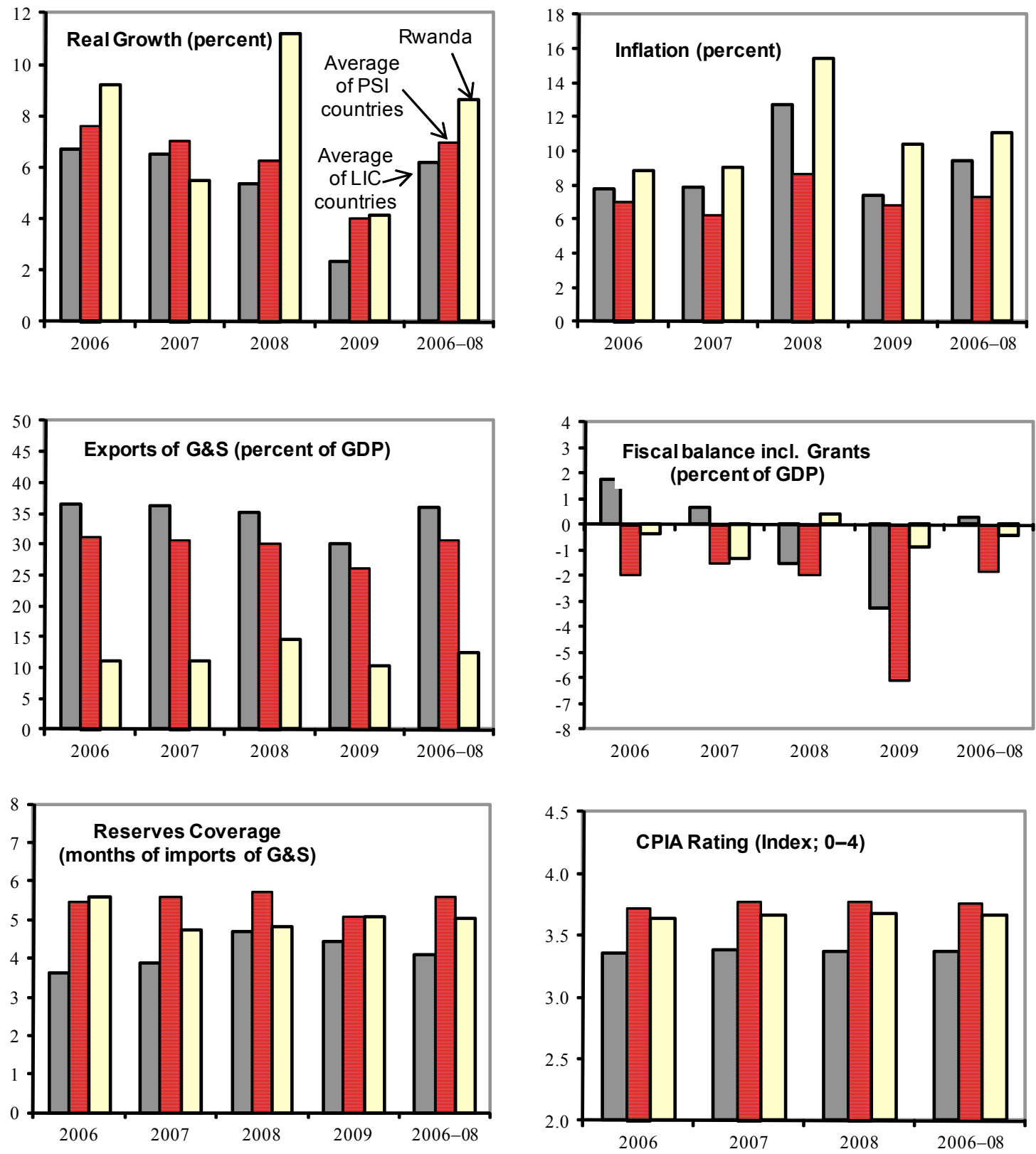

1 PSI Countries: Cape Verde, Mozambique, Nigeria, Senegal, Tanzania, and Uganda. Source: IMF, Fall 2009 WEO. For Rwanda, data are estimates for 2009. 
11. The authorities are targeting a higher growth path based on an ambitious investment strategy aimed at alleviating critical infrastructure constraints to exports of goods and services and further enhancing

MEFP 923

competitiveness. The bulk of the financing for these projects, however, is yet to be secured - the authorities are considering various funding mechanisms, including Public

Private Partnerships (PPPs) — and their implementation will likely extend beyond the medium term. In light of these uncertainties, staff and the authorities agreed to base the macroeconomic framework on a more conservative growth path over the medium term. There are downside risks to the outlook, in the near term from slower pickup in external demand and domestic credit, and greater uncertainty in donor flows and private financing in outer years. Political uncertainty surrounding upcoming presidential elections in August 2010 should be minimal.

\section{Achieving sustained growth without rekindling inflation requires a careful} balance of macroeconomic policies and structural reforms over the PSI period. The program envisages gradual fiscal consolidation, through revenue mobilization and unwinding the higher spending levels of recent years, given the prospect of declining grants and limited scope for domestic financing. Financial sector policies aimed at deepening the financial sector and enhancing efficient allocation of resources - aided by positive real interest rates on bank deposits - will increase domestic saving, investment, and growth. Monetary policy aided by a mix of greater exchange rate flexibility along with a more proactive use of the policy rate and sterilization instruments, will anchor inflationary expectations and help maintain a comfortable level of international reserves that cushion against exogenous shocks. Structural reforms aim at improving productivity and the business environment in order to help promote competitiveness and broaden the export base. The authorities will seek to continue to improve the living conditions of the poorest segments of the population, and meet the MDGs (Table 6), through prioritized spending, especially in health and education, in line with the EDPRS. 
Rwanda: Medium-Term Framework, 2007-15

\begin{tabular}{|c|c|c|c|c|c|c|c|c|c|}
\hline & 2007 & 2008 & $\begin{array}{c}2009 \\
\text { Est. }\end{array}$ & $\begin{array}{l}2010 \\
\text { Proj. }\end{array}$ & $\begin{array}{l}2011 \\
\text { Proj. }\end{array}$ & $\begin{array}{l}2012 \\
\text { Proj. }\end{array}$ & $\begin{array}{l}2013 \\
\text { Proj. }\end{array}$ & $\begin{array}{l}2014 \\
\text { Proj. }\end{array}$ & $\begin{array}{l}2015 \\
\text { Proj. }\end{array}$ \\
\hline & \multicolumn{9}{|c|}{ (Annual percent changes) } \\
\hline Real GDP & 5.5 & 11.2 & 4.1 & 5.4 & 5.9 & 6.4 & 6.9 & 6.5 & 6.5 \\
\hline \multirow[t]{2}{*}{ Consumer prices (p.a) } & 9.1 & 15.4 & 10.4 & 6.4 & 6.5 & 5.5 & 5.0 & 5.0 & 5.0 \\
\hline & \multicolumn{9}{|c|}{ (In percent of GDP, unless otherwise indicated) } \\
\hline Revenues $^{1}$ & 12.4 & 12.6 & 14.9 & 12.2 & 13.6 & 13.6 & 14.2 & 14.5 & 14.7 \\
\hline Grants $^{1}$ & 8.2 & 9.9 & 9.3 & 12.5 & 9.8 & 10.8 & 10.1 & 9.0 & 8.3 \\
\hline Government expenditure and net lending ${ }^{1}$ & 22.0 & 22.6 & 26.4 & 25.9 & 26.9 & 25.9 & 24.9 & 24.0 & 23.3 \\
\hline Overall fiscal balance (incl. grants) ${ }^{1}$ & -1.4 & -0.2 & -2.2 & -1.1 & -3.6 & -1.5 & -0.6 & -0.4 & -0.4 \\
\hline Current account balance (incl. official transfers) & -2.2 & -4.9 & -7.3 & -7.9 & -8.8 & -5.4 & -4.4 & -3.7 & -3.5 \\
\hline Gross official reserves (months of imports of $G \& S$ ) & 4.7 & 4.8 & 5.1 & 4.8 & 4.7 & 4.7 & 4.4 & 4.3 & 4.2 \\
\hline
\end{tabular}

${ }^{1}$ On a fiscal year basis (July-June). For example, the column ending in 2011 refer to FY2010/11. Other data are on a calendar year basis. Sources: Rwandan authorities; and IMF staff estimates and projections. 
Figure 7. Rwanda: Medium-term outlook, 2008-15

Output growth over the medium-term is projected to be driven by services and construction related to large investment projects.

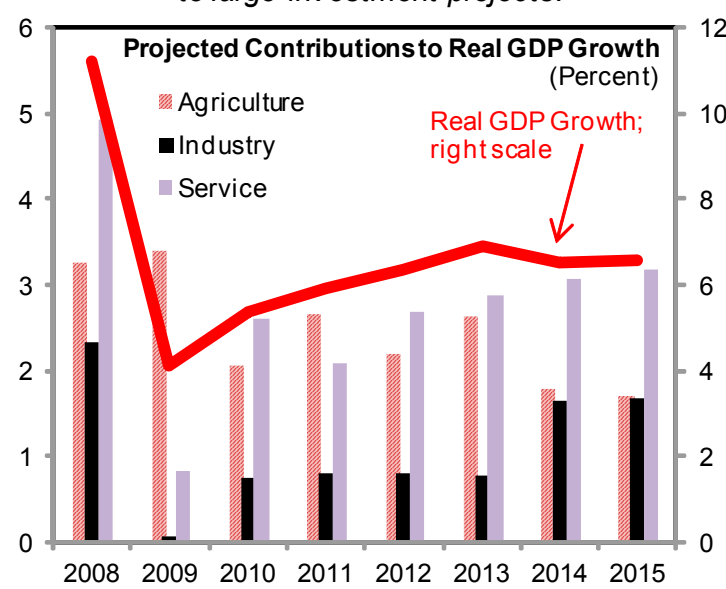

The external current account is expected to improve...

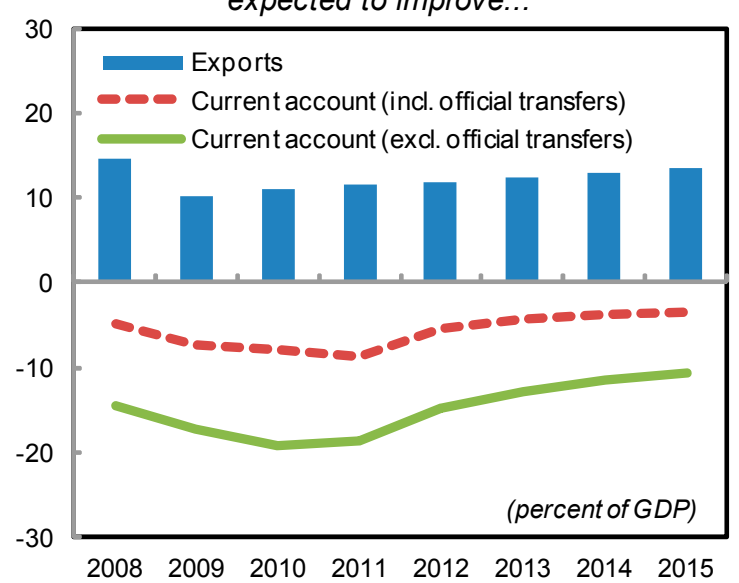

Gradual fiscal consolidation...

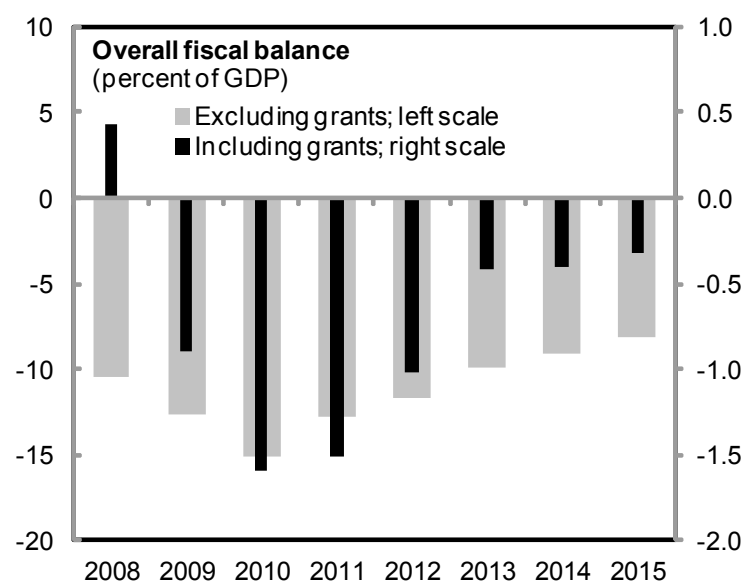

Inflation is expected to remain in single digits in the absence of renewed energy and food price pressures.

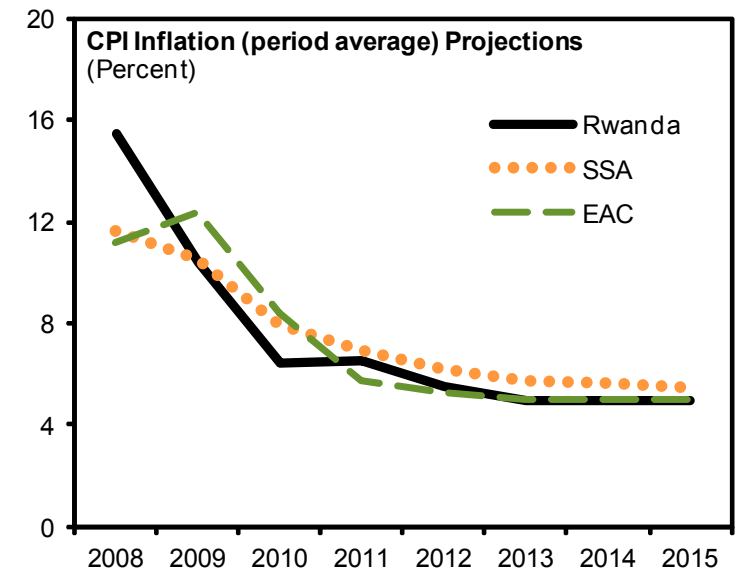

... while international reserves would remain at comfortable levels.

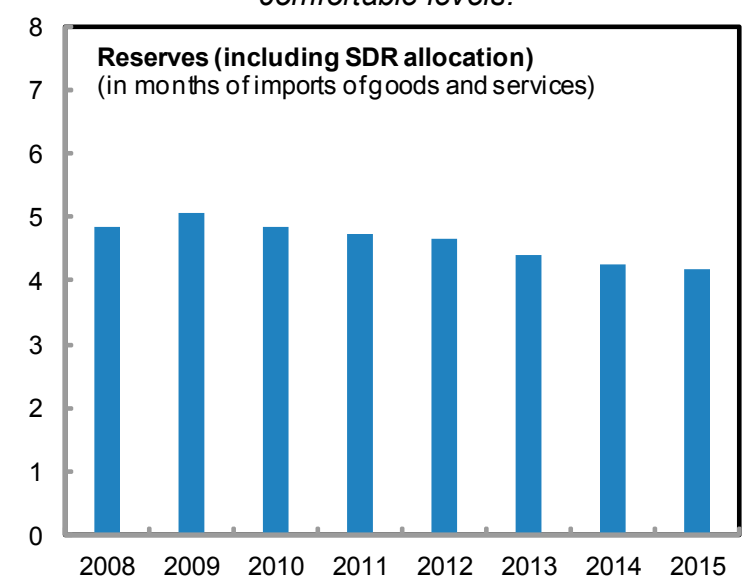

... is supported by higher domestic revenues and lower spending.

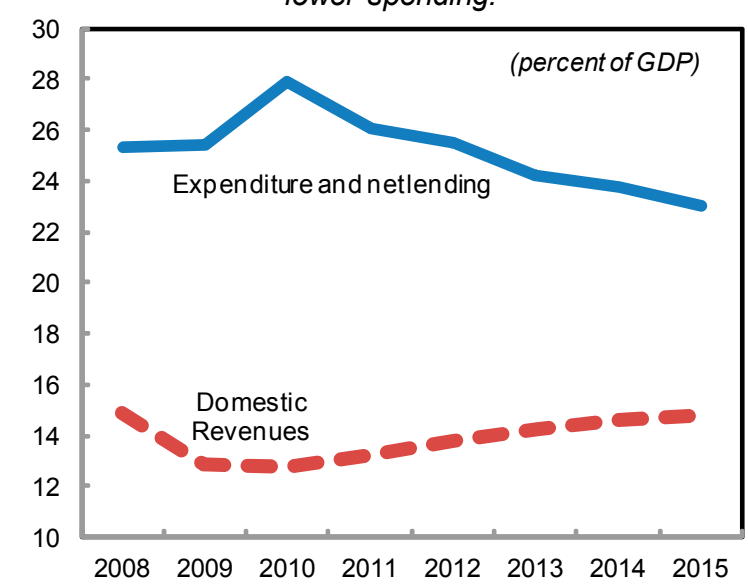

Source: IMF staff and Rwandan authorities estimates. 


\section{KeY REFORMS TO ACHIEVE THE PSI OBJECTIVES}

\section{A. Ensuring Fiscal Consolidation and Long-Term Sustainability}

\section{A reduction of the overall fiscal deficit (including grants) from $\mathbf{3 . 6}$ percent of} GDP in 2010/11 to less than 1 percent of GDP by 2012/13 is the cornerstone of the PSI program. According to the updated Debt Sustainability Analysis, the debt position is sustainable. The envisaged fiscal consolidation includes a gradual increase in revenues and decline in expenditure. Grant finance is projected to remain high over the PSI period though a gradual reduction is envisaged. The authorities' fiscal strategy also relies on significant PFM reforms in order to increase expenditure efficiency and value for money, particularly when a higher volume of borrowing may be needed to finance public investment.

\begin{tabular}{|c|c|c|c|c|c|c|c|c|}
\hline & $2007 / 08$ & $2008 / 09$ & $\begin{array}{r}2009 / 10 \\
\text { Proj. }\end{array}$ & $\begin{array}{r}2010 / 11 \\
\text { Proj. } \\
\end{array}$ & $\begin{array}{r}2011 / 12 \\
\text { Proj. } \\
\end{array}$ & $\begin{array}{r}2012 / 13 \\
\text { Proj. }\end{array}$ & $\begin{array}{r}2013 / 14 \\
\text { Proj. } \\
\end{array}$ & $\begin{array}{r}2014 / 15 \\
\text { Proj. }\end{array}$ \\
\hline & \multicolumn{8}{|c|}{ (In percent of fiscal year GDP) } \\
\hline Revenue and grants & 22.5 & 24.3 & 24.8 & 23.3 & 24.4 & 24.2 & 23.6 & 23.0 \\
\hline Revenues & 12.6 & 14.9 & 12.2 & 13.6 & 13.6 & 14.2 & 14.5 & 14.7 \\
\hline Grants & 9.9 & 9.3 & 12.5 & 9.8 & 10.8 & 10.1 & 9.0 & 8.3 \\
\hline Total expenditure and net lending & 22.6 & 26.4 & 25.9 & 26.9 & 25.9 & 24.9 & 24.0 & 23.3 \\
\hline Current expenditure & 15.1 & 14.5 & 14.9 & 14.6 & 15.2 & 15.4 & 15.3 & 15.3 \\
\hline Captial ependiture & 8.2 & 11.1 & 9.8 & 11.4 & 10.2 & 9.0 & 8.3 & 7.9 \\
\hline of which: Domestic & 3.8 & 5.1 & 4.8 & 6.3 & 5.3 & 5.2 & 5.1 & 5.0 \\
\hline Net lending & -0.7 & 0.8 & 1.2 & 0.9 & 0.5 & 0.5 & 0.3 & 0.2 \\
\hline \multicolumn{9}{|l|}{ Overall deficit (commitment basis) } \\
\hline After grants & -0.2 & -2.2 & -1.1 & -3.6 & -1.5 & -0.6 & -0.4 & -0.4 \\
\hline Before grants & -10.0 & -11.5 & -13.7 & -13.4 & -12.4 & -10.7 & -9.4 & -8.6 \\
\hline Change in arrears & -0.4 & -0.3 & -0.3 & -0.2 & -0.2 & -0.2 & -0.2 & -0.1 \\
\hline \multicolumn{9}{|l|}{ Overall deficit (cash) } \\
\hline After grants & -0.5 & -2.5 & -1.4 & -3.8 & -1.7 & -0.8 & -0.6 & -0.5 \\
\hline Before grants & -10.4 & -11.8 & -13.9 & -13.6 & -12.6 & -10.9 & -9.6 & -8.8 \\
\hline Financing & 0.5 & 2.5 & 1.4 & 3.8 & 1.7 & 0.8 & 0.6 & 0.5 \\
\hline Net external financing & 1.9 & 2.6 & 0.8 & 1.5 & 1.7 & 0.8 & 0.6 & 0.5 \\
\hline Net domestic financing & -1.4 & -0.1 & 0.6 & 2.3 & 0.0 & 0.0 & 0.0 & 0.0 \\
\hline
\end{tabular}

Sources: Rwandan authorities; and IMF staff estimates and projections.

14. The authorities agreed on the importance of increasing domestic revenues-by 2 percent of GDP — over the PSI period, backed up by significant revenue administration reforms. At 12.2 percent of GDP in 2009/10, Rwanda's revenues are well below SSA and regional averages. Higher revenues are MEFP $\{31,54$ needed to meet large spending needs, as well as lay the groundwork for reducing Rwanda's aid dependency over the long term. Revenue increases are front loaded in the first year of the PSI in order to broaden the revenue base for subsequent years. The revenue administration reforms envisaged in 2010/11 include transferring collection of social security contributions to the Rwanda Revenue Authority (RRA), improving RRA's debt management strategy (including collection of tax arrears), and strengthening risk-based assessment in the 
collection of customs duties. These measures are planned for the first half FY2010/11 and are expected to have immediate impact on widening the tax net by capturing all social security recipients and generating sustained higher revenues, including a sharp pickup in the second half of the fiscal year. Further revenue administration measures are planned over the medium term, including automating the collection and filing of taxes, including social security taxes.

15. The government intends to deepen PFM reforms, with emphasis on improving expenditure efficiency and monitoring priority expenditures. In 2010/11, key modules of the IFMIS (Integrated Financial Management Information System) will be piloted in five government agencies with an eye toward a full roll out to all Ministries and agencies pending a review of the pilot exercise. A Public Expenditure Review (PER) will be completed for two more sectors-with support from the World Bank - which would further improve expenditure efficiency and strengthen costing in the medium-term expenditure framework (MTEF). A PEFA update is planned for July-September 2010 which will inform future direction of PFM reforms. Over the medium term, further PFM reforms include: strengthening the MTEF process and the IFMIS, conducting PERs for more sectors and enhancing audit and external oversight of the budget process.

\section{B. Maintaining Debt Sustainability}

\section{The proposed PSI accommodates up to US\$240 million (4.7 percent of 2009} GDP) in nonconcessional external borrowing for the duration of the program. The nonconcessional borrowing would be tied to loan guarantees for Rwandair operations and the building of the Kigali

MEFP $\llbracket 39$ International Convention Complex, two of the Government's strategic investment projects. The authorities have conducted feasibility studies, with assistance from international consultants, which show these projects are expected to have rates of return above financing costs. ${ }^{7}$ The borrowing takes into account the limited availability of concessional financing, a shallow domestic capital market, and the need to avoid crowding out the private sector.

\section{An updated DSA indicates that Rwanda continues to have a moderate risk of debt}

distress. The baseline macroeconomic scenario indicates that Rwanda's debt dynamics would be sustainable even if the assumed amount of nonconcessional external borrowing is contracted. However, under stress tests, especially adverse shocks to exports, debt vulnerabilities increase, owing to the low export base.

\footnotetext{
${ }^{7}$ See the Debt Sustainability Analysis, a supplement to this report, for details.
} 
18. The authorities are mindful of the need to mitigate these risks. Two structural benchmarks during the first year of the PSI aim to lay the groundwork for diversifying exports and strengthening debt management. A draft export strategy and action plan will be submitted for Cabinet approval by August 2010. A MEFP 959 revised medium-term debt strategy (MTDS) that takes into account nonconcessional financing, as well as possible fiscal risks associated with PPPs, will be submitted to Cabinet by December 2010. The authorities plan to continue to build up capacity to conduct annual DSA's on their own. Staff strongly urged the authorities to finalize the ongoing work to establish a sound and legal monitoring framework for PPPs prior to entering into any arrangements. Further scaling up investments over the medium-term, including additional nonconcessional borrowing, could be considered in the context of PSI reviews, subject to an assessment of capacity constraints, demand pressures, and financing conditions.

\section{Ensuring Sufficient Policy Flexibility to Maintain Macroeconomic Stability}

\section{Monetary policy aims at achieving a 7 percent inflation target} at end-2010, taking into account expected rise in oil prices, and stabilizing it at 5 percent over the medium-term. Reserve money will continue to be the anchor of monetary policy, but with a shift towards targeting average monthly reserve money rather than end-month targets. The authorities have committed to making monetary policy more proactive by regularly reviewing the key repo rate to respond to changing fundamentals. To achieve this, NBR's Monetary Policy Committee (MPC) has increased the frequency of its meetings to quarterly. The NBR is also developing forecasting models and is compiling high-frequency data to support decision-making at the MPC (Box 1). In line with these commitments, the policy rate has lately become more flexible.

20. The NBR will enhance its communication strategy. It will

issue a report following each MPC meeting, explaining the monetary MEFP $q 43$ policy decisions and assessments of economic fundamentals underpinning these decisions. This is expected to help shape expectations of the market and the general public. 
21. The reform agenda in monetary and exchange rate policies also includes moving towards greater exchange rate flexibility and developing a more active interbank market. The NBR has taken measures to shift towards the use of an interbank foreign exchange corridor as a transition measure towards greater exchange rate flexibility. As a first step, in March 2010, NBR moved away from the use of an average reference rate (ARR) ${ }^{8}$ for intervention purposes. Since this measure was introduced, the exchange rate has been moving in line with market factors and there has been an increase in interbank activity with very minimum NBR interventions. Going forward, the NBR intends to intervene in the market only at the ends of the corridor to contain large short-term fluctuations. The corridor will be recalculated on a daily basis, in line with market MEFP $\uparrow 44$ transactions. This is aimed at encouraging trading among the banks in the interbank market within the corridor, as well as ensuring that the exchange rate moves in line with the economic fundamentals. The NBR is receiving TA from MCM in the design of the corridor, including the size and triggers for intervention.

\footnotetext{
${ }^{8}$ Average reference rate (ARR) is a 5-day moving average of the NBR selling rate for intervention purposes.
} 


\section{Box 1. A Composite Index of Economic Activity (CIEA) for Rwanda}

Lack of high frequency data for economic activity poses challenges for macroeconomic policy making. To address this weakness, the National Bank of Rwanda (NBR) has developed a Composite Index of Economic Activity (CIEA). The index of economic activity has been produced using available high frequency data that are correlated with GDP. Key variables used are: cement production, imports, exports, turnover of leading manufacturing establishments, VAT returns, and banking sector credit to the private sector. The monthly CIEA for January 2006 to February 2010 are shown below. The index tracks closely the strong growth in 2008 and slowdown in 2009. It could serve as a useful guide on the short-run dynamics of economic activity, as it closes the information gap and delays in computing the GDP outturn. Looking back, developments in 2008 (high growth and high inflation) show that the monetary policy stance in 2008 could have been tighter had the policymakers (particularly monetary policy authorities) had access to this index. As it turned out, the stronger growth in 2008 was only known to monetary policy authorities half way through 2009; at the time, there was not adequate information available to properly assess the balance of risks to position monetary policy to contain the underlying inflationary pressures.

Rwanda: Composite Index of Economic Activity, 2006-10
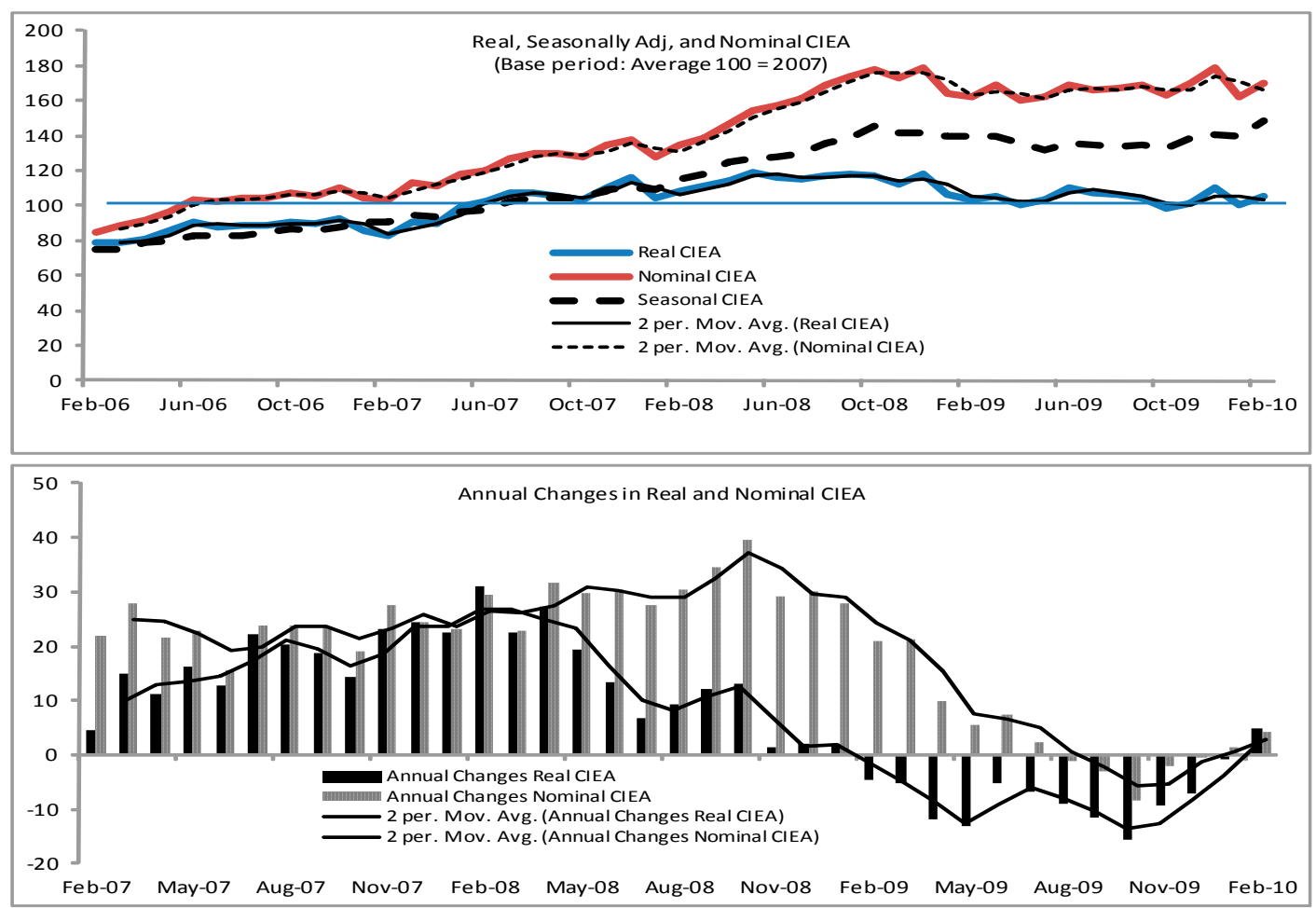


\section{Reducing Financial Sector Vulnerabilities and Improving Financial Depth}

22. The authorities are stepping up their efforts to address the vulnerabilities in the

financial sector. Supervision will be enhanced by recruiting, training, and

retaining bank examiners to keep up with both on-site and off-site

MEFP $₫ 49,57$ supervision consistent with the risk based supervision framework. Over the medium term, a comprehensive legal and regulatory framework will be put in place to support an increasingly diversified financial sector. The passage of a new pension law is expected to give de jure power to the NBR to proceed with supervision of the public pension fund (Caisse Sociale du Rwanda) and the insurance industry.

23. Further deepening of the financial sector is also underway. The central bank is in the process of licensing a private credit bureau aimed at enhancing the assessment and pricing of risks; and putting in place a comprehensive and integrated payments and settlement system to promote efficiency in financial MEFP $\mid 50-52$ transactions, consistent with the Financial Sector Development Plan (FSDP).

\section{E. Improving the Business Environment and Enhancing Investment}

24. The authorities recognize the importance of reforming the business environment to facilitate private sector activity, investment and strategically position Rwanda to benefit from regional integration. The authorities are taking measures to sustain the momentum of reforms which have yielded significant success in the 2010 Doing Business Indicators by rolling out key reforms on a MEFP 959 countrywide basis. ${ }^{9}$ Over the medium-term, reforms will aim to: (i) remove key bottlenecks and impediments including simplification of property registration procedures, establishing an automated cargo tracking system at the border and simplifying tax administration and information services, (ii) address shortages of skilled labor, as a potentially significant impediment to growth, (iii) expand the export base, by increasing value added of existing exports and developing new niche sectors, in line with the National Export Strategy and action plan.

\section{F. Statistical Issues}

25. The national statistical agency (NISR) is taking measures to address weaknesses in national accounts statistics (Box 2). Given the importance of agriculture and the informal sector in the economy, the NISR will seek MEFP $₫ 61-63$ technical assistance to ensure a proper design for the household survey and revise the methodology for conducting ex-ante and ex-post crop assessments. Over the

\footnotetext{
9 In September 2009, Rwanda was listed as the world's top reformer of business regulation according to the World Bank's Doing Business Index, the first time for a Sub-Saharan African economy. Rwanda's ranking in the 2010 index rose from $143^{\text {rd }}$ place to $67^{\text {th }}$ place.
} 
medium term, it intends to conduct a survey all enterprises so that GDP can be more accurately measured from the production side.

\section{Box 2. Weaknesses in National Accounts Statistics}

Despite recent advances in compilation of national accounts statistics, including the production of quarterly GDP figures, data quality suffers from weak data sources, limited survey design and methodological inconsistencies between quarterly and annual national accounts data:

- $\quad$ The size of agricultural sector, which is deemed to be large, is estimated from a pre-harvest crop assessment, but is not verified against actual volume of agricultural production due to lack of an ex-post crop assessment survey.

- $\quad$ Lack of a comprehensive economy-wide enterprise survey precludes reliable estimation of GDP from the production side. Instead, weak proxy indicators such as VAT data are used to estimate production.

- Quarterly national accounts are not benchmarked against annual survey, which limits usefulness of both quarterly and annual data due to lack of reliable annual surveys.

- $\quad$ The non-monetized informal sector activities - as estimated by 5-yearly household surveys - are assumed to grow at the population growth rate, giving a positive bias to GDP estimates.

\section{G. Program Monitoring}

\section{The first year of the program under the PSI will be monitored through} quantitative and structural conditionality (MEFP, Tables 2 and 3). The quantitative assessment criteria and indicative targets have been streamlined from a total of 13 in the previous PRGF arrangement, to 9 in the PSI, including a new indicative target (floor) on domestic revenue collection, given its importance in meeting one of the pillars of the PSI. Structural conditionalities, totaling eleven, cover the following areas: public financial management, revenue administration, financial sector, monetary policy, exchange rate, export sector, and statistics, with reforms that address the four key pillars of the PSI. Over the medium-term, structural conditionalities will continue to focus on reforms in PFM, revenue administration, and exchange rate management. The assessment dates for the first year are end-June 2010 and end-December 2010, each followed by reviews; indicative targets will be assessed at end-September 2010 and end-March 2011. 


\section{Staff Appraisal}

\section{Rwanda has made significant progress under the three successive PRGF}

arrangements, but challenges remain. Over the last decade, the country has achieved high growth and macroeconomic stability. Inflation, though volatile, is now in single digits, and international reserves are at comfortable levels. Rwanda's resiliency to external shocks has improved further as external debt has been reduced significantly - thanks to prudent policies and substantial debt relief - and heavy reliance on concessional borrowing has mitigated debt vulnerabilities. Rwanda's track record of prudent macroeconomic policies justifies transition to PSI-based Fund support.

28. The authorities are determined to address policy challenges under the new PSI. The program would support implementation of Rwanda's poverty strategy (the EDPRS), preserve macroeconomic stability and fiscal sustainability, strengthen monetary and exchange rate policies to deliver low and stable inflation, and implement growth-enhancing structural reforms aimed at deepening the financial sector, diversifying the export base, and improving the business environment.

\section{The authorities are committed to implementing significant reforms in all policy}

areas. Fiscal consolidation is frontloaded with revenue mobilization efforts underpinned by significant revenue administration reforms. The revenue targets are ambitious but achievable. Important revenue measures planned for the first half of 2010/11 are expected to have immediate impact on broadening the tax base for sustained higher revenues. Fiscal consolidation will also be sustained by the government's medium-term PFM strategy that is actively supported by the donor community. Low and stable inflation is anchored on the authorities' commitment to greater exchange rate flexibility — assisted by the MCM TA — as well as effective use of available monetary policy instruments, supported by an enhanced communication strategy and a more active interbank market. An ambitious structural reform agenda and an investment strategy address vulnerabilities arising from infrastructure bottlenecks, a narrow export base, and limited supervisory capacity in the financial sector.

\section{Vigilance is needed to mitigate the risks to the program, which appear}

manageable. Near term risks to the outlook include slower-than-expected pick up in external demand and in domestic credit conditions. Close monitoring of revenue administration reforms is needed to ensure their timely implementation. Efforts are also needed to ensure that priority spending is protected in case of revenue shortfalls. In the area of exchange rate policy, active implementation of the planned TA would ensure proper implementation of the exchange rate corridor would be a stepping stone towards greater exchange rate flexibility.

\section{The benefits and costs of the authorities' ambitious investment program must be} carefully assessed. The planned investments could generate employment, reduce poverty, and diversify the sources of growth thereby making the economy less susceptible to shocks. Moreover, the authorities intend to protect priority spending through their efforts to attract 
foreign investment. The risks, nevertheless, are non-negligible; they center on financing the investments, including direct lending, granting of generous tax incentives, and providing government loan guarantees on non concessional borrowing, including foreign currencylinked swaps. It is imperative to carefully assess the economic viability of the planned investment projects, sequence them properly, and consider financing options that crowd in private sector participation and limit fiscal risks.

32. Staff supports the authorities' request for the PSI. The PSI-supported program will help maintain macroeconomic stability while donor support appears to be strong. 
Table 1. Rwanda: Selected Economic and Financial Indicators, 2006-15

\begin{tabular}{|c|c|c|c|c|c|c|c|c|c|c|}
\hline & 2006 & 2007 & 2008 & 2009 & 2010 & 2011 & 2012 & 2013 & 2014 & 2015 \\
\hline & & & & Est. & \multicolumn{6}{|c|}{ Projection } \\
\hline & \multicolumn{10}{|c|}{ (Annual percentage changes, unless otherwise indicated) } \\
\hline \multicolumn{11}{|c|}{ ( } \\
\hline Real GDP growth & 9.2 & 5.5 & 11.2 & 4.1 & 5.4 & 5.9 & 6.4 & 6.9 & 6.5 & 6.5 \\
\hline Real GDP (per capita) & 7.3 & 3.3 & 8.9 & 2.0 & 3.2 & 3.7 & 4.2 & 4.7 & 4.3 & 4.4 \\
\hline GDP deflator & 9.8 & 13.2 & 12.6 & 11.0 & 6.7 & 6.1 & 5.6 & 5.0 & 5.0 & 5.0 \\
\hline Consumer prices (period average) & 8.8 & 9.1 & 15.4 & 10.4 & 6.4 & 6.5 & 5.5 & 5.0 & 5.0 & 5.0 \\
\hline Consumer prices (end of period) & 12.1 & 6.6 & 22.3 & 5.7 & 7.0 & 6.0 & 5.0 & 5.0 & 5.0 & 5.0 \\
\hline \multicolumn{11}{|l|}{ External sector } \\
\hline Export of goods, f.o.b (in U.S. dollars) & 17.9 & 19.9 & 51.4 & -28.0 & 24.6 & 15.3 & 13.4 & 14.8 & 15.6 & 17.1 \\
\hline Imports of goods, f.o.b (in U.S. dollars) & 26.2 & 30.2 & 51.5 & 9.3 & 19.1 & 12.8 & -4.8 & 3.2 & 6.2 & 7.8 \\
\hline Export volume & 12.5 & -0.1 & 21.8 & -20.0 & 11.0 & 13.1 & 13.6 & 15.7 & 17.4 & 14.8 \\
\hline Import volume & 27.0 & 31.0 & 20.2 & 9.4 & 9.1 & 7.5 & -6.7 & 1.6 & 6.9 & 6.8 \\
\hline Terms of trade (deterioration $=-$ ) & 5.5 & 20.9 & -1.4 & -9.9 & 2.9 & -2.8 & -2.2 & -2.3 & -0.9 & 1.1 \\
\hline \multicolumn{11}{|l|}{ Money and credit ${ }^{1}$} \\
\hline Net domestic assets ${ }^{2}$ & 2.6 & 7.6 & 10.3 & 4.9 & 10.9 & 17.0 & 12.2 & 12.1 & 10.0 & 9.8 \\
\hline Domestic credit $^{2}$ & 8.2 & 12.0 & 20.5 & 3.8 & 11.0 & 11.4 & 13.7 & 12.6 & 10.5 & 10.2 \\
\hline Government $^{2}$ & -5.6 & 0.2 & -18.1 & -0.4 & -2.5 & 0.0 & 0.0 & 0.0 & 0.0 & 0.0 \\
\hline Economy $^{2}$ & 13.8 & 11.9 & 38.6 & 4.2 & 13.5 & 11.4 & 13.7 & 12.6 & 10.5 & 10.2 \\
\hline Broad money (M2) & 31.3 & 30.8 & 24.2 & 13.1 & 12.4 & 12.3 & 12.3 & 12.3 & 12.3 & 12.3 \\
\hline Reserve money ${ }^{3}$ & 11.9 & 30.7 & 23.5 & 0.3 & 12.5 & 12.3 & 12.3 & 12.3 & 12.3 & 12.3 \\
\hline \multirow[t]{2}{*}{ Velocity (GDP/M2; end of period) } & 6.0 & 5.5 & 6.5 & 5.6 & 5.6 & 5.6 & 5.6 & 5.6 & 5.6 & 5.6 \\
\hline & \multicolumn{10}{|c|}{ (Percent of GDP) } \\
\hline \multicolumn{11}{|l|}{ National income accounts } \\
\hline National savings & 7.4 & 8.3 & 9.1 & 4.0 & 3.5 & 4.1 & 6.6 & 7.8 & 8.9 & 9.7 \\
\hline Gross investment & 19.7 & 20.2 & 23.5 & 21.3 & 22.8 & 22.7 & 21.5 & 20.8 & 20.4 & 20.3 \\
\hline Of which: private (including public enterprises) & 12.8 & 12.4 & 13.1 & 11.3 & 11.7 & 12.0 & 12.1 & 12.2 & 12.3 & 12.6 \\
\hline \multicolumn{11}{|l|}{ Government finance $^{4}$} \\
\hline Total revenue (excl. grants) & 12.2 & 12.4 & 12.6 & 14.9 & 12.2 & 13.6 & 13.6 & 14.2 & 14.5 & 14.7 \\
\hline Total expenditure and net lending & 23.7 & 22.0 & 22.6 & 26.4 & 25.9 & 26.9 & 25.9 & 24.9 & 24.0 & 23.3 \\
\hline Capital expenditure & 7.6 & 7.0 & 8.2 & 11.1 & 9.8 & 11.4 & 10.2 & 9.0 & 8.3 & 7.9 \\
\hline Current expenditure & 15.9 & 14.2 & 15.1 & 14.5 & 14.9 & 14.6 & 15.2 & 15.4 & 15.3 & 15.3 \\
\hline Primary fiscal balance ${ }^{5}$ & -2.9 & -1.2 & -2.4 & 1.1 & -3.2 & -1.1 & -2.2 & -1.6 & -0.8 & -0.4 \\
\hline Domestic fiscal balance $^{6}$ & -6.1 & -5.4 & -7.3 & -7.7 & -13.4 & -13.6 & -13.1 & -12.8 & -11.9 & -11.4 \\
\hline \multicolumn{11}{|l|}{ Overall fiscal balance (payment order) } \\
\hline After grants & 5.2 & -1.4 & -0.2 & -2.2 & -1.1 & -3.6 & -1.5 & -0.6 & -0.4 & -0.4 \\
\hline Before grants & -11.5 & -9.6 & -10.0 & -11.5 & -13.7 & -13.4 & -12.4 & -10.7 & -9.4 & -8.6 \\
\hline \multicolumn{11}{|l|}{ External sector } \\
\hline \multicolumn{11}{|l|}{ External current account balance } \\
\hline Including official transfers & -4.3 & -2.2 & -4.9 & -7.3 & -7.9 & -8.8 & -5.4 & -4.4 & -3.7 & -3.5 \\
\hline Excluding official transfers & -12.3 & -11.9 & -14.4 & -17.3 & -19.3 & -18.6 & -14.8 & -12.9 & -11.6 & -10.6 \\
\hline External debt (end of period) & 15.6 & 15.3 & 14.4 & 14.4 & 16.4 & 19.3 & 19.1 & 18.0 & 16.7 & 15.4 \\
\hline \multicolumn{11}{|l|}{ Net present value of external debt } \\
\hline (percent of exports of goods and services) & 53.1 & 57.7 & 49.9 & 89.0 & 102.8 & 125.2 & 120.6 & 107.4 & 94.2 & 82.2 \\
\hline \multicolumn{11}{|l|}{ Scheduled debt service ratio } \\
\hline \multirow[t]{2}{*}{ Gross reserves (in months of imports of goods and services) ${ }^{7}$} & 5.6 & 4.7 & 4.8 & 5.1 & 4.8 & 4.7 & 4.7 & 4.4 & 4.3 & 4.2 \\
\hline & & & & (Millions & f U.S. de & Ilars) & & & & \\
\hline Gross official reserves & 439.6 & 552.4 & 596.4 & 735.3 & 744.3 & 700.6 & 709.4 & 709.2 & 739.2 & 776.3 \\
\hline Memorandum item: & & & & & & & & & & \\
\hline Nominal GDP (billions of Rwanda francs) & 1,716 & 2,049 & 2,565 & 2,964 & 3,333 & 3,746 & 4,210 & 4,725 & 5,285 & 5,912 \\
\hline Sources: Rw andan authorities; and IMF staff estimates and projections. & & & & & & & & & & \\
\hline${ }^{1}$ Projections & 571.24 . & & & & & & & & & \\
\hline${ }^{2}$ As a percent of the beginning-of-period stock of broad money. & & & & & & & & & & \\
\hline${ }^{3}$ Increase in 2007 reflects rebasing of the monetary program; reserve mon & ow th $w$ as limite & 13 perce & fter corre & for the ret & ing at en & -2006. & & & & \\
\hline${ }^{4}$ On a fiscal year basis (Julky-June).For example, the column ending in 20 & fer to FY2010/ & & & & & & & & & \\
\hline $\begin{array}{l}{ }^{5} \text { Revenue excluding grants minus current expenditure except interest due } \\
\text { minus domestically financed capital expenditure. }\end{array}$ & xceptional exp & ture (AU & cekeepin & enditures & spendin & on den & lizing a & ntegra & militia & \\
\hline
\end{tabular}


Table 2. Rwanda: Operations of the Central Government, Fiscal Year Basis ${ }^{1}, 2006 / 07-14 / 15$

\begin{tabular}{|c|c|c|c|c|c|c|c|c|c|}
\hline & $2006 / 07$ & $2007 / 08$ & $2008 / 09$ & $2009 / 10$ & $2010 / 11$ & $2011 / 12$ & $2012 / 13$ & $2013 / 14$ & $2014 / 15$ \\
\hline & & & & Proj. & Proj. & Proj. & Proj. & Proj. & Proj. \\
\hline & \multicolumn{9}{|c|}{ (Billions of Rwandan francs) } \\
\hline Revenue and grants & 388.0 & 518.2 & 670.7 & 779.6 & 826.0 & 970.7 & $1,083.2$ & $1,178.7$ & $1,286.7$ \\
\hline Total revenue & 233.2 & 290.3 & 413.0 & 385.1 & 479.7 & 539.7 & 632.7 & 727.3 & 824.5 \\
\hline Tax revenue & 217.6 & 272.4 & 361.4 & 369.3 & 457.6 & 512.6 & 597.6 & 688.0 & 780.5 \\
\hline Direct taxes & 73.6 & 103.3 & 130.1 & 144.5 & 178.9 & 201.4 & 230.8 & 268.6 & 306.0 \\
\hline Taxes on goods and services & 109.8 & 135.0 & 179.3 & 191.2 & 237.7 & 265.4 & 315.8 & 363.8 & 412.5 \\
\hline Taxes on international trade & 34.2 & 34.2 & 52.0 & 33.6 & 41.0 & 45.8 & 51.0 & 55.6 & 62.0 \\
\hline Nontax revenue & 15.7 & 17.8 & 51.7 & 15.8 & 22.1 & 27.1 & 35.1 & 39.3 & 44.0 \\
\hline ID receipts & $\ldots$ & $\ldots$ & 3.2 & 1.5 & $\ldots$ & $\ldots$ & $\ldots$ & $\ldots$ & $\ldots$ \\
\hline Grants & 154.8 & 227.9 & 257.7 & 394.5 & 346.3 & 431.0 & 450.5 & 451.4 & 462.2 \\
\hline Budgetary grants & 101.4 & 168.1 & 167.0 & 268.1 & 229.5 & 317.3 & 333.0 & 332.0 & 340.8 \\
\hline Of which: HIPC Initiative assistance & 7.2 & 1.5 & 2.4 & 3.0 & $1.8^{4}$ & & $\ldots$ & $\ldots$ & \\
\hline Capital grants & 53.4 & 59.8 & 90.7 & 126.4 & 116.8 & 113.7 & 117.5 & 119.4 & 121.4 \\
\hline Project grants & 53.4 & 59.8 & 90.7 & 126.4 & 116.8 & 113.7 & 117.5 & 119.4 & 121.4 \\
\hline Total expenditure and net lending & 414.2 & 521.8 & 731.2 & 814.9 & 952.6 & $1,031.8$ & $1,111.9$ & $1,199.0$ & $1,306.8$ \\
\hline Current expenditure & 266.5 & 349.0 & 401.7 & 467.7 & 517.4 & 606.5 & 689.3 & 766.9 & 854.5 \\
\hline Of which: priority & 139.7 & 151.7 & 232.2 & 280.4 & 297.6 & $\ldots$ & $\ldots$ & $\ldots$ & \\
\hline Wages and salaries & 71.5 & 76.3 & 90.8 & 108.2 & 120.4 & 130.1 & 146.4 & 164.9 & 184.5 \\
\hline Civil & 48.5 & 55.5 & 66.1 & 78.9 & 85.7 & 94.0 & 105.8 & 116.9 & 130.8 \\
\hline Defense & 22.9 & 20.8 & 24.7 & 29.3 & 34.7 & 36.1 & 40.6 & 48.0 & 53.7 \\
\hline Purchases of goods and services & 66.4 & 81.6 & 103.2 & 106.8 & 121.0 & 156.7 & 180.8 & 203.6 & 227.1 \\
\hline Civil & 57.5 & 70.4 & 82.9 & 94.5 & 105.3 & 135.3 & 156.9 & 174.7 & 195.1 \\
\hline Defense & 8.9 & 11.2 & 20.2 & 12.3 & 15.7 & 21.4 & 23.9 & 28.9 & 32.0 \\
\hline Interest payments & 12.8 & 13.6 & 11.4 & 13.4 & 13.5 & 14.4 & 15.8 & 16.2 & 18.4 \\
\hline Domestic debt & 8.7 & 11.1 & 7.4 & 8.6 & 8.9 & 9.3 & 9.7 & 10.2 & 11.4 \\
\hline External debt & 4.1 & 2.4 & 4.0 & 4.8 & 4.6 & 5.1 & 6.1 & 6.0 & 7.0 \\
\hline Transfers & 82.4 & 121.8 & 141.6 & 184.7 & 195.5 & 237.2 & 279.2 & 313.1 & 347.3 \\
\hline Exceptional expenditure & 33.5 & 55.7 & 54.7 & 54.7 & 67.0 & 68.1 & 67.1 & 69.1 & 77.3 \\
\hline Capital expenditure & 131.8 & 188.2 & 306.6 & 308.8 & 404.0 & 404.0 & 400.3 & 417.2 & 442.6 \\
\hline Of which: priority & 28.0 & 38.9 & 124.4 & 129.2 & 158.9 & $\ldots$ & $\ldots$ & $\ldots$ & \\
\hline Domestic & 42.5 & 87.6 & 139.7 & 152.4 & 224.3 & 210.9 & 233.7 & 256.0 & 278.7 \\
\hline Foreign & 89.3 & 100.6 & 167.0 & 156.4 & 179.7 & 193.2 & 166.6 & 161.2 & 163.9 \\
\hline $\begin{array}{l}\text { Net lending and privatization receipts } \\
\text { of which: }\end{array}$ & 15.9 & -15.4 & 22.9 & 38.4 & 31.2 & 21.3 & 22.3 & 14.9 & 9.7 \\
\hline Priority spending & 4.8 & 3.9 & 2.1 & 2.9 & 3.6 & & $\ldots$ & $\ldots$ & \\
\hline Kigali Convention Center & 0.0 & 0.0 & 0.0 & 18.0 & 0.0 & 0.0 & 0.0 & 0.0 & 0.0 \\
\hline RwandAir & 0.0 & 0.0 & 0.0 & 12.0 & 25.0 & 11.7 & 7.1 & 0.0 & 0.0 \\
\hline Primary balance ${ }^{2}$ & -29.5 & -77.1 & -62.2 & -167.0 & -24.2 & -52.4 & -40.8 & -23.4 & -11.6 \\
\hline Domestic fiscal balance ${ }^{3}$ & -87.5 & -128.5 & -147.2 & -268.7 & -288.7 & -293.9 & -306.5 & -304.4 & -311.5 \\
\hline Excluding demobilization and peacekeeping expenditures & -76.6 & -112.0 & -121.8 & -245.1 & -252.3 & -278.4 & -306.5 & -304.4 & -311.5 \\
\hline \multicolumn{10}{|l|}{ Overall deficit (payment order) } \\
\hline After grants & -26.1 & -3.6 & -60.5 & -35.4 & -126.6 & -61.1 & -28.7 & -20.2 & -20.1 \\
\hline Before grants & -180.9 & -231.6 & -318.1 & -429.9 & -472.9 & -492.1 & -479.2 & -471.6 & -482.3 \\
\hline Change in arrears ${ }^{4}$ & -7.0 & -8.6 & -9.0 & -8.4 & -8.0 & -8.0 & -8.0 & -8.0 & -8.0 \\
\hline Overall deficit (incl. grants, cash basis) & -33.1 & -12.2 & -69.4 & -43.8 & -134.6 & -69.1 & -36.7 & -28.2 & -28.1 \\
\hline Financing & 41.2 & 11.3 & 69.4 & 43.8 & 134.6 & 69.1 & 36.7 & 28.2 & 28.1 \\
\hline Foreign financing (net) & 39.2 & 42.8 & 72.5 & 24.7 & 54.6 & 69.2 & 36.7 & 28.2 & 28.1 \\
\hline Drawings & 44.7 & 47.9 & 77.0 & 30.0 & 62.9 & 79.5 & 49.1 & 41.8 & 42.5 \\
\hline Budgetary loans & 8.8 & 5.2 & 0.7 & 0.0 & 0.0 & 0.0 & 0.0 & 0.0 & 0.0 \\
\hline Project loans & 36.0 & 42.7 & 76.3 & 30.0 & 62.9 & 79.5 & 49.1 & 41.8 & 42.5 \\
\hline Amortization & -5.6 & -5.1 & -4.4 & -5.3 & -8.3 & -10.3 & -12.4 & -13.6 & -14.4 \\
\hline Net domestic financing & 2.1 & -31.5 & -3.1 & 19.0 & 80.1 & 0.0 & 0.0 & 0.0 & 0.0 \\
\hline Net credit from banking system & -7.2 & -13.4 & 18.0 & 20.8 & 51.3 & 0.0 & 0.0 & 0.0 & 0.0 \\
\hline Nonbank sector & 9.3 & -18.1 & -21.2 & -1.8 & 28.8 & 0.0 & 0.0 & 0.0 & 0.0 \\
\hline Errors and omissions / Financing gap 5 & 8.1 & -0.9 & 0.0 & 0.0 & 0.0 & 0.0 & 0.0 & 0.0 & 0.0 \\
\hline \multicolumn{10}{|l|}{ Memo: } \\
\hline Priority spending & 172.5 & 194.5 & 358.7 & 412.5 & 460.1 & $\ldots$ & $\ldots$ & $\ldots$ & $\ldots$ \\
\hline
\end{tabular}

Sources: Rwandan authorities, and IMF staff estimates and projections.

${ }^{1}$ Fiscal year runs from July to June.

2 Total revenue minus noninterest current expenditure (excluding exceptional expenditure) minus domestically financed capital investment.

${ }^{3}$ Total revenue minus current expenditure (excluding interest ion external debt), domestically financed capital expenditure, and net lending

${ }^{4}$ A negative sign indicates a reduction.

${ }^{5}$ A negative number implies an underestimate of financing. 
Table 2. Rwanda: Operations of the Central Government, Fiscal Year Basis ${ }^{1}, 2006 / 07-14 / 15$ (continued)

\begin{tabular}{|c|c|c|c|c|c|c|c|c|c|}
\hline & $2006 / 07$ & $2007 / 08$ & $2008 / 09$ & $\begin{array}{r}\text { 2009/10 } \\
\text { Proj. }\end{array}$ & $\begin{array}{r}\text { 2010/11 } \\
\text { Proj. }\end{array}$ & $\begin{array}{r}2011 / 12 \\
\text { Proj. }\end{array}$ & $\begin{array}{r}\text { 2012/13 } \\
\text { Proj. }\end{array}$ & $\begin{array}{r}2013 / 14 \\
\text { Proj. }\end{array}$ & $\begin{array}{r}2014 / 15 \\
\text { Proj. }\end{array}$ \\
\hline & \multicolumn{9}{|c|}{ (In percent of fiscal year GDP) } \\
\hline Revenue and grants & 20.6 & 22.5 & 24.3 & 24.8 & 23.3 & 24.4 & 24.2 & 23.6 & 23.0 \\
\hline Total revenue & 12.4 & 12.6 & 14.9 & 12.2 & 13.6 & 13.6 & 14.2 & 14.5 & 14.7 \\
\hline Tax revenue & 11.6 & 11.8 & 13.1 & 11.7 & 12.9 & 12.9 & 13.4 & 13.7 & 13.9 \\
\hline Direct taxes & 3.9 & 4.5 & 4.7 & 4.6 & 5.1 & 5.1 & 5.2 & 5.4 & 5.5 \\
\hline Taxes on goods and services & 5.8 & 5.8 & 6.5 & 6.1 & 6.7 & 6.7 & 7.1 & 7.3 & 7.4 \\
\hline Taxes on international trade & 1.8 & 1.5 & 1.9 & 1.1 & 1.2 & 1.2 & 1.1 & 1.1 & 1.1 \\
\hline Nontax revenue & 0.8 & 0.8 & 1.9 & 0.5 & 0.6 & 0.7 & 0.8 & 0.8 & 0.8 \\
\hline ID receipts & $\ldots$ & $\ldots$ & 0.1 & 0.0 & $\ldots$ & $\ldots$ & $\ldots$ & $\ldots$ & $\ldots$ \\
\hline Grants & 8.2 & 9.9 & 9.3 & 12.5 & 9.8 & 10.8 & 10.1 & 9.0 & 8.3 \\
\hline Budgetary grants & 5.4 & 7.3 & 6.0 & 8.5 & 6.5 & 8.0 & 7.5 & 6.6 & 6.1 \\
\hline Of which: HIPC Initiative assistance & 0.4 & 0.1 & 0.1 & 0.1 & 0.1 & & $\ldots$ & $\ldots$ & \\
\hline Capital grants & 2.8 & 2.6 & 3.3 & 4.0 & 3.3 & 2.9 & 2.6 & 2.4 & 2.2 \\
\hline Project grants & 2.8 & 2.6 & 3.3 & 4.0 & 3.3 & 2.9 & 2.6 & 2.4 & 2.2 \\
\hline Total expenditure and net lending & 22.0 & 22.6 & 26.4 & 25.9 & 26.9 & 25.9 & 24.9 & 24.0 & 23.3 \\
\hline Current expenditure & 14.2 & 15.1 & 14.5 & 14.9 & 14.6 & 15.2 & 15.4 & 15.3 & 15.3 \\
\hline Of which: priority & 7.4 & 6.6 & 8.4 & 8.9 & 8.4 & $\ldots$ & $\ldots$ & $\ldots$ & $\ldots$ \\
\hline Wages and salaries & 3.8 & 3.3 & 3.3 & 3.4 & 3.4 & 3.3 & 3.3 & 3.3 & 3.3 \\
\hline Civil & 2.6 & 2.4 & 2.4 & 2.5 & 2.4 & 2.4 & 2.4 & 2.3 & 2.3 \\
\hline Defense & 1.2 & 0.9 & 0.9 & 0.9 & 1.0 & 0.9 & 0.9 & 1.0 & 1.0 \\
\hline Purchases of goods and services & 3.5 & 3.5 & 3.7 & 3.4 & 3.4 & 3.9 & 4.0 & 4.1 & 4.1 \\
\hline Civil & 3.1 & 3.0 & 3.0 & 3.0 & 3.0 & 3.4 & 3.5 & 3.5 & 3.5 \\
\hline Defense & 0.5 & 0.5 & 0.7 & 0.4 & 0.4 & 0.5 & 0.5 & 0.6 & 0.6 \\
\hline Interest payments & 0.7 & 0.6 & 0.4 & 0.4 & 0.4 & 0.4 & 0.4 & 0.3 & 0.3 \\
\hline Domestic debt & 0.5 & 0.5 & 0.3 & 0.3 & 0.3 & 0.2 & 0.2 & 0.2 & 0.2 \\
\hline External debt & 0.2 & 0.1 & 0.1 & 0.2 & 0.1 & 0.1 & 0.1 & 0.1 & 0.1 \\
\hline Transfers & 4.4 & 5.3 & 5.1 & 5.9 & 5.5 & 6.0 & 6.2 & 6.3 & 6.2 \\
\hline Exceptional expenditure & 1.8 & 2.4 & 2.0 & 1.7 & 1.9 & 1.7 & 1.5 & 1.4 & 1.4 \\
\hline Capital expenditure & 7.0 & 8.2 & 11.1 & 9.8 & 11.4 & 10.2 & 9.0 & 8.3 & 7.9 \\
\hline Of which: priority & 1.5 & 1.7 & 4.5 & 4.1 & 4.5 & & $\ldots$ & $\ldots$ & \\
\hline Domestic & 2.3 & 3.8 & 5.1 & 4.8 & 6.3 & 5.3 & 5.2 & 5.1 & 5.0 \\
\hline Foreign & 4.7 & 4.4 & 6.0 & 5.0 & 5.1 & 4.9 & 3.7 & 3.2 & 2.9 \\
\hline Net lending and privatization receipts & 0.8 & -0.7 & 0.8 & 1.2 & 0.9 & 0.5 & 0.5 & 0.3 & 0.2 \\
\hline of which: & 0.0 & 0.0 & 0.0 & 0.0 & 0.0 & 0.0 & 0.0 & 0.0 & 0.0 \\
\hline Priority spending & 0.3 & 0.2 & 0.1 & 0.1 & 0.1 & $\ldots$ & $\ldots$ & $\ldots$ & $\ldots$ \\
\hline Kigali Convention Center & 0.0 & 0.0 & 0.0 & 0.6 & 0.0 & 0.0 & 0.0 & 0.0 & 0.0 \\
\hline RwandAir & 0.0 & 0.0 & 0.0 & 0.4 & 0.7 & 0.3 & 0.2 & 0.0 & 0.0 \\
\hline Primary balance ${ }^{2}$ & -1.6 & -3.3 & -2.2 & -5.3 & -0.7 & -1.3 & -0.9 & -0.5 & -0.2 \\
\hline Domestic fiscal balance $^{3}$ & -4.6 & -5.6 & -5.3 & -8.5 & -8.2 & -7.4 & -6.9 & -6.1 & -5.6 \\
\hline Excluding demobilization and peacekeeping expenditures & -4.1 & -4.9 & -4.4 & -7.8 & -7.1 & -7.0 & -6.9 & -6.1 & -5.6 \\
\hline \multicolumn{10}{|l|}{ Overall deficit (payment order) } \\
\hline After grants & -1.4 & -0.2 & -2.2 & -1.1 & -3.6 & -1.5 & -0.6 & -0.4 & -0.4 \\
\hline \multirow[t]{2}{*}{ Before grants } & -9.6 & -10.0 & -11.5 & -13.7 & -13.4 & -12.4 & -10.7 & -9.4 & -8.6 \\
\hline & 0.0 & 0.0 & 0.0 & 0.0 & 0.0 & 0.0 & 0.0 & 0.0 & 0.0 \\
\hline Change in arrears ${ }^{4}$ & -0.4 & -0.4 & -0.3 & -0.3 & -0.2 & -0.2 & -0.2 & -0.2 & -0.1 \\
\hline Overall deficit (incl. grants, cash basis) & -1.8 & -0.5 & -2.5 & -1.4 & -3.8 & -1.7 & -0.8 & -0.6 & -0.5 \\
\hline Financing & 2.2 & 0.5 & 2.5 & 1.4 & 3.8 & 1.7 & 0.8 & 0.6 & 0.5 \\
\hline Foreign financing (net) & 2.1 & 1.9 & 2.6 & 0.8 & 1.5 & 1.7 & 0.8 & 0.6 & 0.5 \\
\hline Drawings & 2.4 & 2.1 & 2.8 & 1.0 & 1.8 & 2.0 & 1.1 & 0.8 & 0.8 \\
\hline Budgetary loans & 0.5 & 0.2 & 0.0 & 0.0 & 0.0 & 0.0 & 0.0 & 0.0 & 0.0 \\
\hline Project loans & 1.9 & 1.9 & 2.8 & 1.0 & 1.8 & 2.0 & 1.1 & 0.8 & 0.8 \\
\hline Amortization & -0.3 & -0.2 & -0.2 & -0.2 & -0.2 & -0.3 & -0.3 & -0.3 & -0.3 \\
\hline Net domestic financing & 0.1 & -1.4 & -0.1 & 0.6 & 2.3 & 0.0 & 0.0 & 0.0 & 0.0 \\
\hline Net credit from banking system & -0.4 & -0.6 & 0.7 & 0.7 & 1.4 & 0.0 & 0.0 & 0.0 & 0.0 \\
\hline Nonbank sector & 0.5 & -0.8 & -0.8 & -0.1 & 0.8 & 0.0 & 0.0 & 0.0 & 0.0 \\
\hline Errors and omissions / Financing gap 5 & 0.4 & 0.0 & 0.0 & 0.0 & 0.0 & 0.0 & 0.0 & 0.0 & 0.0 \\
\hline \multicolumn{10}{|l|}{ Memo: } \\
\hline Priority spending & 9.2 & 8.4 & 13.0 & 13.1 & 13.0 & $\ldots$ & $\ldots$ & $\ldots$ & \\
\hline GDP (Billions of RwF), FY basis & 1,883 & 2,307 & 2,765 & 3,149 & 3,540 & 3,978 & 4,468 & 5,005 & 5,599 \\
\hline
\end{tabular}

Sources: Rwandan authorities, and IMF staff estimates and projections.

1 Fiscal year runs from July to June.

2 Total revenue minus noninterest current expenditure (excluding exceptional expenditure) minus domestically financed capital investment.

${ }^{3}$ Total revenue minus current expenditure (excluding interest on external debt), domestically financed capital expenditure, and net lending

${ }^{4}$ A negative sign indicates a reduction.

${ }^{5}$ A negative number implies an underestimate of financing. 
Table 3. Rwanda: Monetary Survey ${ }^{1}$, 2006-15

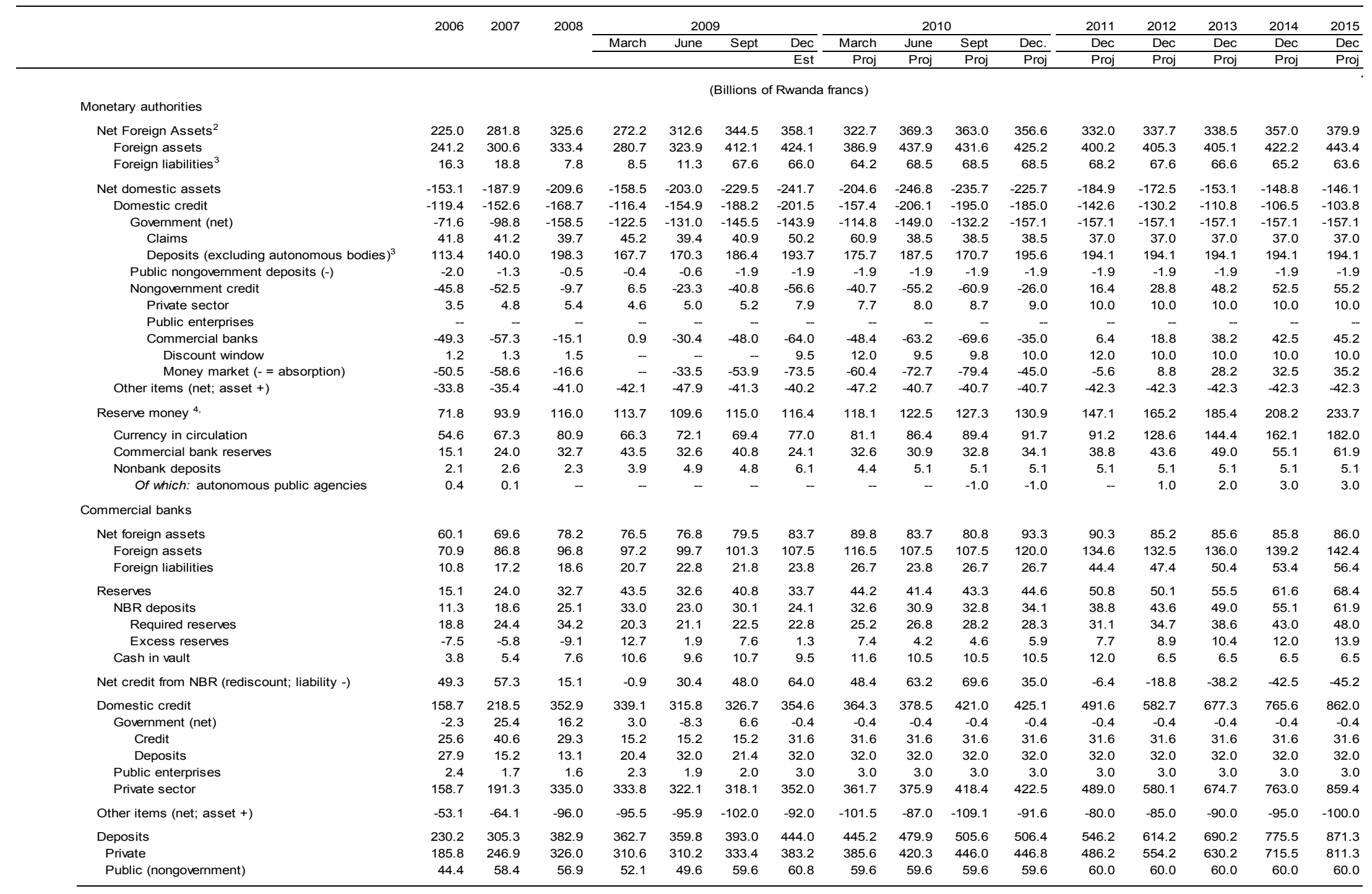


Table 3. Rwanda: Monetary Survey, 2006-15 (concluded)

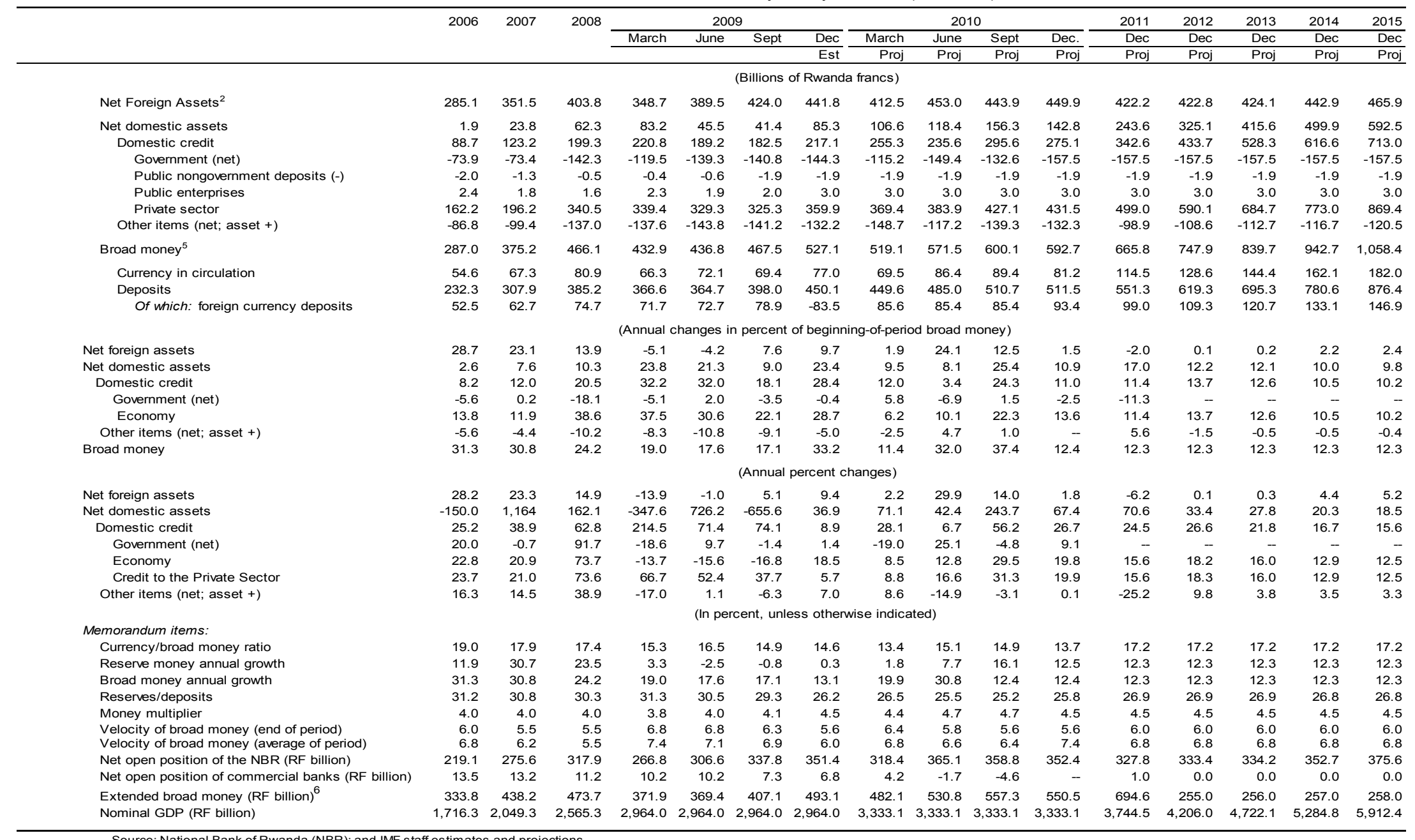
IMF staff estimates and projections.

1 Reflects the operations of the Union Banques Populaires du Rwanda (UBPR)—a large microfinance network - which was transformed into a commercial bank in Febuary 2008.

2 For program purposes NFA from 2010 onwards are at program exchange rates.

${ }^{3}$ The IMF'S MDRI reduced foreign liabilities at the NBR by RF 42.4 million with a counter entry in government deposits (in January 2006).

${ }^{4}$ Reserve money as an Assessment Criteria is measured as the average of the months in the quarter. The actual rerserve money is measured as the daily average of the last month in the quarter.

${ }^{5}$ End-2006 broad money includes RF5 billion temporary build up of local government deposits, which were unwound by February 2007.

${ }^{6}$ Broad money plus deposits in the Union de Banques Populaires de Rwanda (UBPR) through December 2008 and Rwanda Development Bank (BRD). 
Table 4. Rwanda: Financial Soundness Indicators for Banking Sector, 2006-2009

(in percent)

\begin{tabular}{|c|c|c|c|c|c|c|c|c|c|}
\hline & $\begin{array}{l}2006 \\
\text { Dec }\end{array}$ & $\begin{array}{l}2007 \\
\text { Dec }\end{array}$ & $\begin{array}{c}2008 \\
\text { Mar }\end{array}$ & $\begin{array}{l}2008 \\
\text { June }\end{array}$ & $\begin{array}{l}2008 \\
\text { Dec }\end{array}$ & $\begin{array}{c}2009 \\
\text { Mar }\end{array}$ & $\begin{array}{l}2009 \\
\text { June }\end{array}$ & $\begin{array}{c}2009 \\
\text { Sep }\end{array}$ & $\begin{array}{c}2009 \\
\text { Dec }\end{array}$ \\
\hline \multicolumn{10}{|l|}{ Capital adequacy } \\
\hline Solvency ratio & 13.7 & 16.6 & 15.1 & 18.0 & 15.9 & 19.1 & 19.3 & 20.5 & 19.0 \\
\hline Off balance sheet items/Total qualifying capital & 334.8 & 305.6 & 221.7 & 205.9 & 210.2 & 182.5 & 160.7 & 164.0 & 184.1 \\
\hline Insider loans/Core capital & 45.2 & 57.9 & 20.1 & 9.3 & 16.7 & 9.2 & 17.0 & 7.3 & 19.7 \\
\hline Large exposure/core capital & 131.6 & 127.7 & 77.9 & 67.5 & 103.1 & 92.7 & 82.8 & 72.9 & 65.1 \\
\hline \multicolumn{10}{|l|}{ Asset quality } \\
\hline NPLs / Gross Loans & 25.0 & 18.1 & 12.2 & 12.0 & 12.6 & 13.8 & 13.4 & 13.6 & 13.1 \\
\hline NPLS net/Net loans & 12.5 & 14.3 & 9.6 & 8.0 & 10.5 & 11.0 & 11.1 & 11.7 & 11.4 \\
\hline Provisions / NPLs & 83.5 & 67.0 & 78.1 & 75.5 & 66.3 & 67.6 & 70.9 & 65.9 & 55.2 \\
\hline Earning Assets / Total Asset & 85.3 & 59.6 & 80.1 & 80.9 & 81.3 & 78.4 & 77.3 & 77.1 & 81.7 \\
\hline Large Exposures / Gross Loans & 20.2 & 21.2 & 12.6 & 13.0 & 17.4 & 18.4 & 17.5 & 16.9 & 13.9 \\
\hline \multicolumn{10}{|l|}{ Profitability and earnings } \\
\hline Return on Average Assets & 2.4 & 1.5 & 4.9 & 2.7 & 2.4 & 1.6 & 0.9 & 1.0 & 0.7 \\
\hline Return on Average Equity & 27.0 & 15.5 & 10.8 & 21.9 & 18.5 & 11.4 & 6.4 & 7.0 & 5.0 \\
\hline Net Interest Margin & 8.0 & 5.2 & 10.1 & 8.9 & 9.5 & 10.3 & 10.2 & 9.9 & 9.1 \\
\hline Cost of Deposits & 2.6 & 2.6 & 1.8 & 1.9 & 1.8 & 1.7 & 2.1 & 2.3 & 2.4 \\
\hline Cost to Income & 76.7 & 80.2 & 64.1 & 74.1 & 77.5 & 83.7 & 89.8 & 89.8 & 91.0 \\
\hline Overhead to income & 46.2 & 44.2 & 41.6 & 46.7 & 48.6 & 52.2 & 53.4 & 55.5 & 54.9 \\
\hline \multicolumn{10}{|l|}{ Liquidity } \\
\hline Short term Gap & 34.2 & 21.5 & 13.6 & 25.5 & 21.3 & 17.2 & 20.1 & 20.1 & 20.0 \\
\hline Liquid Assets / Total Deposits & 64.7 & 76.4 & 54.0 & 60.4 & 61.1 & 65.4 & 59.3 & 61.7 & 65.3 \\
\hline Interbank Borrowings / Total Deposits & 11.3 & 10.3 & 6.6 & 5.7 & 8.1 & 9.3 & 9.1 & 8.2 & 9.8 \\
\hline BNR Borrowings / Total Deposits & 0.3 & 0.2 & 0.2 & 0.1 & 0.2 & 0.1 & 0.1 & 0.4 & 0.8 \\
\hline Gross loans/Total deposits & 71.1 & 63.3 & 81.0 & 76.2 & 87.8 & 88.6 & 82.8 & 76.1 & 73.9 \\
\hline \multicolumn{10}{|l|}{ Market sensitivity } \\
\hline Forex Exposure / Core Capital ' & 9.0 & 8.7 & 2.6 & -5.2 & -4.6 & 0.6 & 5.9 & 0.8 & 1.9 \\
\hline Forex Loans / Forex Deposits & 0.8 & 1.0 & 1.2 & 0.5 & 0.5 & 0.4 & 0.6 & 0.5 & 2.8 \\
\hline Forex Assets / Forex Liabilities & 104.4 & 112.0 & 115.6 & 105.5 & 102.6 & 93.5 & 107.7 & 102.8 & 103.6 \\
\hline
\end{tabular}

Source: National Bank of Rw anda

${ }^{1}$ Data from 2007 and 2008 have been revised. 
Table 5. Rwanda: Balance of Payments, $2007-15$

(Millions of U.S. dollars, unless otherwise indicated)

\begin{tabular}{|c|c|c|c|c|c|c|c|c|c|}
\hline & 2007 & $\begin{array}{c}2008 \\
\text { Est. }\end{array}$ & $\begin{array}{r}2009 \\
\text { Est. }\end{array}$ & $\begin{array}{l}2010 \\
\text { Proj. }\end{array}$ & $\begin{array}{l}2011 \\
\text { Proj. }\end{array}$ & $\begin{array}{l}2012 \\
\text { Proj. }\end{array}$ & $\begin{array}{l}2013 \\
\text { Proj. }\end{array}$ & $\begin{array}{l}2014 \\
\text { Proj. }\end{array}$ & $\begin{array}{l}2015 \\
\text { Proj. }\end{array}$ \\
\hline Exports (f.o.b.), of which: & 176.8 & 267.7 & 192.7 & 240.1 & 276.9 & 314.1 & 360.7 & 416.9 & 488.3 \\
\hline Coffee and tea & 67.2 & 92.0 & 85.6 & 107.6 & 120.3 & 137.2 & 156.4 & 169.5 & 192.0 \\
\hline Minerals & 70.6 & 91.7 & 55.4 & 74.3 & 90.3 & 104.5 & 120.4 & 136.3 & 159.8 \\
\hline Imports (f.o.b.), of which: & 581.2 & 880.7 & 963.0 & $1,146.6$ & $1,293.5$ & $1,231.7$ & $1,271.4$ & $1,350.6$ & $1,456.2$ \\
\hline Capital goods & 152.7 & 275.5 & 272.1 & 351.2 & 439.6 & 316.3 & 293.3 & 315.1 & 347.5 \\
\hline Energy goods & 87.2 & 121.1 & 156.6 & 213.8 & 250.5 & 268.4 & 286.3 & 298.2 & 321.2 \\
\hline Trade balance & -404.4 & -613.0 & -770.3 & -906.4 & $-1,016.5$ & -917.6 & -910.7 & -933.8 & -968.0 \\
\hline Services (net) ${ }^{1}$ & -123.2 & -100.6 & -178.5 & -214.1 & -132.2 & -72.0 & -29.3 & 3.5 & 25.4 \\
\hline Of which: tourism receipts & 151.6 & 186.0 & 174.5 & 205.9 & 236.7 & 272.2 & 313.1 & 350.7 & 385.7 \\
\hline Income & -17.2 & -35.1 & -36.8 & -58.5 & -74.7 & -86.3 & -88.6 & -86.4 & -88.1 \\
\hline Of which: interest on public debt ${ }^{2}$ & -6.2 & -6.9 & -7.3 & -9.1 & -16.1 & -25.7 & -28.4 & -27.0 & -25.2 \\
\hline Current transfers (net) ${ }^{3}$ & 461.3 & 518.6 & 604.0 & 728.5 & 686.4 & 714.9 & 708.4 & 722.9 & 732.4 \\
\hline Private & 98.8 & 72.6 & 79.7 & 82.2 & 85.0 & 88.8 & 92.5 & 107.1 & 123.5 \\
\hline Of which: remittance inflows & 98.5 & 63.3 & 88.1 & 91.6 & 95.5 & 100.2 & 105.4 & 115.8 & 127.6 \\
\hline Public & 362.5 & 446.0 & 524.3 & 646.4 & 601.4 & 626.1 & 615.9 & 615.9 & 608.9 \\
\hline Of which: HIPC grants & 4.5 & 5.6 & 5.2 & 4.7 & 4.5 & 4.9 & 5.3 & 5.6 & 5.6 \\
\hline Current account balance (incl. official transfers) & -83.4 & -230.1 & -381.5 & -450.5 & -537.0 & -361.0 & -320.2 & -293.7 & -298.3 \\
\hline Current account balance (excl. official transfers) & -445.9 & -676.1 & -905.8 & $-1,096.9$ & $-1,138.5$ & -987.1 & -936.2 & -909.6 & -907.2 \\
\hline Capital account & 92.0 & 210.1 & 200.0 & 207.5 & 181.8 & 184.5 & 181.6 & 179.2 & 176.9 \\
\hline Project grants & 92.0 & 210.1 & 200.0 & 207.5 & 181.8 & 184.5 & 181.6 & 179.2 & 176.9 \\
\hline Financial account & 104.7 & 106.1 & 312.2 & 246.5 & 312.2 & 186.5 & 140.2 & 147.0 & 161.4 \\
\hline Direct investment & 82.3 & 103.4 & 118.7 & 102.6 & 82.8 & 106.9 & 127.4 & 142.3 & 163.6 \\
\hline Public sector capital & 80.4 & 104.8 & 169.0 & 161.9 & 243.0 & 89.6 & 32.4 & 13.8 & 6.9 \\
\hline Long-term borrowing ${ }^{4}$ & 88.7 & 112.2 & 75.7 & 176.4 & 275.3 & 123.4 & 78.9 & 66.6 & 64.0 \\
\hline Scheduled amortization ${ }^{5}$ & -8.3 & -7.5 & -7.5 & -14.5 & -32.4 & -33.9 & -46.5 & -52.8 & -57.0 \\
\hline SDR allocation & & & 100.7 & & & & & & \\
\hline Other capital ${ }^{6}$ & -58.0 & -102.0 & 24.6 & -18.0 & -13.6 & -10.0 & -19.6 & -9.1 & -9.1 \\
\hline Capital and financial account balance & 196.7 & 316.1 & 512.2 & 454.0 & 494.0 & 370.9 & 321.7 & 326.2 & 338.3 \\
\hline Errors and omissions & -5.4 & -21.3 & 8.2 & 0.0 & 0.0 & 0.0 & 0.0 & 0.0 & 0.0 \\
\hline Overall balance & 107.9 & 64.7 & 138.9 & 3.5 & -43.1 & 9.9 & 1.5 & 32.5 & 40.0 \\
\hline Financing & -107.9 & -64.7 & -138.9 & -3.5 & 43.1 & -9.9 & -1.5 & -32.4 & -40.0 \\
\hline Change in net foreign assets of NBR (increase -) & -107.9 & -64.7 & -138.9 & -3.5 & 43.1 & -9.9 & -1.5 & -32.4 & -40.0 \\
\hline Net credit from the Fund & 3.5 & 3.6 & 3.6 & -0.1 & -0.6 & -1.0 & -1.7 & -2.4 & -2.9 \\
\hline Disbursements/purchases & 3.5 & 3.6 & 3.6 & 0.0 & 0.0 & 0.0 & 0.0 & 0.0 & 0.0 \\
\hline Repayments/repurchases & 0.0 & 0.0 & 0.0 & -0.1 & -0.6 & -1.0 & -1.7 & -2.4 & -2.9 \\
\hline Change in other gross official reserves (increase -) & -112.7 & -44.1 & -138.8 & -9.0 & 43.7 & -8.9 & 0.3 & -30.0 & -37.1 \\
\hline Change in other foreign liabilities (increase +) & 1.4 & -24.2 & -3.7 & 5.6 & 0.0 & 0.0 & 0.0 & 0.0 & 0.0 \\
\hline Change in arrears (decrease -) & 0.0 & 0.0 & 0.0 & 0.0 & 0.0 & 0.0 & 0.0 & 0.0 & 0.0 \\
\hline Exceptional financing & 0.0 & 0.0 & 0.0 & 0.0 & 0.0 & 0.0 & 0.0 & 0.0 & 0.0 \\
\hline Financing need & 0.0 & 0.0 & 0.0 & 0.0 & 0.0 & 0.0 & 0.0 & 0.0 & 0.0 \\
\hline Identified financing & 0.0 & 0.0 & 0.0 & 0.0 & 0.0 & 0.0 & 0.0 & 0.0 & 0.0 \\
\hline Financing gap & 0.0 & 0.0 & 0.0 & 0.0 & 0.0 & 0.0 & 0.0 & 0.0 & 0.0 \\
\hline \multicolumn{10}{|l|}{ Memorandum items: } \\
\hline \multicolumn{10}{|l|}{ Current account deficit (percent of GDP) } \\
\hline Excluding official transfers & -11.9 & -14.4 & -17.3 & -19.3 & -18.6 & -14.8 & -12.9 & -11.6 & -10.6 \\
\hline Including official transfers & -2.2 & -4.9 & -7.3 & -7.9 & -8.8 & -5.4 & -4.4 & -3.7 & -3.5 \\
\hline Gross official reserves (including SDR allocation) & 552.4 & 596.4 & 735.3 & 744.3 & 700.6 & 709.4 & 709.2 & 739.2 & 776.3 \\
\hline Gross official reserves (months of imports of G\&NFS) & 4.7 & 4.8 & 5.1 & 4.8 & 4.7 & 4.7 & 4.4 & 4.3 & 4.2 \\
\hline Overall balance (percent of GDP) & 2.9 & 1.4 & 2.6 & 0.1 & -0.7 & 0.1 & 0.0 & 0.4 & 0.5 \\
\hline
\end{tabular}

Sources: Rwandan authorities; and IMF staff estimates and projections.

${ }^{1}$ Revision of methodology resulted in sharp increase of tourism revenues from 2008 , thus increasing export of services.

${ }^{2}$ Including interest due to the IMF.

${ }^{3}$ Current transfers include disbursed budgetary and HIPC grants and humanitarian and technical assistance.

${ }^{4}$ Includes project and budgetary loans.

${ }^{5}$ Excluding payments to the IMF.

${ }^{6}$ Other capital includes long-term private capital, commercial credit, change in the net foreign assets of commercial banks, and unrecorded imports. 
Table 6. Rwanda: Millennium Development Goals

\begin{tabular}{|c|c|c|c|c|c|}
\hline & 1990 & 1995 & 2000 & 2005 & 2008 \\
\hline \multicolumn{6}{|l|}{ Goal 1: Eradicate extreme poverty and hunger } \\
\hline Employment to population ratio, $15+$, total $(\%)$ & 87 & 87 & 84 & 80 & 80 \\
\hline Employment to population ratio, ages $15-24$, total (\%) & 79 & 80 & 73 & 66 & 64 \\
\hline GDP per person employed (constant 1990 PPP \$) & .. &.. &.. & .. & .. \\
\hline Income share held by lowest $20 \%$ & .. & .. & 5 & .. & .. \\
\hline Malnutrition prevalence, weight for age (\% of children under 5 ) & 24 & .. & 20 & 18 & .. \\
\hline Poverty gap at $\$ 1.25$ a day (PPP) (\%) & .. & .. & 38 & .. & .. \\
\hline Poverty headcount ratio at $\$ 1.25$ a day (PPP) (\% of population) & .. &.. & 77 & .. & .. \\
\hline Vulnerable employment, total (\% of total employment) &.. & 93 &.. & .. & .. \\
\hline \multicolumn{6}{|l|}{ Goal 2: Achieve universal primary education } \\
\hline Literacy rate, youth female ( $\%$ of females ages $15-24)$ & 75 &.. & 77 & .. & 77 \\
\hline Literacy rate, youth male ( $\%$ of males ages $15-24)$ & 75 & .. & 79 & .. & 77 \\
\hline Persistence to last grade of primary, total (\% of cohort) & .. & .. & 27 & 31 & .. \\
\hline Primary completion rate, total ( $\%$ of relevant age group) & 37 &.. & 22 & 40 & 54 \\
\hline Total enrollment, primary (\% net) & .. &.. & 76 & 84 & 96 \\
\hline \multicolumn{6}{|l|}{ Goal 3: Promote gender equality and empower women } \\
\hline Proportion of seats held by women in national parliaments (\%) & 17 & 17 & 17 & 49 & 56 \\
\hline Ratio of female to male primary enrollment (\%) & 92 & .. & 97 & 104 & 101 \\
\hline Ratio of female to male secondary enrollment (\%) & 74 & .. & 94 & 90 & 90 \\
\hline Ratio of female to male tertiary enrollment $(\%)$ & .. & .. & 48 & 62 & .. \\
\hline Share of women employed in the nonagricultural sector (\% of total nonagricultural employment) & .. & 32.4 & 33.0 & .. & .. \\
\hline \multicolumn{6}{|l|}{ Goal 4: Reduce child mortality } \\
\hline Immunization, measles ( $\%$ of children ages $12-23$ months) & 83 & 84 & 74 & 89 & 92 \\
\hline Mortality rate, infant (per 1,000 live births) & 106 & 132 & 112 & 85 & 72 \\
\hline Mortality rate, under-5 (per 1,000$)$ & 174 & 225 & 186 & 136 & 112 \\
\hline \multicolumn{6}{|l|}{ Goal 5: Improve maternal health } \\
\hline Adolescent fertility rate (births per 1,000 women ages $15-19$ ) & .. &.. & 50 & 40 & 36 \\
\hline Births attended by skilled health staff ( $\%$ of total) & 26 & .. & 31 & 39 & 52 \\
\hline Contraceptive prevalence (\% of women ages $15-49)$ & 21 & 14 & 13 & 17 & 36 \\
\hline Maternal mortality ratio (modeled estimate, per 100,000 live births) & .. &.. &.. & 1,300 & .. \\
\hline Pregnant women receiving prenatal care $(\%)$ & 94 & .. & 92 & 94 & 96 \\
\hline Unmet need for contraception ( $\%$ of married women ages $15-49$ ) & 39 &.. & 36 & 38 & .. \\
\hline \multicolumn{6}{|l|}{ Goal 6: Combat HIVIAIDS, malaria, and other diseases } \\
\hline Children with fever receiving antimalarial drugs ( $\%$ of children under age 5 with fever) & .. &.. & 13 & 12 & .. \\
\hline Condom use, population ages $15-24$, female (\% of females ages $15-24)$ & .. & .. & 3 & 5 & .. \\
\hline Condom use, population ages $15-24$, male (\% of males ages $15-24)$ & .. & .. & 24 & 19 & .. \\
\hline Incidence of tuberculosis (per 100,000 people) & 167 & 241 & 348 & 418 & 387 \\
\hline Prevalence of HIV, female (\% ages $15-24)$ &.. & .. &.. & 1.4 & 1.4 \\
\hline Prevalence of HIV, male (\% ages $15-24)$ & .. & .. &.. & 0.5 & 0.5 \\
\hline Prevalence of HIV, total (\% of population ages $15-49$ ) & 9.2 & 7.0 & 4.7 & 3.1 & 2.8 \\
\hline Tuberculosis case detection rate (all forms) &.. & 33 & 31 & 26 & 26 \\
\hline \multicolumn{6}{|l|}{ Goal 7: Ensure environmental sustainability } \\
\hline $\mathrm{CO} 2$ emissions (kg per PPP \$ of GDP) & 0 & 0 & 0 & 0 & 0 \\
\hline $\mathrm{CO} 2$ emissions (metric tons per capita) & 0 & 0 & 0 & 0 & 0 \\
\hline Forest area ( $\%$ of land area) & 12.9 & 13.4 & 13.9 & 19.5 & 21.7 \\
\hline Improved sanitation facilities (\% of population with access) & 29 & 26 & 25 & 23 & 23 \\
\hline Improved water source (\% of population with access) & 65 & 64 & 65 & 65 & 65 \\
\hline Marine protected areas (\% of total surface area) &.. &.. &.. & .. & 0 \\
\hline Terrestrial protected areas ( $\%$ of total surface area) &.. &.. &.. & .. & 8 \\
\hline \multicolumn{6}{|l|}{ Goal 8: Develop a global partnership for development } \\
\hline Net ODA received per capita (current US\$) & 40 & 128 & 40 & 64 & 96 \\
\hline Debt service (PPG and IMF only, \% of exports, excluding workers' remittances) & 10 & 20 & 25 & 4 & 1 \\
\hline Internet users (per 100 people) & 0.0 & 0.0 & 0.1 & 0.6 & 3.1 \\
\hline Mobile cellular subscriptions (per 100 people) & 0 & 0 & 0 & 2 & 14 \\
\hline Telephone lines (per 100 people) & 0 & 0 & 0 & 0 & 0 \\
\hline \multicolumn{6}{|l|}{ Other } \\
\hline Fertility rate, total (births per woman) & 7 & 6 & 6 & 6 & 5 \\
\hline GNI per capita, Atlas method (current US\$) & 340 & 230 & 250 & 250 & 440 \\
\hline GNI, Atlas method (current US\$) (billions) & 2.4 & 1.3 & 2.0 & 2.3 & 4.3 \\
\hline Gross capital formation (\% of GDP) & 14.6 & 13.4 & 18.3 & 21.6 & 24.1 \\
\hline Life expectancy at birth, total (years) & 33 & 29 & 43 & 48 & 50 \\
\hline Literacy rate, adult total ( $\%$ of people ages 15 and above) & 58 & .. & 65 & .. & 70 \\
\hline Population, total (billions) & 0.0 & 0.0 & 0.0 & 0.0 & 0.0 \\
\hline Trade ( $\%$ of GDP) & 19.7 & 31.0 & 34.4 & 36.9 & 46.1 \\
\hline
\end{tabular}

Source: World Development Indicators

Figures in italics refer to periods other than those specified. 


\section{APPENDIX I. LETTER OF INTENT}

Kigali, Rwanda

May 25, 2010

Mr. Dominique Strauss-Kahn

Managing Director

International Monetary Fund

$70019^{\text {th }}$ Street, N.W.

Washington, D.C., 20431

Dear Mr. Strauss-Kahn,

1. The attached Memorandum summarizes the economic and financial policies of the government of Rwanda for the period June 30, 2010-June 30, 2013. The government requests that these policies, which aim at macroeconomic stability, laying the foundation for sustainable growth, reducing poverty, and deepening fiscal and financial sector reforms, be supported by the International Monetary Fund (IMF) under a three-year Policy Support Instrument (PSI).

2. A PSI is, in the government's view, the appropriate vehicle at the present time to maintain a close policy dialogue with the IMF and signal commitment to sound policies to the international community. In the government's view, support under a PRGF ${ }^{1}$ would not be appropriate as Rwanda currently has no IMF financing needs. Rwanda also has a long record of macroeconomic stability that the government aims to safeguard through the implementation of the policies set out in the attached Memorandum. The government is determined to adhere to the timelines for implementation of the program, as laid out in the Memorandum, and comply with the semi-annual review schedule under the PSI.

3. The government believes that the policies and measures set forth in the attached Memorandum are adequate to achieve the objectives of a PSI program. Given its commitment to these objectives, it will promptly take any additional measures necessary for their achievement. The government will consult with the IMF - at its own initiative or whenever the Managing Director of the IMF requests such a consultation-before the adoption of any such measures or changes to the policies described in the attached Memorandum.

4. The government will provide the Fund with such information as the Fund may request in connection with the progress made in implementing the economic and financial policies and achieving the objectives of the program.

\footnotetext{
${ }^{1}$ Now known as Extended Credit Facility (ECF).
} 
5. The Government of Rwanda authorizes the publication and distribution of this letter, its attachments, and all reports prepared by Fund staff regarding this request for PSI.

Sincerely yours,

$/ \mathrm{s} /$

John Rwangombwa

Minister of Finance and Economic Planning
François Kanimba

Governor, National Bank of Rwanda 


\section{Attachment I. \\ Memorandum of Economic and Financial Policies \\ of the Government of Rwanda \\ May 25, 2010}

\section{INTRODUCTION}

1. The Government of Rwanda remains committed to achieving sustained economic growth and poverty reduction and consolidating macroeconomic stability. The strategies to achieve these goals are set out in the Economic Development and Poverty Reduction Strategy (EDPRS) for 2008-12 $2^{2}$ as well as in the Vision 2020 development plan. The Government and the International Monetary Fund (IMF) are cooperating on a three-year Policy Support Instrument (PSI) to support prudent macroeconomic policies and structural reforms that underlie strategies in the EDPRS and Vision 2020.

2. This memorandum of economic and financial policies (MEFP) reviews performance during 2009 and the first quarter of 2010 and describes policies and targets through end-June 2011 and the medium term.

\section{ECONOMIC DEVELOPMENTS AND REFORMS IN 2009-10}

\section{Performance against targets agreed with Fund staff has been broadly} satisfactory. $^{3}$ At end-2009, all but two assessment criteria were met (Table 1). Net credit to the government was missed due to delays in disbursement of budgetary grants (World Bank and Education Fast Track Initiative), a shortfall of US\$78 million which has since been disbursed. The zero ceiling on nonconcessional external borrowing was also missed due to US\$13.1 million borrowing for the government-owned airline, Rwandair.

\section{Growth}

4. After a strong growth in 2008, economic activity slowed in 2009. The provisional estimates of the National Institute of Statistics of Rwanda (NISR) indicate that real GDP in 2009 grew at 6 percent compared to 11.6 percent in 2008. Growth was strong in the early part of the year, thanks to a strong agricultural harvest including from ongoing investments in the sector. However, the impact of the global financial crisis and tight liquidity conditions was felt in Rwanda especially in the second half of 2009, as contributions from the industrial

\footnotetext{
${ }^{2}$ Rwanda's latest EDPRS (IMF Country Report No. 08/90) and Joint Staff Advisory Note (IMF Country Report No. 08/91) were issued to the Executive Board on February 14, 2008. An Annual Progress Report is under preparation and is expected to be made available to the IMF and other partners before the second review of the PSI.

${ }^{3}$ Before the expiration of the PRGF arrangement in August 2009, the government and IMF staff agreed on a macroeconomic framework for 2009/10, with indicative targets for monitoring purposes.
} 
and service sectors - worst hit by the crisis - declined significantly. CPI inflation (end of period) stood at 5.7 percent in 2009 compared to 22.3 percent in the previous year partly reflecting lower import prices.

\section{Fiscal policy}

\section{During the first half of the $2009 / 10$ fiscal year, ${ }^{4}$ the overall fiscal deficit} (including grants) was some 0.5 percent of GDP lower than budgeted mainly on account of lower spending. Total expenditure and net lending was 0.4 percent of GDP below the budget, with savings in both current and capital spending. Spending on domesticallyfinanced capital projects registered the largest shortfall ( 0.7 percent of GDP) mainly as a result of delays in completing tender documents, although there has been a recovery during the first quarter of 2010. On the other hand, net lending was higher-than-budgeted from outlays to Rwandair and the Kigali International Convention Complex-projects that are among the government's strategic investment projects to boost economic growth (see 923 ). Domestic revenue collection was slightly higher-than-expected, by 0.1 percent of GDP. Higher collection of direct and indirect taxes offset shortfalls in international trade taxes and non-tax revenues (due mainly to lower recovery of fertilizer receipts and ID cards).

\section{In March 2010 the parliament approved a revised budget for FY2009/10 that} reflects a higher resource envelope matched by higher spending, leaving the overall fiscal balance unchanged. Total resources (revenues and grants) are now budgeted to be higher by Rwf 36 billion (1.1 percent of GDP). Total grants are higher by Rwf 52 billion (1.7 percent of GDP), mainly budget support grants in response to the adverse effects of the global economic downturn on Rwanda. On the other hand, domestic revenues are lower than the original budget, mainly non-tax revenues from continued expected losses in recovery of fertilizer receipts and driving license fees. The higher spending reflects the higher net lending already committed, as well as higher current spending to fund one laptop per child and nine-year basic education policies.

\section{In the area of tax administration, the Rwanda Revenue Authority (RRA)} continued its efforts to decentralize its services expand the tax base and increase compliance. In 2009, RRA established three district branches in Kigali City (block management system) which provide headquarter services (tax payer education, tax payer registration, audit and enforcement) to taxpayers. In addition, taxes can now be paid at some designated commercial banks. In 2009, about 1,257 new taxpayers were registered between September and December 2009, bringing the total number of tax payers to 34,193 at end-2009.

\footnotetext{
${ }^{4}$ Rwanda's fiscal year runs from July to June.
} 
8. The RRA has begun to address the collection of outstanding tax arrears. Our existing enforcement efforts enabled us to recover some RWF 10 billion in arrears in 2009 ( 0.3 percent of GDP). To further improve collection of tax arrears in a sustainable manner, RRA developed its Debt Management Strategy (RRADMS) in November 2009, an automated management module for tax arrears. At end-2009 total outstanding arrears stood at Rwf 39 billion (1.3 percent of GDP), of which Rwf 27 billion in old and difficult arrears will be written off.

\section{With regard to the current PFM reform strategy, a number of key milestones} outlined below have either been completed or are on track to be completed by end-June 2010:

- Budget calendar aligned with practices in the EAC (July-June fiscal year) (completed July 2009);

- Established Public Investment Program (PIP) and Public Private Partnership (PPP) units within MINECOFIN. Staffing of the units is expected to be completed by end-June 2010;

- Finalize fiscal decentralization strategy by May 2010;

- Strengthening internal controls for financial management and reporting in Ministries, Departments and Agencies and Sub-National governments is ongoing via on-the-job training and sponsorship of professional courses such as the Association of Chartered Certified Accountants (ACCA- 90 staff currently enrolled) and Certified Financial Analyst (CFA- 1 staff currently enrolled);

- MTEF (Medium-Term Expenditure Framework) was successfully integrated into the budget process for the 2009/2010 fiscal year. Capacity building in implementing MTEF, budget preparation, and sector costing was carried out for the staffs in National Budget and Development Planning and Research Units and later extended to all budget officers. Additionally, all externally financed projects and programs managed by the Government are fully reflected in the budget;

- Public Expenditure Reviews (PERs) are being carried out in the Water \& Sanitation, Agriculture, Education and Social Protection sectors and will be completed by June 2010;

- A fully fledged Government Portfolio Unit was set up during 2009/2010 and on-the-job training to impart financial analysis skills to the staff is ongoing with an aim of improving performance monitoring of the Government Business Entities (GBEs) and its financial management and reporting, based on government financial systems and regulations and relevant international best practices. Additionally, plans are underway to provide training to Director Generals and Board members of GBEs in financial management, reporting, risk management and corporate governance by end-June 2010; 
- On-the- job training for internal audit staff commenced in March 2010. This is aimed at building the capacity of internal auditors to conduct payroll, financial, value-for-money (VFM), and systems and risk based audits;

- Restructuring of the Rwanda Public Procurement Authority (RPPA) was approved by cabinet in November 2009 and is in progress. The restructuring was necessitated by the need to enable RPPA to exit from high value procurement operations and contract approval to pure regulatory and capacity building functions in line with international best practice;

- Greater efforts have been made towards increasing public access to fiscal data, with the Ministry of Finance website (http://www.minecofin.gov.rw) being utilized to disseminate information (e.g. budget guidelines are posted throughout the budgeting cycle, budget speeches, guidelines to the budget, Government economic reports, and budget execution data are posted on an annual basis).

- Progress has been made on the establishment of the PFM Reform Secretariat, although delays have been experienced. The PFM Reform Coordinator was recruited in November 2009 and the procurement specialist was recruited in January 2010, and the recruitment of an M\&E Specialist, Procurement Assistant and Accountant was completed in April 2010. The efficiency of the Secretariat is expected to greatly improve now that all the key personnel are in place.

\section{Monetary and exchange rate policies}

10. Monetary policy in $\mathbf{2 0 0 9}$ addressed the persistence of high inflation from $\mathbf{2 0 0 8}$ as well as the tight liquidity conditions. The global food and fuel crises in 2008 posed a number of challenges to monetary policy implementation in Rwanda, as these shocks became embedded in underlying inflation and inflation expectations, with inflation rising to 22.3 percent at end-2008. This episode was followed by a domestic liquidity crisis that started in late 2008 and continued into the first half of 2009, following a significant withdrawal of bank deposits by two large institutional depositors. The resulting maturity mismatch on banks' balance sheets contributed to a sharp decline in growth of commercial banks' credit to the private sector from 73 percent in 2008 to 5.7 percent in $2009 .{ }^{5}$ During the period, the NBR conducted a prudent monetary policy, controlling reserve money, in order to avoid exacerbating the exogenous price shocks with excessive money supply.

\section{The NBR and the government responded by putting in place a combination of} measures to improve liquidity and stimulate private sector credit, including: reducing the reserve requirements from 8 percent to 5 percent in February 2009; suspending roll-over

\footnotetext{
${ }^{5}$ Credit to the private sector in 2009 includes credit from Credit and Saving Scheme - Zigama (CSS - Zigama), a newly licensed bank. CSS, a large savings and credit cooperative, was given a license to operate as a bank in 2010. However, in line with standard procedures, NBR has included CSS in the monetary survey for 2009 , for benchmarking purposes. Excluding CSS, growth in credit to the private sector was minus 1.7 percent in 2009.
} 
of Treasury Bills that matured during the year; making available to commercial banks new liquidity facilities by the NBR and the Government; reduction of the Key Repo Rate (NBR's policy rate) from 9 percent to 7.5 percent in December 2009, followed by a further decline to 7 percent in March 2010.

12. The monetary policy measures helped to improve the liquidity conditions. The sharp reduction in deposits following the liquidity tightening pushed commercial banks to increase deposit rates significantly, restoring positive real interest rates so as to attract deposits. As a result, the average commercial bank deposit rate rose from 5.97 percent at the beginning of 2008 to 8.54 percent by the end of 2009 . These, together with the measures implemented by the NBR and the Government, began to yield results at the end of 2009 with early signs of a turnaround in liquidity conditions as broad money picked up in the second half of 2009. Also credit to the private sector started to recover towards the end of the year. Inflation at the same time fell into single digits (5.7 percent at the end of 2009) and 2.5 percent at end-February 2010, reflecting the fall in commodity prices and slowing down of economic activity.

13. Improvement has been made in the area of liquidity forecasting notably through the coordination of fiscal and monetary policies. In this regard, Treasury Management Committee (TMC) meetings have been regularly held to discuss the impact of government operations on the banking liquidity situation and coordination of fiscal and monetary policies.

14. The real effective exchange rate continued to depreciate in 2009. Movements in the real effective exchange rate (a depreciation of 8.3 percent in 2009) has been driven mainly by movements in inflation differentials as the nominal exchange rate has remained virtually unchanged in the period, with an annual effective depreciation of 2.8 percent in 2009.

\section{With a view to encourage the development of the interbank market, NBR} decided to reform its intervention mechanism in the foreign exchange market in such a way to avoid unnecessary competition with interbank transactions. Since September 2009, NBR has intervened to sell foreign exchange on demand to banks at the NBR selling rate (average reference rate) ${ }^{6}$ and based on pre-set criteria ${ }^{7}$. As a further improvement, since

\footnotetext{
${ }^{6}$ From January 16th, 2009 to January 11th, 2010, ARR was calculated as the ten days weighted moving average rate of customer deals transacted by both banks and NBR, with buying and selling rates set at $0.6 \%$ margin on either side of the ARR. With effect from January 12th, 2010, the NBR's average reference rate has changed to 5 days moving average.

${ }^{7}$ The criteria for NBR intervention are: (i) the foreign exchange net position in the banking system is at least 2 percent; (ii) at least half of the banks are in negative position; and (iii) at least two of the banks had expressed the need for NBR intervention.
} 
March 24, 2010, NBR foreign exchange sales are based on interbank rates and not the NBR selling rate. The timing and rate of NBR foreign exchange sales are announced at the close of business the day before each operation. The new policy which was communicated to banks on March 23, 2010, and aims at stimulating further the already growing foreign currency interbank market and introducing more flexibility in the foreign exchange rate policy.

\section{Financial sector policies}

16. Significant progress has been made in $\mathbf{2 0 0 9}$ in deepening the financial sector. The number of people using formal financial services rose to 2 million in 2009, from 1.4 million in 2007 and 1.8 million in 2008. Also during 2009, the NBR licensed one new commercial bank, 10 new microfinance institutions, and 22 new commercial bank branches, bringing the total number of bank branches to 105 country-wide.

17. To further deepen the domestic financial market, the NBR entered into a SWAP agreement with the International Finance Corporation (IFC). The agreement signed on November 8, 2009, will enable the IFC to provide local currency loans to the private sector. Through this SWAP agreement, IFC will make available foreign exchange resources (up to US\$50 million) to NBR at a pre-determined exchange rate and the NBR will make the equivalent local currency available to the IFC for onward disbursement to the private sector at a variable interest rate using local banks. The foreign currency will be recorded at the NBR only as the loans to the private sector are finalized. The foreign currency for this and any subsequent foreign currency swap agreements will be escrowed at the NBR against repayment of the loans. This SWAP arrangement is expected to augment credit delivery to sectors of the economy that currently lack long-term local currency financing such as infrastructure, SMEs, and Manufacturing.

18. However, 2009 was a challenging year for commercial banks. The liquidity crisis coupled with deterioration in asset quality affected banks' profitability significantly although banks remained well capitalized. In 2009, banks wrote off some RWF 27.3 billion in non-performing loans, up from RWF 26.9 billion in 2008. Non-performing loans increased from 12.6 percent of gross loans in December 2008 to 13.1 percent in December 2009. Return on equity decreased from 18.5 percent to 5.3 percent and return on assets decreased from 2.4 percent to 0.7 percent during the same period. The consolidated capital adequacy ratio for commercial banks increased from 15.9 percent in December 2008 to 19.7 percent in December 2009.

\section{The NBR strengthened its risk-based prudential supervision policies to} safeguard the soundness of the financial system. However, capacity constraints at the NBR, including high turnover of skilled and experienced bank examiners impacted on the scope of banking supervision. In 2009, only four onsite inspections were conducted (out of 10 planned), of which one was undertaken with the assistance of a senior inspector from the Central Bank of Kenya. However, during the same period, the frequency and efficiency of 
off-site surveillance was maintained, including regular meetings with the managements of all financial institutions.

20. The NBR also adopted a number of regulatory and institutional reforms to further strengthen the supervision of microfinance (MFIs) and non-bank financial institutions (NBFI's). A new insurance law was passed in March 2009 to better monitor the insurance sector and facilitate the development of the domestic capital markets. Regarding the MFIs, the NBR created a new department tasked with supervising MFIs within the Financial Stability Directorate. The regulatory guidelines for MFIs were published in the Official Gazette in July 2009 and the implementation of this legal and regulatory framework has already started. Also in the area of microfinance, the Government adopted the "UMURENGE SACCO" Program in March 2009 with the objective of establishing at least one Savings and Credit Cooperatives (SACCO) per UMURENGE (the smallest administrative unit).

\section{External sector}

21. The external current account deficit widened from $\mathbf{5}$ percent of GDP in 2008 to 7 percent in 2009. Lower exports and higher imports have contributed to a larger deterioration in the trade deficit in 2009. Exports have been lower than expected (mainly minerals) reflecting the continued lagged effects of weak global demand, but more importantly imports are significantly higher than expected. Higher than programmed foreign direct investment (FDI), higher private remittances and foreign aid and reduced import barriers within the East African Community (EAC) and COMESA (Common Market for Eastern and Southern Africa) contributed to an increase in imports, especially for consumer goods and capital goods. Higher than expected current transfers and capital inflows contributed to an overall balance of payments surplus for 2009 and a consequent increase in gross international reserves and improvement in import coverage to 6.2 months.

\section{OBJECTIVES AND ECONOMIC POLICIES FOR THE THREE-YEAR PSI PERIOD}

\section{Our overriding economic objective is to sustain real GDP growth rates of}

6-7 percent a year. Continued macroeconomic stability is a necessary condition for meeting this challenge. The government's economic policies during the PSI period aim to consolidate macroeconomic stability by:

- establishing a sustainable fiscal position, with strong revenue efforts needed to reduce Rwanda's aid dependency;

- strengthening monetary and exchange rate policies to ensure low and stable inflation; and;

- Pursuing structural reforms that deepen the financial sector, diversify the export base, and improve the business environment. 


\section{Achieving the growth objectives also requires a scaling up of infrastructure}

investment. The government's strategic investment plan aims to alleviate critical infrastructure constraints to increasing exports of goods and services and improve competitiveness and growth, consistent with the objectives of the government's Economic Development and Poverty Reduction Strategy (EDPRS). The investments are outlined below:

- Broaden energy access to increase household grid connections from 6 percent at end-2008 to 16 percent by 2013, and to reach all public health centers and administrative offices, and connect half of all schools, consistent with the EDPRS objectives.

- Develop high-capacity fiber optic line to provide broadband wireless technology for up to 100,000 users by mid-2010, and connect Rwanda to undersea fiber-optic cables by 2013. This will reduce broadband purchase costs by some 90 percent compared to using satellite technology.

- Build and rehabilitate an international railway connection, together with the governments of Burundi and Tanzania. The initiative has the potential to substantially reduce transportation costs.

- Build a new airport at Bugesera that conforms to strict international standards and has the capacity to turn Rwanda into a Central African hub and gateway.

- Expand Rwandair (the national air carrier) as a complement to the airport project, in order to create links for high-value exports and regional and international air travel. It is expected that Rwandair will be a viable business by end-2014, and be ready for divestment and privatization. The feasibility study indicates a rate of return of 11 percent by 2014.

- Build a convention center together with a five-star hotel, in order to tap into the lucrative convention and business tourism markets. Construction started in 2009 and the center is expected to be open for business by end-2011. The feasibility study indicates a rate of return of about 13 percent from 2012.

\section{The PSI supports poverty reduction and growth objectives outlined in our} EDPRS (2008-12). The key economic policies of the PSI - to consolidate macroeconomic stability and scale up investments - are in line with the strategic pillars of the EDPRS and are designed to ensure pro-poor growth-for-jobs-and-exports, which are critical for poverty reduction.

\section{The external current account deficit (including grants) is expected to worsen} slightly to 7.9 percent in $\mathbf{2 0 1 0}$, from the increased imports of capital goods associated with the large planned investment projects, before declining over the medium term. Exports, both traditional and nontraditional, are also expected to improve gradually in response to recovery in global demand and increased domestic productivity. Foreign direct investment and other capital inflows are also expected to recover in response to the improved investment climate in order to provide some of the required funds for imports. Official 
assistance is projected to decline slightly over the medium term, as the country reduces its dependence on donor aid. Some draw down of international reserves will be necessary, but gross reserves should remain at comfortable levels (not less than 4 months of imports and services) during the three-year PSI period.

26. Fiscal policy will balance the competing objectives of meeting our growth and poverty reduction objectives as envisaged in the EDPRS whilst preserving mediumterm fiscal and debt sustainability. The overall fiscal deficit (incl. grants) will decline from 1.1 percent of GDP in FY2009/10 to 0.6 percent of GDP in FY2012/13, reflecting domestic revenue mobilization, as well as lower spending. Increases in domestic revenues of 2 percent of GDP over the PSI period are front loaded, underpinned by revenue administration measures. Our fiscal strategy also envisages prioritization of expenditures to accommodate some additional spending associated with some of the six government strategic investment projects (see para 23). Grant finance is projected to remain high over the period though a gradual reduction is envisaged. Further reforms in revenue administration and PFM will underpin the medium-term fiscal performance.

27. The National Bank of Rwanda (NBR) is committed to making monetary and exchange rate policies more proactive to respond to changing fundamentals in order to maintain low and stable inflation. The NBR will aim for 7 percent inflation (12 month rate) at end 2010 and 5 percent over the medium term, consistent with a gradual recovery in domestic, regional and world economies, as well as current expectation of oil and food price developments.

28. Our structural reform agenda intends to support a growing private sector and reduce vulnerabilities arising from our narrow export base. We will continue to improve the business environment - building on our achievement in 2009 as the global leader in reducing the cost of doing business, according to the World Bank's worldwide ranking of business regulations - promulgate laws to strengthen the legal and regulatory framework towards the development of the domestic capital market, and deepen the financial sector. We will complement these efforts with our export strategy that intends to reduce our dependence on coffee, tea and minerals as main source of exports.

\section{Growth}

\section{THE POLICY AGENDA FOR 2010/11}

29. Under the program, Real GDP growth of 5.4 percent is projected for 2010, with a further pick up to 5.9 percent in 2011, though we expect to achieve higher average growth rates of about 7 percent over the period. Growth in 2010 will be led by a recovery in services, the sector hardest hit by the global downturn, as well as the agricultural sector which will continue to benefit from increased investments in fertilizers, improved seeds and extension services. Already, the season A harvest for early 2010 is showing successes from this strategy. Agriculture is expected to grow by about 6 percent in 2010 and 2011, close to 
the past five- year average. The expected recovery in the global economy and pickup in commodity prices would also increase aggregate demand for Rwanda's exports, thus further sustaining growth.

\section{Fiscal policy}

30. The FY20010/11 budget envisages overall fiscal deficit (incl. grants) to widen by 2.5 percent of GDP to about 3.6 percent of GDP, despite unchanged expenditures and higher domestic revenue mobilization. The higher deficit reflects a decline in grants from the unusually high level in 2009/10. The higher overall deficit will be financed mainly by an increase in domestic borrowing (2.3 percent of GDP). Although overall spending remains somewhat elevated (rising slightly to 27 percent of GDP), composition of spending is tilted toward investment spending. Re-current spending declines by 0.3 percent of GDP while domestically-financed capital spending rises by 1.5 percent of GDP, to 6.3 percent of GDP in FY2010/11, reflecting the government effort to devote more resources to strategic investment projects. The government is committed to keep priority expenditures, consistent with the EDPRS, at 13 percent of GDP. 
Priority Expenditure as percent of FY GDP

\begin{tabular}{lrrr}
\hline & $2008 / 09$ & $2009 / 10$ & $2010 / 11$ \\
\hline Employee Cost & 1.9 & 1.8 & 1.7 \\
Goods and Services & 2.0 & 2.0 & 2.0 \\
Transfers and Subsidies & 4.0 & 4.5 & 4.5 \\
Exceptional Expenditures & 0.5 & 0.2 & 0.2 \\
Net Lending & 0.1 & 0.1 & 0.1 \\
Domestic Capital Expenditure & 4.5 & 4.5 & 4.5 \\
\hline Total & & & $\mathbf{1 3 . 0}$ \\
\hline Memo: & & & \\
$\quad$ Nominal GDP (FY basis) & 2,765 & 3,149 & 3,539
\end{tabular}

\section{Domestic revenues will rise by 1.4 percent of GDP in FY2010/11, underpinned}

by further tax administration measures. The revenue targets are ambitious but the government is confident that they can be achieved. The underlying tax administration measures include:

- Submit to Cabinet for approval of draft legislation to transfer collection and audit functions of social security contributions (Pension and RAMA ${ }^{8}$ ) to RRA is planned for end-December 2010 (structural benchmark). This measure will reduce the burden incurred by taxpayers in meeting payroll obligations and broaden the tax base by bringing into the tax net potential taxpayers who make their social security contributions but do not pay other taxes and vice versa.

- Fully automate the services of the RRA District offices in Kigali City by March 2011 and roll-out to provinces. This will further improve RRA services to the taxpaying community, improve tax education, and widen the tax base.

- Automate the enforcement module in the Standard Integrated Government Tax Administration System (SIGTAS) by December 2010. This will help the tax administration improve enforcement efforts to recover outstanding arrears and stop

\footnotetext{
${ }^{8}$ La Rwandaise d'Assurance Maladie (RAMA).
} 
them from escalating. Old and irrecoverable arrears will be written off in accordance with the law.

- Improve risk-based assessment of ASYCUDA++ by introducing electronic submission of supporting customs documents by end-June 2010 (structural benchmark). This will help improve the speed and accuracy of customs verification of imported goods, improving the detection, prevention and monitoring of high risk transactions that cause revenue losses and security threat to citizens. Full automation will be completed over the medium term.

- Building on the success of the automated management module for tax arrears, RRA will collect an additional Rwf 7.6 billion ( 0.2 percent of GDP) in tax arrears by end-2011.

\section{Public Financial Management}

32. Implementation of Smartgov (a component of IFMIS) ${ }^{9}$ will be piloted in ministries, departments and agencies and local districts ${ }^{10}$ starting in July 2010 (structural benchmark). The pilot of the Smartgov software will begin with core modules: budgeting, payables, receivables, and general ledger. The pilot program has experienced delays as a result of deficiencies in the project management arrangements, system design, and functionality as highlighted during a Quality Assurance review conducted in April 2009. This pilot will determine if the software can be rolled out to all agencies.

33. Public Expenditure Reviews (PER) will be conducted for two more sectors (ICT and Transport) by end-March 2011 (structural benchmark). This will further improve expenditure allocation and efficiency, as well as medium-term expenditure budgeting and fiscal planning.

34. Reforms aimed at building capacity and enhancing the professionalism of staff at the Rwanda Public Procurement Authority (RPPA) is ongoing. To this effect, the Procurement User Guides and Code of Ethics in Public Procurement are being developed. Also a consultancy to build capacity among the members of the Independent Procurement Review Panel is planned to be undertaken in May 2010 and concluded in August 2010. In order to ensure sustainable capacity building efforts, arrangements are being made to establish a Procurement Training Centre at the School of Finance and Banking.

\footnotetext{
${ }^{9}$ Integrated Financial Management Information System (IFMIS).

10 Treasury Department, Ministry of Finance and Economic Planning, District of Nyarugenge, Kigali Institute of Education, and RSSP (Rural Sector Support Project).
} 
35. Plans are underway to introduce modern audit software in the offices of the Auditor General and Government Chief Internal Auditor and to oversee the establishment of audit committees in key budget agencies (using a piloting approach) by end-June 2011.

36. The next PEFA assessment is scheduled to take place between July 2010 and September 2010, and the results will inform the PFM agenda for the medium-term.

37. The fiscal decentralization strategy is scheduled to commence during the 2010/2011 fiscal year, paving the way for the devolution of financial resources and decisionmaking powers to sub-national governments. The district tax management software is currently being piloted in Kigali district sectors and will be rolled out once the pilot phase is successful.

38. The government is considering a Public Private Partnership (PPP) model for some of its strategic investments to ensure efficient management, crowd in the private sector and manage investment costs and risks. To this end, the Government has developed draft PPP guidelines that set the institutional framework and procedures for PPPs. The government will also establish, by September 2010, a Public Investment Committee whose role will be, among others, to ensure that the fore-mentioned guidelines and procedures are respected. Furthermore, a legal and regulatory framework for PPP is being developed and due to be submitted to Cabinet by end-2010. The government will consult with the Fund before implementing a project under a PPP, in particular if it implies any new contingent liabilities for the government.

\section{Debt Management}

39. A revised public debt policy and medium-term debt strategy (MTDS) will be submitted to cabinet for approval by end-December 2010 (structural benchmark). The debt management strategy will develop a clear framework for borrowing, establishing the principles that should guide decisions regarding the portfolio mix of debt, currency composition, maturity, interest rates and other risks of debt portfolio. During the PSI period, all avenues for concessional financing will be explored, but some nonconcessional borrowing may be needed to support the implementation of the government's six strategic investment projects. The government will limit the contracting or guaranteeing of new nonconcessional debt to possible debt guarantees for the Kigali Convention Center (KCC) and Rwandair, two of the six strategic investments. The amount of nonconcessional borrowing for this purpose should not exceed US\$240 million during the period June 30, 2010-June 30, 2013. 


\section{Monetary and exchange rate policies}

40. Reserve money will continue to remain the anchor of the monetary program. Reserve money and broad money are projected to grow in line with nominal GDP growth. Growth in deposits in response to the net liquidity impact of the budget spending and higher commercial bank deposit rates will allow substantial increases in private sector credit. Private sector credit is projected to grow annually at least in line with nominal GDP growth.

41. NBR will target the average reserve money rather than the end-period reserve money as a way to smooth the path of liquidity. This will be done through an appropriate mix of monetary policy instruments, including foreign exchange sales (subject to the conditions of the foreign exchange market), and daily repos and reverse repos. To ensure that NBR does not jeopardize its profit and loss accounts, the budget will assume the sterilization costs.

42. The NBR will make greater use of monetary policy instruments - exchange rate flexibility, sterilization and interests rate - to keep inflation stable and in low single digits. To this end, the Monetary Policy Committee (MPC) of the NBR will meet quarterly to assess the state of the economy and sources of inflationary pressure, using the newly developed high frequency data on indicators of economic activity and business and consumer confidence surveys compiled by the NBR. Decisions on the policy rate (the key repo rate) would be made based on developments in the economic fundamentals. These decisionsaimed at making monetary policy more responsive to changes in fundamentals - enhance the effectiveness of monetary policy to achieve the overall objective of stable and low inflation.

43. We will improve our communication strategy in order to be effective at shaping the expectations of the market and the general public, and provide a forum for greater accountability of the NBR. Starting in June 2010, we will publish a report and put on the NBR website the underlying economic assessment supporting MPC decisions within a month following each quarterly MPC meeting (structural benchmark). The report would provide an assessment of economic fundamentals, communicate monetary policy decisions and explain their rationale.

44. To allow for greater exchange rate flexibility, the NBR is committed to putting in place an interbank exchange rate corridor and announce its introduction to all foreign exchange market participants, by end-June 2010, in line with planned MCM TA (structural benchmark). The corridor is a transition measure towards greater exchange rate flexibility and is aimed at encouraging interbank market development; and the NBR will only intervene at the extremes of the corridor.

45. The NBR intends to request a safeguard assessment to update the 2007 assessment and help in strengthening the Bank's safeguards and governance. Given the extensive changes in Rwanda's financial sector since the last bank-Fund Financial Sector 
Assessment (FSAP) in 2005, the government would also welcome a FSAP update at the earliest possible opportunity.

\section{Financial sector policies}

46. The NBR will continue to promulgate laws to strengthen the regulation of the financial sector. By end 2010, depending on the availability of technical assistance and the adoption of the pension law, the non-bank supervision department will embark on an onsite inspection of Caisse Sociale du Rwanda (CSR), the largest social security fund and pension provider in Rwanda. The NBR expects to finalize the remaining insurance regulations to implement the new insurance law before the end of 2011. Additionally, new insurance contract law and mandatory insurance law are in the drafting process and will be enacted in 2010 to 2012.

\section{The Financial Sector Development Plan (FSDP) is expected to be fully} implemented by June 2011. The multifaceted FSDP has the objective of strengthening and deepening the financial sector in Rwanda along the spectrum of microfinance to long term capital markets.

\section{The NBR is in the process of promulgating laws to facilitate financial} deepening, including a credit reference bureau. A law governing the organization of collective investment schemes (CIS) was drafted and handed over in June 2009 to the Capital Market Authority (CMA), the supervisor of the CIS. This draft law, together with two other CMA draft laws, is expected to be passed by the Parliament in May 2010. The legal framework to operate a private credit reference bureau has also been developed. The draft law is in Parliament and a new private credit bureau, CRB Africa, is going through the licensing process and will be operational before end May 2010. At the same time the Central Bank is improving the existing Public Credit Registry which will be used for supervisory purposes.

49. The NBR is committed to hiring and training new staff to develop capacity for the Banking Supervision Department. The objective over the PSI period is to be able to conduct on-site inspections for each bank at least once in a 24 month cycle (a total of eight commercial banks and 4 specialized banks in 2010). Given the time needed to fully train seasoned bank examiners, NBR will aim to achieve at least 6 on-site inspections in 2010 with NBR staff (structural benchmark for end-December 2010), an average of two on-site inspections every four months. This is in addition to continual off-site surveillance for all banks and regular meetings with commercial bank management consistent with the riskbased supervision framework. Over the medium-term, the NBR's compensation scheme and professional development should be enhanced to improve staff motivation and increase staff retention. The NBR will also continue to hold joint bank inspection exercises with senior inspectors from other EAC central banks. The NBR will complete revisions to its loan classification and provisioning manual by June 2010 . Furthermore, supported by the U. S. 
Federal Deposit Insurance Corporation (FDIC), the NBR will embark on a study related to the introduction of a Deposit Insurance Protection Scheme covering the banking and microfinance sector.

50. Further deepening of the financial sector is also expected in 2010. One of the credit cooperatives, CSS-ZIGAMA (Credit and Savings Scheme) will be transformed into a commercial bank in 2010. Further pension reforms are also planned. A legal framework underpinning the establishment of a private pension scheme and personal retirement saving accounts is expected to be officially published before end 2010. Regulations to implement this law were drafted and will be issued soon after the law is enacted.

51. The NBR is embarking on a payment system modernization program. Under this program the core activity for the period 2010-2012 will be the implementation and operation of the Rwanda Integrated Payments Processing System (RIPPS). RIPPS will be composed of the Real Time Gross Settlement System (RTGS), an Automated Clearing House (ACH), and a Central Securities Depository (CSD) in one integrated software and running on the same platform. Once the RIPPS is completed (June 2011), the National Payment Systems (NPS) will conform to international standards including the CPSS Core Principles for Systemically-Important Payment Systems and the CPSS-IOSCO Recommendations for Securities Settlement Systems.

52. RIPPS will significantly enhance efficiency of the payment system. Specifically, it will provide a solid and efficient foundation for the development of a range of innovative payment systems and services in the following ways: accelerate the move towards electronic payment instruments, reduce the systemic importance of cash and checks, support the development of an active market in securities and equities, and support Rwanda's integration into regional economies, particularly the East African Community and COMESA. Between 2010 and 2011 the NBR shall endeavor to do the following:

- Implement and operate the RIPPS (by June 2011);

- Finalize the Electronic Transactions law (by September 2010);

- Finalize the law on the Central Securities Depository (by September 2010);

- Produce guidelines on the cards operations (by December 2010);

- Finalize a regulation on Electronic Funds Transfers and Electronic Money transactions (by December 2010);

- Finalize a regulation governing payment and securities systems (by December 2010);

- Set up an oversight frame work for all Payment Systems (by December 2011). 


\section{THE MEDIUM-TERM STRUCUTRAL REFORM AGENDA}

\section{The government is planning further structural reforms over the medium-term in the following areas.}

\section{Revenue Administration}

54. Further revenue administration measures are planned for the medium-term. Building on the reforms implemented in 2010/11, RRA plans to:

- Introduce $e$ taxes (electronic tax prayer registration, tax filing, reporting and payment systems) at RRA headquarters, and roll out to district and provincial offices;

- Automate the tax audit function by introducing computer-based risk assessments and case selection;

- Automate collection of social security contributions and RAMA

- Evaluate the need for the ASYCUDA++ system in the context of EAC regional integration. The medium-term plan is to automate customs risk management functions that will help in orienting customs verification.

\section{Public Financial Management}

55. We will continue our efforts to improve public financial management over the medium term. The upcoming PEFA assessment will inform our PFM strategy over the medium term. While awaiting this assessment, priorities for the medium term are as follows:

a. Rolling out of IFMIS core modules to all budget agencies;

b. further strengthening the MTEF through end-user sensitization and the undertaking of more PERs so that all sectors are covered over the medium term;

c. continue our ongoing efforts towards fiscal decentralization; and

d. RPPA will provide further training for procurement officers; and

e. The Ministry of Finance will continue to sponsor staff involved in PFM reforms for training as qualified professional accountants (ACCAAssociation of Certified Chartered Accountants);

f. The government will also build capacity to conduct Risk Based Systems Audits, Payroll Audits and Value for Money Analysis and IT (Computer- 
aided audit techniques) audits while piloting the establishment of Audit Committees.

\section{Financial Sector}

56. The central bank supervision action plan targets a full compliance with the 25 Basle core principles (BCPs) as well as standardization of legal and regulatory framework across EAC, both by December 2012. Commercial banks continue to clean up their loan portfolios in order to bring back the level of nonperforming loans within acceptable levels by 2012 - currently defined at around 5 percent (in the immediate post-war period, the rate was in excess of 45 percent). A threshold of 7 percent has been set for 2010. The NBR will harmonize its legal and regulatory framework for the supervision of financial institutions with EAC standards. This harmonization will integrate international best practices, relevant elements of the Basel II framework and the findings of reviews and analysis done since the last global financial crisis.

57. Supervision of MFIs and further financial deepening is on the agenda as well. A number of activities are envisaged to start and strengthen SACCOs with the objective of reaching a goal of 30 percent of the financial inclusion in Rwanda by 2012 from 21 percent cited by the Finscope Rwanda 2008 survey. The MFIs will also benefit from the study to be conducted in 2010 with the assistance of FDIC on the introduction of a Deposit Insurance Protection Scheme covering the banking and microfinance sector. By 2011, the Risk Based Supervision (RBS) will be used in some large MFIs.

\section{Fostering economic integration within the EAC}

58. Graduation to full member of the East African Community (EAC) is expected to lead to increased trade with member countries contributing to an increase in exports and a change in the origin of imports. In the financial sector, joint inspections of cross border financial institutions between EAC Central Banks will continue as provided in the Memorandum of Understanding. In preparation for the move to a monetary union, a council is being established to negotiate macroeconomic convergence criteria for member states.

\section{Export Promotion Strategy}

59. A key priority of the government is to exploit opportunities for higher export revenues. The Rwanda Development Board (RDB) is preparing a draft Comprehensive National Export Diversification Strategy and action plan to help improve exports, which we plan to submit to Cabinet by end-August 2010 (Structural Benchmark). The strategy focuses on increasing the value added of existing export sectors and developing new products and services centered around six clusters: (i) dairy processing; (ii) fruits and vegetables processing; (iii) silk; (iv) specialized tourism (including business tourism); (v) mining services; and (vi) information technologies. The strategy also aims to address bottlenecks to make local products more competitive (both locally and for exports). The action plan 
complements ongoing reforms to improve the business climate and access to credit, as well as ongoing efforts to support companies with export through advocacy and training in management and accounting.

\section{RISKS AND CONTINGENCIES}

60. Key economic and financial downside risks to the program include lower-than-expected exports and government revenues, slower than expected recovery in main trading partner countries, and higher import prices, especially oil. If these risks materialize, the government stands ready to adjust its policies, in close consultation with the IMF staff, to ensure the achievement of a sustainable external and debt position by the end of the program period.

\section{DATA PROVISION AND PROGRAM MONITORING}

\section{Statistics}

61. The NISR has made significant progress in the development of both annual and quarterly national accounts statistics. In September 2009, annual GDP estimates were rebased from 2001 base year to 2006, and new enterprise and household budget surveys were used to revise national accounts from 1998 to 2008. Moreover, quarterly GDP by activity and expenditure were compiled with back series from 2006 to 2009. In March 2010, the NISR released provisional national accounts data for 2009 as well as revision of the data for 2007 and 2008.

62. Notwithstanding the progress to date, the NISR recognizes a number of inherent weaknesses in methodology and compilation of annual and quarterly statistics. Lags in VAT returns and VAT refunds hamper the reliability of using this data as a proxy for production. The use of ex-ante crop assessment reports as a proxy for agricultural production, as well as lack of quarterly BOP, lack of comprehensive annual enterprise surveys, and the existence of a large informal sector further hampers the accuracy of compiling national accounts and projecting GDP growth, which constrains policy-making.

63. Given the importance of agriculture in the economy, the NISR will request TA to revise the methodology for conducting ex-ante and ex-post agricultural crop assessments (structural benchmark, end-Dec 2010). Starting with the 2011 agricultural season (Season A February 2011), the NISR will conduct an ex-post crop assessments for the first time, to ascertain the actual value and production of agricultural output. At the same time, it will continue to use ex-ante crop assessment as a leading indicator of future agricultural production. The NISR will also request TA to design the Living Standards Measurement Questionnaire (structural benchmark, end-September 2010) for the upcoming 2010 household survey. The design of the survey will ensure a better benchmarking of the formal and informal sectors of the economy, which is now derived as a residual. Over the medium term, the NISR intends to conduct an annual survey of all enterprise activities so that 
GDP can be more accurately measured from the production side across all sectors of the economy.

64. In other areas, the NBR intends to request technical assistance and training from the Fund in methodology and compilation of BOP statistics. This will be done to address weaknesses in BOP statistics and measure BOP statistics accurately at quarterly frequencies, which would inform monetary and exchange rate policy decisions.

\section{Program Monitoring}

65. Progress in the implementation of the policies under the PSI will be monitored through assessment criteria (ACs), indicative targets (IT), and structural

benchmarks (SBs). Quantitative targets and structural benchmarks, which are established for end-June 2010 and end-December 2010, are detailed in Tables 2 and 3. The attached Technical Memorandum of Understanding, an integral part of this MEFP, contains definitions and adjustors. Program performance will be assessed in the course of reviews that will take place before end-December 2010 and end-June 2011, respectively.

66. We believe that the policies specified in the MEFP provide a basis for sustaining growth, maintaining low inflation, and alleviating poverty — but we stand ready, in further consultation with the Fund, to take additional measures if required. The government will provide the Fund with the information needed to assess progress in implementing our program as specified in the TMU, and will consult with the Fund staff on any measures that may be appropriate at the initiative of the Government or whenever the Fund requests a consultation. 
Table 1. Rwanda: Quantitative Indicative Targets and Benchmarks for 2009 (Billions of Rwandan francs, unless otherwise indicated)

\begin{tabular}{|c|c|c|c|c|c|}
\hline & \multicolumn{5}{|c|}{2009} \\
\hline & March & June & Sep & $\begin{array}{r}\text { December } \\
\text { (Original prog) }\end{array}$ & $\begin{array}{r}\text { December } \\
\text { (revised) }\end{array}$ \\
\hline \multicolumn{6}{|l|}{ Benchmarks and performance Criteria } \\
\hline \multicolumn{6}{|l|}{ Net foreign assets of the NBR (floor on stock) ${ }^{1}$} \\
\hline Actual (program exchange rate) & 267.0 & 312.6 & 344.6 & & 358.0 \\
\hline Adjusted program & 298.3 & 288.1 & 327.8 & & 334.5 \\
\hline Program & 311.6 & 298.0 & 341.6 & 326.1 & 351.8 \\
\hline \multicolumn{6}{|l|}{ Reserve money (ceiling on stock) ${ }^{2}$} \\
\hline Actual & 112.3 & 107.1 & 112.6 & & 115.1 \\
\hline Adjusted program & 114.8 & 107.1 & 112.6 & & 115.1 \\
\hline Program & 123.2 & 128.3 & 113.3 & 114.0 & 116.3 \\
\hline \multicolumn{6}{|l|}{ Net credit to the government (ceiling on flow) } \\
\hline Actual & 41.0 & 2.5 & -8.4 & & 19.3 \\
\hline Adjusted program & -2.3 & -14.3 & -28.4 & & 8.5 \\
\hline Program & -15.6 & -24.2 & -38.0 & -25.2 & -8.8 \\
\hline \multicolumn{6}{|l|}{ Domestic fiscal balance (floor on flow) } \\
\hline Actual & -47.3 & -76.0 & -130.3 & & -187.6 \\
\hline Adjusted program & -6.5 & -82.1 & -159.6 & & -235.1 \\
\hline Program & -35.8 & -72.2 & -145.8 & -209.3 & -235.1 \\
\hline \multicolumn{6}{|l|}{ Total priority spending (floor on flow) } \\
\hline Actual & 88.7 & 170.6 & 254.7 & & 375.3 \\
\hline Adjusted program & 63.6 & 154.5 & 260.0 & & 375.0 \\
\hline Program & 78.2 & 159.8 & 266.9 & 375.0 & 375.0 \\
\hline \multicolumn{6}{|l|}{ New nonconcessional external debt(Millions of US $\$)^{3}$} \\
\hline Actual & 0.0 & 0.0 & 0.0 & & 13.1 \\
\hline Program & 0.0 & 0.0 & 0.0 & 0.0 & 0.0 \\
\hline \multicolumn{6}{|l|}{ New external payment arrears (ceiling on stock) ${ }^{3,4}$} \\
\hline Actual & 0.0 & 0.0 & 0.0 & 0.0 & 0.0 \\
\hline Program & 0.0 & 0.0 & 0.0 & 0.0 & 0.0 \\
\hline \multicolumn{6}{|l|}{ Short-term external debt (ceiling on stock) ${ }^{5}$} \\
\hline Actual & 0.0 & 0.0 & 0.0 & 0.0 & 0.0 \\
\hline Program & 0.0 & 0.0 & 0.0 & 0.0 & 0.0 \\
\hline \multicolumn{6}{|l|}{ Net accumulation of domestic arrears (ceiling on flow) } \\
\hline Actual & 4.0 & -5.0 & 10.0 & & -10.6 \\
\hline Program & -1.6 & -3.3 & -5.4 & -8.4 & -10.1 \\
\hline \multicolumn{6}{|l|}{ Indicative targets } \\
\hline \multicolumn{6}{|l|}{ Broad money (ceiling on stock) ${ }^{1}$} \\
\hline Actual & 432.3 & 436.8 & 467.3 & & 497.7 \\
\hline Program & 543.1 & 555.4 & 480.1 & 474.9 & 478.4 \\
\hline \multicolumn{6}{|l|}{ Actual } \\
\hline \multicolumn{6}{|c|}{ Net Present Value of the Stock of Outstanding Debt (Millions of US $\$)^{6}$} \\
\hline End-December 2007--Actual & & 240.9 & & 240.9 & 240.9 \\
\hline End-December 2008--Actual & & 331.5 & & 331.5 & 331.5 \\
\hline \multicolumn{6}{|l|}{ End-December 2009--Actual } \\
\hline End-December 2007--Program & & 200.4 & & 200.4 & 200.4 \\
\hline End-December 2008--Program & & 286.4 & & 286.4 & 286.4 \\
\hline End-December 2009--Program & & 413.7 & & 413.7 & 413.7 \\
\hline \multicolumn{6}{|l|}{ Ceiling on stock of domestic debt ${ }^{7}$} \\
\hline Actual & 98.8 & 139.4 & 140.2 & & \\
\hline Program & 183.3 & 168.4 & 102.0 & 100.1 & 100.1 \\
\hline Memorandum items: & 53.4 & 96.4 & 190.4 & & 245.0 \\
\hline Expected general budget support (Millions of US $\$)^{8}$ & 96.2 & 173.7 & 330.1 & 424.7 & 424.7 \\
\hline Received & 19.5 & 155.9 & 263.2 & & 347.0 \\
\hline Of which: budget support grants (received) & 19.5 & 155.9 & 263.2 & & 347.0 \\
\hline Of which: budget support grants & 96.2 & 173.7 & 330.1 & 424.7 & 424.7 \\
\hline Baseline privatization receipts (RwF billion) & 0.0 & 0.0 & 4.1 & 0.0 & 0.0 \\
\hline
\end{tabular}

Sources: Rwandese authorities and IMF staff estimates and projections.

${ }^{1}$ At the program exchange rate of RF555/US\$ through March 2009 and RF576.8 from June 2009 to June 2010.

${ }^{2}$ Targets are calculated as an arithmetic average of the stock of reserve money on the last day of each calendar month in the quarter.

${ }^{3}$ This is a continuous indicative target.

${ }^{4}$ Excludes arrears on obligations that are subject to rescheduling.

${ }^{5}$ Ceiling on the stock of external debt (excluding normal imported-related credits) owed or guaranteed by the central governments, local government, or the NBR with original maturity of up to, and including one year.

${ }^{6}$ Figures indicate the NPV projections based on debt contracted at the test date.

${ }^{7}$ Numbers show end of period stocks.

${ }^{8}$ Excluding external donor financing for demobilization and peacekeeping. 
Table 2. Rwanda: Quantitative Assessment Criteria and Indicative Targets for 2010/11 ${ }^{1}$ (Billions of Rwandan francs, unless otherwise indicated)

\begin{tabular}{|c|c|c|c|c|c|}
\hline & \multicolumn{3}{|c|}{2010} & \multicolumn{2}{|c|}{2011} \\
\hline & June & September & December & March & June \\
\hline \multicolumn{6}{|l|}{ Assessment Criteria $^{2}$} \\
\hline \multicolumn{6}{|c|}{ Net foreign assets of the NBR at program exchange rate (floor on stock) ${ }^{3,4}$} \\
\hline Program & 369.3 & 363.0 & 356.6 & 333.9 & 304.2 \\
\hline \multicolumn{6}{|l|}{ Actual (program exchange rate) } \\
\hline \multicolumn{6}{|l|}{ Reserve money (ceiling on stock) ${ }^{5}$} \\
\hline Program & 122.5 & 127.3 & 130.9 & 132.8 & 134.4 \\
\hline \multicolumn{6}{|l|}{ Actual } \\
\hline \multicolumn{6}{|l|}{ Net domestic financing (ceiling on flow) ${ }^{6}$} \\
\hline Program & -7.5 & 6.3 & -11.2 & 37.7 & 72.5 \\
\hline \multicolumn{6}{|l|}{ Actual } \\
\hline \multirow{2}{*}{\multicolumn{6}{|c|}{$\begin{array}{l}\text { New nonconcessional and government guaranteed external debt (US\$ Millions) } \\
\text { (ceiling on stock) } 7,8\end{array}$}} \\
\hline & & & & & \\
\hline Program & 240.0 & 240.0 & 240.0 & 240.0 & 240.0 \\
\hline \multicolumn{6}{|l|}{ Actual } \\
\hline \multicolumn{6}{|c|}{ External payment arrears (US\$ Millions) (ceiling on stock) ${ }^{8}$} \\
\hline Program & 0.00 & 0.00 & 0.00 & 0.00 & 0.00 \\
\hline \multicolumn{6}{|l|}{ Actual } \\
\hline \multicolumn{6}{|l|}{ Indicative Targets } \\
\hline \multicolumn{6}{|l|}{ Domestic revenue collection (floor on flow) ${ }^{6}$} \\
\hline Program & 197.9 & 311.2 & 426.5 & 554.8 & 677.5 \\
\hline \multicolumn{6}{|l|}{ Actual } \\
\hline \multicolumn{6}{|c|}{ Net accumulation of domestic arrears (ceiling on flow) ${ }^{6}$} \\
\hline Program & -3.0 & -5.0 & -7.0 & -9.0 & -11.0 \\
\hline \multicolumn{6}{|l|}{ Actual } \\
\hline \multicolumn{6}{|c|}{ Consolidated domestic debt of public sector (ceiling on stock, eop) } \\
\hline Program & 211.1 & 214.7 & 187.7 & 212.5 & 217.1 \\
\hline \multicolumn{6}{|l|}{ Actual } \\
\hline \multicolumn{6}{|l|}{ Total priority spending (floor on flow) ${ }^{6}$} \\
\hline Program & 212.0 & 326.0 & 442.0 & 557.0 & 672.1 \\
\hline \multicolumn{6}{|l|}{ Actual } \\
\hline \multicolumn{6}{|l|}{ Memorandum items: } \\
\hline General budget grants (Millions of US\$) ${ }^{6} 9$ & 218.2 & 342.6 & 499.4 & 510.3 & 542.1 \\
\hline
\end{tabular}

Sources: Rwandan authorities; and IMF staff estimates and projections.

${ }^{1}$ All items including adjusters are defined in the attached Technical memorandum of Understanding (TMU).

${ }^{2}$ Test dates for assessment criteria are for end-June and end-December, otherwise indicative targets.

${ }^{3}$ At program exchange rate of RWF 571.24 per US dollar for 2010 and 2011.

${ }^{4}$ This will be adjusted upward (downwards) for any excess (shortfall) of budget grants and loans relative to the program baseline. See TMU for details ${ }^{5}$ Targets are calculated as an arithmetic average of the stock of reserve money at the end of each calendar month in the quarter. program projections are done monthly.

${ }^{6}$ Numbers are cumulative from December 31, 2009.

${ }^{7}$ Cumulative from end-June 2010. The ceiling applies to the duration of the three-year PSI and is tied to two projects as specified in the MEFP. It excludes the IFC SWAP agreement of US $\$ 50$ million as well as $\$ 13.1$ million for purchase of two small airplanes both of which were contracted in 2009.

${ }^{8}$ This is a continuous assessment criterion.

${ }^{9}$ Excluding demobilization and $\mathrm{AU}$ peace keeping operations. 
Table 3. Rwanda: Structural Benchmarks for 2010/11

\begin{tabular}{|c|c|c|}
\hline Measure & Target date & Macroeconomic rationale \\
\hline $\begin{array}{l}\text { PFM } \\
\text { 1. Strengthen the MTEF by } \\
\text { completing PERs for two more } \\
\text { sectors (ICT and transportation). }\end{array}$ & End Mar 2011 & $\begin{array}{l}\text { To improve multi-year fiscal } \\
\text { planning. }\end{array}$ \\
\hline $\begin{array}{l}\text { 2. Begin pilot of core modules of } \\
\text { Smartgov (a component of } \\
\text { IFMIS) in Ministries and } \\
\text { Agencies. }\end{array}$ & End July 2010 & $\begin{array}{l}\text { To help budget preparation, } \\
\text { reporting and execution. }\end{array}$ \\
\hline $\begin{array}{l}\text { 3. Submit to Cabinet for approval } \\
\text { a revised public debt policy and } \\
\text { MTDS. }\end{array}$ & End Dec 2010 & $\begin{array}{l}\text { To assess costs and benefits of } \\
\text { scaled-up nonconcessional } \\
\text { financing and analyze fiscal risks } \\
\text { associated with PPPs. }\end{array}$ \\
\hline $\begin{array}{l}\text { Revenue Administration } \\
\text { 4. Submit to Cabinet for approval } \\
\text { draft legislation to transfer } \\
\text { collection and audit functions of } \\
\text { social security contributions } \\
\text { (Pension and RAMA) to RRA. }\end{array}$ & End Dec 2010 & $\begin{array}{l}\text { To improve compliance and } \\
\text { widen the tax base. }\end{array}$ \\
\hline $\begin{array}{l}\text { 5. Improve risk-based } \\
\text { assessment of ASYCUDA++ by } \\
\text { electronic submission of } \\
\text { supporting customs documents. }\end{array}$ & End June 2010 & $\begin{array}{l}\text { To modernize customs collection } \\
\text { and properly assess liabilities. }\end{array}$ \\
\hline $\begin{array}{l}\text { Financial sector } \\
6 . \text { NBR to conduct minimum of } \\
\text { [6] on-site bank inspections with } \\
\text { NBR staff. }\end{array}$ & End Dec 2010 & $\begin{array}{l}\text { To keep up with both on and off } \\
\text { site prudential oversight of banks } \\
\text { consistent with risk-based } \\
\text { supervision framework. }\end{array}$ \\
\hline $\begin{array}{l}\text { Monetary Policy } \\
\text { 7. NBR to publish (and put on its } \\
\text { website) the underlying } \\
\text { economic assessments } \\
\text { supporting MPC decisions within } \\
\text { one month after every quarterly } \\
\text { MPC meeting. }\end{array}$ & Start date - End June 2010 & $\begin{array}{l}\text { To improve communication and } \\
\text { shape expectations of market } \\
\text { and general public. This would } \\
\text { also enhance effectiveness of the } \\
\text { monetary policy framework. }\end{array}$ \\
\hline $\begin{array}{l}\text { Exchange rate } \\
\text { 8. Announce the introduction of } \\
\text { the interbank exchange rate } \\
\text { corridor framework. }\end{array}$ & End Jun 2010 & $\begin{array}{l}\text { To improve foreign exchange } \\
\text { market efficiency and allow } \\
\text { exchange rates to move in line } \\
\text { with fundamentals. }\end{array}$ \\
\hline $\begin{array}{l}\text { Export sector } \\
\text { 9. Submit to Cabinet for approval } \\
\text { the draft export diversification } \\
\text { strategy and action plan. }\end{array}$ & End Aug 2010 & To broaden the export base. \\
\hline $\begin{array}{l}\text { Statistics } \\
\text { 10. Finalize design of Living } \\
\text { Standards Measurement } \\
\text { Questionnaire (EICV). } \\
\text { 11. Revise methodology for } \\
\text { conducting ex-ante and ex-post } \\
\text { agricultural crop assessments. }\end{array}$ & $\begin{array}{l}\text { End Sep } 2010 \\
\text { End Dec } 2010\end{array}$ & $\begin{array}{l}\text { To improve measurement of } \\
\text { GDP growth } \\
\text { To improve measurement of GDP } \\
\text { growth }\end{array}$ \\
\hline
\end{tabular}




\section{Attachment II. Technical Memorandum of Understanding May 25, 2010}

1. This memorandum defines the quantitative targets described in the memorandum of economic and financial policies (MEFP) for the period June 30, 2010-June 30,2013 supported by the IMF Policy Support Instrument (PSI), and sets out the data reporting requirements.

\section{Quantitative Program Targets}

2. The quantitative program will be assessed through assessment criteria (AC) and indicative targets (IT) for the duration of the program.

3. AC will apply to the following indicators for end-June and end-December (the test dates) throughout the program period; other dates are IT:

- $\quad$ Floor on stock of net foreign assets (NFA) of the National Bank of Rwanda (NBR);

- $\quad$ Ceiling on stock of reserve money;

- $\quad$ Ceiling on flow net domestic financing (NDF) of the central government;

- Ceiling on contracting or guaranteeing of new nonconcessional external debt by the public sector; and

- $\quad$ Ceiling on stock of external payment arrears of the public sector.

4. IT targets apply to the following indicators throughout the program period:

- Floor on flow domestic revenue collection of the central government;

- Ceiling on flow net accumulation of domestic arrears of the central government;

- Ceiling on stock of consolidated domestic debt of the public sector; and

- Floor on flow priority spending.

5. Assessment criteria on contracting or guaranteeing of new nonconcessional external debt by the public sector and stock of external payment arrears of the public sector are applicable on a continuous basis for the duration of the program.

6. Program exchange rates. For accounting purposes, the following program exchange rates which are end-December 2009 rates apply for 2010 and 2011: 


\begin{tabular}{|l|l|}
\hline \multicolumn{2}{|c|}{ Program Exchange Rates (US\$ per currency unit, unless indicated otherwise) } \\
\hline Rwanda Franc (per US\$) & 571.24 \\
\hline Euro & 1.4599 \\
\hline British pound & 1.6241 \\
\hline Japanese Yen (per US\$) & 89.5610 \\
\hline SDR & 1.5822 \\
\hline
\end{tabular}

\section{A. Institutional Coverage of the Fiscal Sector}

7. The central government fiscal operation table comprises the treasury and line ministries, hereafter referred to as the government unless specified otherwise.

\section{B. Targets related to the Execution of the Fiscal Program}

\section{Ceiling on net domestic financing of the government}

8. A ceiling applies to NDF, which is measured cumulatively from December 31, 2009 for June 30, 2010; December 31, 2010; and June 30, 2011. Other dates are indicative targets

9. Definition. NDF of the government is defined as change in the sum of (i) net banking sector credit to the government and (ii) non-bank holdings of the government domestic debt.

10. Net banking sector credit to the government is defined as

- $\quad$ credit to the government from the banking system (as recorded in the monetary survey), including credit to the government, provinces and districts, outstanding consolidated government debt held by the banking system, ${ }^{1}$ government debt o the NBR incurred as a result of the 1995 devaluation (RWF 9 billion), the overdraft to the prewar government (RWF 2 billion); ${ }^{2}$

- minus total government deposits with the banking system (as recorded in the monetary survey), including the main treasury account, line ministries, the fund for

\footnotetext{
${ }^{1}$ Consisting of bank holdings of treasury bills, bonds (domestic), old development bonds (pre-1994 debt), new development bonds (including those used for recapitalization of banks), and other accounts receivables.

${ }^{2}$ The authorities will inform IMF staff of any substantive changes in government accounts with the banking system, which may affect the calculation of net bank claims.
} 
assistance to genocide survivors, the Rwanda Revenue Authority, the electoral commission, the demobilization commission, fonds routier, privatization account, and any other autonomous public enterprises and public agencies over which the government has direct control over their deposits. Thus, this definition excludes any government deposits, over which the central government does not have any direct control (i.e., for project accounts, counterpart funds and fonds publics affectés).

11. Non-bank holdings of the government domestic debt consists of non-bank holdings of treasury bills, bonds (domestic and nonresident), old development bonds (pre-1994 debt), new development bonds (including those used for recapitalization of banks), and other accounts receivables.

\section{Adjusters to the NDF:}

- $\quad$ The ceiling on NDF will be adjusted upward (downward) by the amount of any shortfall (excess) between actual and programmed budgetary grants ${ }^{3}$ (defined in Table 2 of Quantitative AC and IT). This adjustment will be capped at the equivalent of US\$24 million, evaluated in Rwanda francs at the program exchange rate.

- $\quad$ The ceiling on NDF will be adjusted upward by the amount of expenditure for food imports in the case of a food emergency.

13. Reporting requirement. Data on NDF (showing separately treasury bills and government bonds outstanding, other government debt, and central government deposits), each type of debt to be shown by debt holder, and its adjusters will be transmitted on a monthly basis within five weeks from the end of each month. Deposits of the government with the NBR and with the commercial banks will be separated from the deposits of the public enterprises and autonomous public agencies and agencies that the government does not have any direct control over.

\section{Domestic revenues}

14. Definition. The floor on domestic government revenue is defined as total government revenue (tax and non-tax revenues), per the central government fiscal operation table, but excluding external grants, and privatization receipts.

15. Reporting requirement. Detailed data on domestic revenues will be transmitted on a monthly basis within five weeks of the end of each month.

${ }^{3}$ Budgetary grants exclude COMESA grants and HIPC grants. 


\section{Floor on priority expenditure}

16. The floor applies to priority spending of the government which is measured cumulatively from December 31, 2009.

17. Definition. Priority spending is defined as the sum of those recurrent expenditures, domestically-financed capital expenditures, and net lending that the government has identified as priority in line with the EDPRS. Priority expenditures are monitored through the computerized SIBET expenditure management system which tracks priority spending of the annual budget at the program level.

18. Reporting requirement. Data on priority expenditure will be transmitted on a monthly basis within five weeks of the end of each month.

\section{Net accumulation of domestic arrears of the government}

19. A ceiling applies to net accumulation of domestic arrears of the government which is measured cumulatively from December $31,2009 .^{4}$

20. Definition. The net accumulation of arrears is defined as the difference between the gross accumulation of new domestic arrears (measured as the difference between payment orders and actual payments related to payment orders issued) and gross repayment of any arrears outstanding on December 31, 2009 (including repayment of float in 2009 and the repayment of older arrears).

21. Reporting requirement. Data on repayment of domestic arrears and the remaining previous-year stock of arrears will be transmitted on a monthly basis within five weeks of the end of each month.

\section{Limits on External Debt}

\section{Limit on New Nonconcessional External Debt of the Public Sector}

22. A ceiling applies to the contracting and guaranteeing by the public sector of new nonconcessional borrowing debt with nonresidents (see below for the definition of the public sector, concessionality and debt). The ceiling is given in Table 2 of the MEFP which applies continuously from end-June 2010 to end-June 2013; it excludes the swap agreement of US\$50 million signed in November 2009 between the NBR and the IFC and US\$13.1 million for purchase of two air planes in 2009. The ceiling also applies to private debt for which official guarantees have been extended, including future swaps involving foreign currency loans guaranteed by the public sector (see below for swaps), and which, therefore, constitute a contingent liability of the public sector.

\footnotetext{
${ }^{4}$ A negative target thus represents a floor on net repayment.
} 
23. Definition of the public sector. The public sector comprises the general government (the central government, the NBR, local governments which include provinces and districts) and entities in which the government holds a controlling stake - owning more than 50 percent of the shares or the ability to determine general corporate policy. ${ }^{5}$

24. For program purposes, the guarantee of a debt arises from any explicit legal obligation of the public sector to service a debt in the event of nonpayment by the debtor (involving payments in cash or in kind), or from any implicit legal or contractual obligation of the public sector to finance partially or in full any shortfall incurred by the debtor.

25. Definition of concessionality For program purposes, a debt is concessional if it includes a grant element of at least 35 percent, calculated as follows: the grant element of a debt is the difference between the present value (PV) of debt and its nominal value, expressed as a percentage of the nominal value of the debt. The PV of debt at the time of its contracting is calculated by discounting the future stream of payments of debt service due on this debt. ${ }^{6}$ The discount rates used for this purpose are the currency specific commercial interest reference rates (CIRRs), published by the Organization for Economic Cooperation Development (OECD). For debt with a maturity of at least 15 years, the ten-year-average CIRR will be used to calculate the PV of debt and, hence, its grant element. For debt with a maturity of less than 15 years, the six-month average CIRR will be used. To both the ten-year and six-month averages, the same margins for differing repayment periods as those used by the OECD need to be added $(0.75$ percent for repayment periods of less than 15 years, 1 percent for 15 to 19 years, 1.15 percent for 20 to 29 years, and 1.25 percent for 30 years or more).

26. The definition of debt, for the purposes of the limit in the AC, is set out in point 9 of the Guidelines on Performance Criteria with Respect to External Debt (Executive Board's Decision No. 6230-(79/140), as amended by Decision No. 14416-(09/91), effective December 1, 2009). It not only applies to the debt as defined in Point 9 of the Executive Board decision, but also to commitments contracted or guaranteed for which value has not been received. The definition of debt set forth in No. 9 of the Guidelines on Performance Criteria with Respect to External Debt in Fund Arrangements reads as follows:

\footnotetext{
${ }^{5}$ Government control of an entity can be established based on the following criteria: (i) ownership of the majority of the voting interest; (ii) control of the board or other governing body; (iii) control of the appointment and removal of key personnel; (iv) control of key committees of the entity; (v) golden shares and options; (vi) regulation and control; (vii) control by a dominant public sector customer or group of public sector customers; and (viii) control attached to borrowing from the government.

${ }^{6}$ The calculation of concessionality will take into account all aspects of the loan agreement, including maturity, grace period, payment schedule, upfront commissions, and management fees.
} 
(a) The term "debt" will be understood to mean a current, i.e., not contingent, liability, created under a contractual arrangement through the provision of value in the form of assets (including currency) or services, and which requires the obligor to make one or more payments in the form of assets (including currency) or services, at some future point(s) in time; these payments will discharge the principal and/or interest liabilities incurred under the contract. Debts can take a number of forms, the primary ones being as follows:

(i) loans, i.e., advances of money to the obligor by the lender made on the basis of an undertaking that the obligor will repay the funds in the future (including deposits, bonds, debentures, commercial loans and buyers' credits) and temporary exchanges of assets that are equivalent to fully collateralized loans under which the obligor is required to repay the funds, and usually pay interest, by repurchasing the collateral from the buyer in the future (such as repurchase agreements and official swap arrangements);

(ii) suppliers' credits, i.e., contracts where the supplier permits the obligor to defer payments until sometime after the date on which the goods are delivered or services are provided; and

(iii) leases, i.e., arrangements under which property is provided which the lessee has the right to use for one or more specified period(s) of time that are usually shorter than the total expected service life of the property, while the lesser retains the title to the property. The debt is the present value (at the inception of the lease) of all lease payments expected to be made during the period of the agreement excluding those payments that cover the operation, repair, or maintenance of the property.

(b) Under the definition of debt set out in point 9(a) above, arrears, penalties, and judicially awarded damages arising from the failure to make payment under a contractual obligation that constitutes debt. Failure to make payment on an obligation that is not considered debt under this definition (e.g., payment on delivery) will not give rise to debt.

\section{Limit on the stock of external payment arrears}

27. A continuous assessment criterion applies to the non-accumulation of payment arrears on external debt contracted or guaranteed by the public sector. External payment arrears consist of external debt service obligations (principal and interest) that have not been paid at the time they are due, as specified in the contractual agreements, but shall exclude arrears on obligations that are subject to rescheduling.

\section{Consolidated Domestic Debt of the Public Sector (DD)}

28. The ceiling on DD applies to domestic commitments contracted or guaranteed by the public sector. This also applies to private debt for which official guarantees have been 
extended either implicitly or explicitly. The authorities would inform Fund staff of any changes in debt position of public sector entities.

\section{Adjusters:}

- In the case of a shortfall in programmed grants, the ceiling on consolidated domestic debt of public sector will be adjusted upward by the same amount as the increase in the ceiling in the NDF, but capped per paragraph 12 .

- $\quad$ The ceiling on the DD will be adjusted upward by the amount of expenditure for food imports in the case of a food emergency but capped per adjustment to NDF in paragraph 12 .

30. Reporting requirement. Data on domestic debt of the public sector will be transmitted on a monthly basis within five weeks of the end of each month.

\section{Targets for Monetary Aggregates}

\section{Net foreign assets of the National Bank of Rwanda (NFA)}

31. A floor applies to the NFA of the NBR for June 30, 2010, December 31, 2010 and June 30, 2011 targets. Other dates are indicative targets throughout the program.

32. Definition. NFA of the NBR in Rwanda francs are defined, consistent with the definition of the Special Data Dissemination Standard (SDDS) template, as external assets readily available to, or controlled by, the NBR net of its external liabilities. Pledged or otherwise encumbered reserve assets (including swaps) are to be excluded; such assets include, but are not limited to, reserve assets used as collateral or guarantee for third party external liabilities. Foreign assets and foreign liabilities in U.S. dollars are converted to Rwanda francs by using the U.S. dollar/Rwanda franc program exchange rate. Foreign assets and liabilities in other currencies are converted to U.S. dollars by using the actual end-ofperiod U.S. dollar/currency exchange rate. Foreign liabilities include, inter alia, use of IMF resources (CCFF and post-conflict emergency assistance purchases and SAF/ESAF/PRGF disbursements).

\section{Adjusters:}

- $\quad$ The floor on NFA will be adjusted downward by the amount of any shortfall between actual and programmed budgetary grants. This adjustment will be capped at the equivalent of US\$24 million, evaluated in Rwanda francs at the program exchange rate.

- $\quad$ The floor on NFA will be adjusted downward by the amount of expenditure for food imports in the case of a food emergency. 
34. Reporting requirement. Data on foreign assets and foreign liabilities of the NBR will be transmitted on a weekly basis within seven days of the end of each week. This transmission will include daily and weekly data on the NBR's foreign exchange liabilities to commercial banks (including required reserves with the NBR) and the exchange rate used for their conversion into Rwanda francs will be shown separately.

\section{Reserve money}

35. A ceiling applies to the stock of reserve money for the June 30, 2010, December 31, 2010 and June 30, 2011 targets. Other dates are indicative targets throughout the program.

36. The stock of reserve money target for a given quarter will be calculated as the arithmetic average of the stock of reserve money at the end of each calendar month in the quarter. Daily average of reserve money in the last month of each quarter will constitute the actual to be compared with the target.

37. Reserve money is defined as the sum of currency in circulation, commercial banks' reserves, and other nonbank deposits at the NBR.

\section{Adjuster:}

- $\quad$ The ceiling on the stock of reserve money will be adjusted symmetrically for a change in the required reserve ratio of commercial banks. The adjustor will be calculated as (new reserve ratio minus program baseline reserve ratio) multiplied by actual amount of liabilities (Rwanda Franc plus foreign-currency denominated) in commercial banks.

39. Reporting requirement. Data on reserve money will be transmitted on a weekly basis within seven days of the end of each week. This transmission will include a daily and a weekly balance sheet of the NBR which will show all items listed above in the definitions of reserve money.

\section{Other Data Reporting Requirements}

40. For the purposes of program monitoring, the Government of Rwanda will provide the data listed in Table 1 below, weekly data within seven days of the end of each week; monthly data within five weeks of the end of each month; annual data as available.

41. The authorities will inform the IMF staff in writing at least ten business days (excluding legal holidays in Rwanda or in the United States) prior to making any changes in economic and financial policies that could affect the outcome of the financial program. Such policies include but are not limited to customs and tax laws (including tax rates, exemptions, allowances, and thresholds), wage policy, and financial support to public and private enterprises. The authorities will similarly inform the IMF staff of any nonconcessional 
external debt contracted or guaranteed by the government, the NBR, or any statutory bodies, and any accumulation of new external payments arrears on the debt contracted or guaranteed by these entities. The authorities will furnish an official communication to the IMF describing program performance of quantitative and structural assessment criteria and benchmarks within 8 weeks of a test date. The authorities will on a regular basis submit information to IMF staff with the frequency and submission time lag as indicated in Table 1. The information should be mailed electronically to AFRRWA@IMF.ORG . 
Table 1. Summary of Reporting Requirements

\begin{tabular}{|c|c|c|c|}
\hline & $\begin{array}{r}\text { Frequency } \\
\text { of } \\
\text { Data }^{6}\end{array}$ & $\begin{array}{r}\text { Frequency } \\
\text { of } \\
\text { Reporting }^{6}\end{array}$ & $\begin{array}{r}\text { Frequency } \\
\text { of } \\
\text { Publication }^{6}\end{array}$ \\
\hline Exchange Rates & D & W & M \\
\hline $\begin{array}{l}\text { International Reserve Assets and Reserve Liabilities of the } \\
\text { Monetary Authorities }{ }^{1}\end{array}$ & W & W & M \\
\hline Reserve/Base Money & W & W & M \\
\hline Broad Money & $\mathrm{M}$ & M & $\mathrm{M}$ \\
\hline Central Bank Balance Sheet & W & W & M \\
\hline Consolidated Balance Sheet of the Banking System & $\mathrm{M}$ & M & M \\
\hline Interest Rates ${ }^{2}$ & $\mathrm{M}$ & $\mathrm{M}$ & M \\
\hline Consumer Price Index & M & M & M \\
\hline $\begin{array}{c}\text { Revenue, Expenditure, Balance and Composition of Financing }{ }^{3} \\
- \text { - General Government }^{4}\end{array}$ & $\mathrm{M}$ & M & M \\
\hline $\begin{array}{l}\text { Revenue, Expenditure, Balance and Composition of } \\
\text { Financing }{ }^{3}-\text { Central Government }\end{array}$ & M & M & M \\
\hline Stocks of public sector and publically-Guaranteed Debt ${ }^{5}$ & A & A & A \\
\hline External Current Account Balance & A & SA & A \\
\hline Exports and Imports of Goods and Services & A & A & A \\
\hline GDP/GNP & A & SA & A \\
\hline
\end{tabular}

${ }^{1}$ Includes reserve assets pledged or otherwise encumbered as well as net derivative positions.

${ }^{2}$ Both market-based and officially-determined, including discount rates, money market rates, rates on treasury bills, notes and bonds.

${ }^{3}$ Foreign, domestic bank, and domestic nonbank financing.

${ }^{4}$ The general government consists of the central government (budgetary funds, extra budgetary funds, and social security funds) and state and local governments.

${ }^{5}$ Including currency and maturity composition.

${ }^{6}$ Daily (D); Weekly (W); Monthly (M); Quarterly (Q); Annually (A); Semi-annually (SA); Irregular (I). 


\section{INTERNATIONAL MONETARY FUND}

RWANDA

\section{Request for a New Policy Support Instrument_-Informational Annex \\ Prepared by the African Department \\ (In consultation with other departments) \\ Approved by Mark Plant and Christian Mumssen}

May 28, 2010

- $\quad$ Relations with the Fund. Describes financial and technical assistance by the IMF and provides information on the safeguards assessment and exchange system.

- Joint Bank-Fund Work Program. Describes Bank-Fund collaboration through June 2011. 


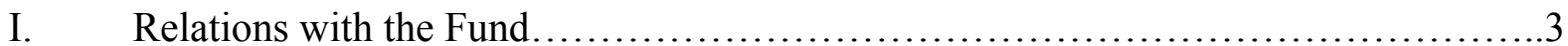

II. Joint Bank-Fund Work Program........................................ 9 


\section{APPENDIX I. RWANDA: RELATIONS WITH THE FUND}

(As of April 30, 2010)

I. Membership Status: Joined: September 30, 1963;

$\underline{\text { Article VIII }}$

II. General Resources Account:

SDR Million

Quota

80.10

\%Quota

Fund holdings of currency

80.11

100.00

$\underline{\text { Reserve Tranche Position }}$

0.00

100.02

Reserve Tranche Position

III. SDR Department:

SDR Million

\%Allocation

Net cumulative allocation

76.82

100.00

Holdings

83.52

108.71

\section{Outstanding Purchases and Loans:}

PRGF Arrangements ${ }^{1}$

SDR Million

\%Quota

9.72

12.14

\section{Latest Financial Arrangements:}

\begin{tabular}{lllrr} 
Type & $\begin{array}{c}\text { Date of } \\
\text { Arrangement }\end{array}$ & $\begin{array}{c}\text { Expiration } \\
\text { Date }\end{array}$ & $\begin{array}{c}\text { Amount Approved } \\
\text { (SDR Million) }\end{array}$ & $\begin{array}{r}\text { Amount Drawn } \\
\text { (SDR Million) }\end{array}$ \\
\hline PRGF & June 12, 2006 & August 07, 20092 & 8.01 & 6.84 \\
PRGF & August 12, 2002 & June 11, 2006 & 4.00 & 4.00 \\
PRGF & June 24, 1998 & April 30, 2002 & 71.40 & 61.88
\end{tabular}

\section{Projected Payments to Fund ${ }^{3}$}

(SDR Million; based on existing use of resources and present holdings of SDRs):

\begin{tabular}{lccccc} 
& \multicolumn{6}{c}{ Forthcoming } \\
\cline { 2 - 6 } & $\underline{2010}$ & $\underline{2011}$ & $\underline{2012}$ & $\underline{2013}$ & $\underline{2014}$ \\
Principal & 0.06 & 0.40 & 0.68 & 1.14 & 1.60 \\
Charges/Interest & $\underline{0.00}$ & $\underline{0.00}$ & $\underline{0.02}$ & $\underline{0.02}$ & $\underline{0.02}$ \\
Total & & $\underline{0.40}$ & $\underline{0.71}$ & $\underline{1.16}$ & $\underline{1.61}$
\end{tabular}

\footnotetext{
${ }^{1}$ Now known as Extended Credit Facility (ECF).

${ }^{2}$ On June 4, the PRGF was extended from June 11, 2009 to August 14, 2009, to allow time for the completion of the sixth and final review, and for making the final disbursement under the PRGF arrangement.

${ }^{3}$ When a member has overdue financial obligations outstanding for more than three months, the amount of such arrears will be shown in this section.
} 
VII. Implementation of HIPC Initiative:

I. Commitment of HIPC assistance

Decision point date

Assistance committed by all creditors

(US\$ million) $^{4}$

Of which: IMF assistance (US\$ million)

(SDR equivalent in millions)

Completion point date
Enhanced

framework

Dec 2000

63.40

46.79

Apr 2005

II. Disbursement of IMF assistance (SDR million)

Assistance disbursed

Interim assistance

Completion point balance

Additional disbursement of interest income ${ }^{5}$

Total disbursements

VIII. Implementation of Multilateral Debt Relief Initiative (MDRI)

I. MDRI -eligible debt (SDR Million) ${ }^{6}$

Financed by: MDRI Trust

Remaining HIPC resources

II. Debt Relief by Facility (SDR million)

\section{Delivery Date}

January 2006
GRA

N/A
PRGT

52.74
Total

52.74

\footnotetext{
${ }^{4}$ Assistance committed under the original framework is expressed in net present value (NPV) terms at the completion point, and assistance committed under the enhanced framework is expressed in NPV terms at the decision point. Hence these two amounts can not be added.

${ }^{5}$ Under the enhanced framework, an additional disbursement is made at the completion point corresponding to interest income earned on the amount committed at the decision point but not disbursed during the interim period.

6 The Multilateral Debt Relief Initiative (MDRI) provides 100 percent debt relief to eligible member countries that are qualified for the assistance. The debt relief covers the full stock of debt owed to the Fund as of end2004 which remains outstanding at the time the member qualifies for such debt relief. The MDRI is financed by bilateral contributions and the Fund's own resources, as well as the resources already disbursed to the member under the HIPC Initiative (see Section VII above).
} 
Decision point - point at which the IMF and the World Bank determine whether a country qualifies for assistance under the HIPC Initiative and decide on the amount of assistance to be committed.

Interim assistance - amount disbursed to a country during the period between decision and completion points, up to 20 percent annually and 60 percent in total of the assistance committed at the decision point (or 25 percent and 75 percent, respectively, in exceptional circumstances).

Completion point - point at which a country receives the remaining balance of its assistance committed at the decision point, together with an additional disbursement of interest income as defined in footnote 4 above. The timing of the completion point is linked to the implementation of pre-agreed key structural reforms (i.e., floating completion point).

\section{Safeguards Assessments:}

Under the Fund's safeguards assessment policy, the National Bank of Rwanda (NBR) was subject to an update assessment with respect to the PRGT arrangement approved on June 5, 2006 (effective June 12, 2006). The assessment's priority recommendations concerned timely publication of the audited financial statements, further improving external audit arrangements, and full implementation of IFRS. An update safeguards assessment is not required in the case of the non-financial arrangement with the Fund such as the PSI, however, such assessment may be voluntary requested by the country.

\section{Exchange System:}

The currency of Rwanda is the Rwandan franc. On December 1998, Rwanda accepted the obligations under Article VIII, Sections 2, 3 and 4 of the IMF and maintains a system free of restrictions on the making of payments and transfers for current international transactions. As of May 14, 2010 the official exchange rate was RWF 579.07 per U.S. dollar. Under the revised classification of the exchange rate arrangements, Rwanda's exchange rate is classified as "stabilized arrangement". The official reference exchange rate is determined daily by the National Bank of Rwanda (NBR) using a 5 day weighted average rate of commercial bank rates and the NBR's rate for the previous day's operations to which a fixed margin of $+/-0.6$ percent is applied to derive the selling and buying rates. ${ }^{7}$ With a view to introducing more flexibility in its exchange rate policy, since March 24, 2010 NBR's intervention in the foreign exchange market is based on interbank rates and at pre-set criteria.

\footnotetext{
${ }^{7}$ From January 16th, 2009 to January 11th, 2010, The NBR's average reference rate (ARR) was calculated as the ten days weighted moving average. With effect from January 12th, 2010, the ARR has chanoed to 5 davs moving average.
} 


\section{Article IV Consultation:}

Rwanda is on the revised 24-month consultation cycle. The Executive Board discussed the staff report for the 2008 Article IV consultation (IMF Country Report No. 09/58) on January 12, 2009.

\section{FSAP Participation, ROSCs, and OFC Assessments:}

A Report on Observance of Standards and Codes on Fiscal Transparency (ROSC) was issued in July 2003. A Financial Sector Assessment Program (FSAP) took place in February 2005. Rwanda has not had an Offshore Financial Center (OFC) assessment.

\section{Technical Assistance and Future Priorities:}

\section{List of Technical Assistance Missions}

2004 AFRITAC East expert on financial regulations.

2004 AFRITAC East mission on treasury reforms.

2004 AFRITAC East expert on implementing cash flow planning and banking arrangements.

2004 FAD tax administration expert on strengthening of revenue administration.

2004 FAD mission on revenue administration.

2004 MFD/LEG mission on Anti-Money Laundering and Combating the Financing of Terrorism.

2004 MFD expert on banking supervision and regulation.

2004 MFD expert on monetary policy, monetary operations, and money markets.

2004 MFD missions on on-site banking supervision.

2005 AFRITAC East expert follow up on cash management.

2005 MFD/LEG mission on Anti-Money Laundering and Combating the Financing of Terrorism.

2005 MFD mission on financial statements of specific bank.

2005 MFD-WB joint FSAP mission.

2005 LEG mission on customs legislation.

2005 STA mission on Balance of Payments statistics.

2005 LEG mission on tax legislation.

2005 FAD mission on public accounting: decentralized accounting for central government.

2005 FAD expert on tax administration.

2005 MFD resident expert on monetary operations, monetary policy, money markets.

2005 MFD expert on banking supervision.

2005 LEG mission on drafting an amendment to the banking law.

2005 MFD mission on banking supervision and bank restructuring.

2006 AFRITAC East experts on establishing intergovernmental fiscal unit in the MOF.

2006 FAD advisor trade facilitation.

2006 MFD FSAP-follow up advisory mission on monetary and foreign exchange operations and NBR internal audit.

2006 LEG mission to strengthen legal and regulatory framework for bank supervision. 
2006 AFRITAC East advisors on finalization of OBL and financial regulations

2007 AFRITAC East advisors on workshops for implementation of OBL and financial regulations.

2007 MCM expert on bank restructuring.

2007 MCM expert on foreign exchange operations.

2008 LEG mission to assist the Ministry of Finance in modernizing the tax system.

2008 LEG mission on banking legislation.

2008 MCM expert on foreign exchange operations.

2008 MCM mission to assist in building up internal audit in the central bank.

2008 FAD expert on revenue administration.

2008 AFRITAC East advisors on (i) improving the budget preparation process and budget accounting, and (ii) changing to the GFS2001- based economic classification (jointly with STA).

2008 MCM mission on monetary policy operations, foreign exchange operations, and assessment of the financial health of banks.

2008 AFRITAC East advisors on enhancement of national accounts estimation.

2009 MCM mission on liquidity management.

2009 MCM mission on bank supervision.

2009 STA mission on rebasing consumer and producer price indices.

$2009 \mathrm{FAD} / \mathrm{AFRITAC}$ East mission to review progress and set the program for TA in budget reform and PFM.

2009 FAD/AFRITAC East mission on customs risk management and valuation.

2009 AFRITAC East advisors on (i) enhancing comprehensiveness and transparency of the fiscal budget and (ii) reform strategy for introduction of program budgeting.

2009 AFRITAC East advisors on quarterly GDP compilation.

2009 AFRITAC East workshop on national accounts.

2009 AFRITAC East advisors on (i) developing a framework for adopting a capital charge for market risk, (ii) enhancements to offsite surveillance system, and (iii) payment system modernization.

2009 AFRITAC East advisors on operational policy development and compliance management in revenue administration.

2009 AFRITAC East workshop on strengthening tax audit and collection enforcement.

2009 FAD/AFRITAC East mission on strengthening fiscal management.

2009 FAD technical assistance evaluation mission.

2009 FAD mission on tax Administration.

2009 FAD/AFRITAC East training in financial programming \& macro-fiscal analysis.

2009 FAD/AFRITAC East mission on bringing external funds on budget.

2009 FAD/AFRITAC East mission on improving budgeting of external funds and expenditure to local government.

2009 FAD/AFRITAC East mission to install expert on revenue administration.

2009 FAD mission on customs business strategy.

2009 FAD mission on customs post-clearance audit training and compliance.

2009 FAD training on tax audit.

2009 FAD mission on customs information technology.

2009 LEG mission on payments and settlements systems law. 
2009 MCM mission on internal audit.

2009 MCM /AFRITAC mission on non bank PSs, stakeholder issues and NPs strategy implementations.

2009 MCM mission on financial stability.

2009 MCM mission on banking supervision.

$2009 \mathrm{MCM} / \mathrm{AFRITAC}$ East training in basic bank supervision skills.

$2009 \mathrm{MCM} / A F R I T A C$ East mission to assist the NBR to assess the current status of Rwanda's banking system.

2009 MCM mission on monetary policy and FOREX.

2009 STA/AFRITAC East mission on improving the PPI.

2009 STA AFRITAC East mission on consumer price statistics.

2009 STA/AFRITAC East mission to assist in launching and facilitating discussions on Rwanda's national accounts compilation.

2009 STA/AFRITAC East mission on quarterly national accounts compilation.

2009 STA/AFRITAC East mission on monetary policy and FOREX.

2010 STA/AFRITAC East mission on national accounts statistics.

2010 LEG mission on payment \& settlement systems.

$2010 \mathrm{MCM} / \mathrm{AFRITAC}$ East mission to develop examination procedures.

2010 STA mission to review data reporting systems.

\section{Future priorities}

The priorities for the Fund's technical assistance in the next year will be in the area of monetary and exchange rate management, supervision of bank and nonbank financial institutions, public finance management and compilation of national account statistics.

\section{Resident Representative:}

Mr. Dmitry Gershenson assumed his duties as Resident Representative in February 2009.

\section{Management Visit:}

The Deputy Managing Director, Mr. Portugal, visited Rwanda during May 3-5, 2007. 


\section{Appendix II. Rwanda: Joint Bank-Fund Work Program}

\begin{tabular}{llll} 
& & VI. & JuNE 2010-JUNE 2011 \\
\hline Title & Products/Activity & $\begin{array}{l}\text { Provisional timing } \\
\text { of mission (if } \\
\text { relevant) }\end{array}$ & $\begin{array}{l}\text { Expected } \\
\text { delivery date }\end{array}$ \\
\hline
\end{tabular}

\section{Mutual Information on Relevant Work Program}

\section{Bank Work \\ Program

\section{A. Strategy and Analytical Work}

Education - Country Status Report

Multi-year Education Policy Analysis

Ongoing

July 2010

Ongoing

Program (FSDP) II (Technical Assistance)

Technical Assistance for Public Expenditure

June 2010

Management in Rwanda

Finance and Private Sector Dialogue

Ongoing

Support to Agriculture Policy Reforms

Ongoing

(Technical Assistance)

Support on Public-Private Partnerships in

June 2010

ICT Sector

Technical Assistance and capacity

July 2010

Building to the VUP

Rwanda Health System Strengthening

July 2010

Social Risk Management of Climate

May 2011

\section{B. Ongoing and New Projects}

Decentralization and Community Development

June 2010

Public Sector Capacity Building Project

Ongoing

Second Rural Sector Support Project

Ongoing

Second Emergency Demobilization and Reintegration Project

Ongoing

Land Husbandry, Water harvesting and Hillside Irrigation

Rwanda Electricity Access Scale-up and Sector-wide approach Project

Ongoing

Ongoing

Ongoing

Sustainable Energy Development Project (GEF)

Ongoing

Integrated Management of Critical Ecosystem Project (GEF)

Second Community Living Standards Grant

Ongoing

Ongoing

Poverty Reduction Support Grant -VI

Ongoing

E-Rwanda

Ongoing 


\begin{tabular}{|c|c|c|c|}
\hline Title & Products/Activity & $\begin{array}{l}\text { Provisional timing } \\
\text { of mission (if } \\
\text { relevant) }\end{array}$ & $\begin{array}{l}\text { Expected } \\
\text { delivery date }\end{array}$ \\
\hline & Transport Sector Development & & March 2011 \\
\hline & Skill Development Project & & February 2011 \\
\hline & Competitiveness and Enterprise Project & & July 2011 \\
\hline \multirow[t]{13}{*}{$\begin{array}{l}\text { IMF Work } \\
\text { Program }\end{array}$} & \multicolumn{3}{|c|}{ A. Missions } \\
\hline & $\begin{array}{l}\text { First Review Under the Policy Support } \\
\text { Instrument }\end{array}$ & September 2010 & November 2010 \\
\hline & \multicolumn{3}{|c|}{ B. Analytical Work. } \\
\hline & $\begin{array}{l}\text { Some Considerations in the Design of a } \\
\text { Liquidity Supervision Framework for } \\
\text { Banks in Rwanda }\end{array}$ & & September 2010 \\
\hline & $\begin{array}{l}\text { Understanding the Exchange Rate Pass- } \\
\text { Through to Domestic Prices in the EAC } \\
\text { Region: Implications for Prospects of } \\
\text { Monetary Integration. }\end{array}$ & & December 2010 \\
\hline & $\begin{array}{l}\text { Estimating the behavioral Equilibrium } \\
\text { Exchange Rate for the EAC Countries }\end{array}$ & & December 2010 \\
\hline & $\begin{array}{l}\text { Monetary Policy Transmission in EAC } \\
\text { Countries }\end{array}$ & & December 2010 \\
\hline & \multicolumn{3}{|c|}{ C. Technical Assistance } \\
\hline & $\begin{array}{l}\text { Fiscal: Tax and customs administration; } \\
\text { revenue forecasting and PFM }\end{array}$ & & \\
\hline & $\begin{array}{l}\text { Monetary: Monetary policy operations; bank } \\
\text { liquidity; interbank markets in local currency } \\
\text { and foreign exchange }\end{array}$ & & \\
\hline & Financial: Bank supervision & & \\
\hline & $\begin{array}{l}\text { Statistics: National accounts and balance of } \\
\text { payments }\end{array}$ & & \\
\hline & \multicolumn{2}{|c|}{ II. Request for Work Program Inputs } & \\
\hline $\begin{array}{l}\text { Bank } \\
\text { Request to }\end{array}$ & $\begin{array}{l}\text { Impact of the global economic crisis on key } \\
\text { macro indicators and growth in Rwanda }\end{array}$ & & Ongoing \\
\hline Fund & Sharing macro-framework updates & & As needed \\
\hline
\end{tabular}




\begin{tabular}{llll}
\hline Title & Products/Activity & $\begin{array}{l}\text { Provisional timing } \\
\text { of mission (if } \\
\text { relevant) }\end{array}$ & $\begin{array}{l}\text { Expected } \\
\text { delivery date }\end{array}$ \\
\hline $\begin{array}{l}\text { Fund } \\
\text { Request to } \\
\text { Bank }\end{array}$ & $\begin{array}{l}\text { Assessment of key infrastructure projects } \\
\text { undertaken by the government. }\end{array}$ & As needed \\
& $\begin{array}{l}\text { Assessment of the PFM and public service } \\
\text { reform program. }\end{array}$ & As needed \\
\hline
\end{tabular}

\section{Agreement on Joint Products}

\begin{tabular}{lll}
\hline Joint & Public and External Debt Sustainability & June 2010 \\
products & $\begin{array}{l}\text { Analysis. } \\
\text { Collaborate together with other development } \\
\text { partners on the annual review of general } \\
\text { budget support. }\end{array}$ & Ongoing \\
& \\
Collaborate on PFM reform program. & \\
& $\begin{array}{l}\text { Collaborate on financial sector reform } \\
\text { program. }\end{array}$ & Ongoing \\
& Ongoing \\
\hline
\end{tabular}




\title{
INTERNATIONAL DEVELOPMENT ASSOCIATION AND INTERNATIONAL MONETARY FUND
}

\author{
RWANDA \\ Joint World Bank/IMF Debt Sustainability Analysis \\ Prepared by staffs of the International Development Association and \\ International Monetary Fund \\ Approved by Sudharshan Gooptu and Sudhir Shetty (IDA) \\ and Mark Plant and Christian Mumssen (IMF)
}

May 28, 2010

Rwanda continues to be assessed as a moderate risk of external debt distress-unchanged

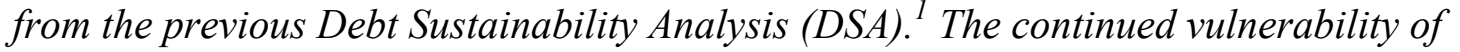
$R$ wanda's debt indicators to an export shock highlights the urgent need to expand the export base. The DSA suggests that there is room for some nonconcessional borrowing - to meet the country's large infrastructure investment needs-without unduly increasing Rwanda's risk of debt distress. Rwanda has a medium-term debt and Public Financial Management (PFM) strategy, conducts its own DSAs, and has some experience with nonconcessional borrowing. Nevertheless, careful vetting, prioritization, and sequencing of the implementation of investment projects by the Rwandan authorities, as well as strengthening implementation capacity, will be essential to maintain debt sustainability over the near and medium term.

\section{BACKGROUND}

1. Rwanda's external debt of the central government (including guaranteed) at end-2009 was US\$749.1 million (14.4 percent of GDP) (Table 1). More than four-fifths of external central government debt is owed to multilateral creditors. External debt has declined from 85 percent of GDP in 2000-04 to about 15 percent of GDP since 2006, thanks to substantial debt relief. Rwanda reached the HIPC Completion Point in April 2005 and also benefited from MDRI relief in January 2006. Domestic debt was RWf 228.3 billion (7.7 percent of GDP or about a third of total public debt at end-2009, down from 13 percent of GDP in 2005-07. Within domestic debt, the end-2009 stock of short-term debt (Treasury bills and central bank monetary instruments) was equivalent to 3.1 percent of GDP.

\footnotetext{
${ }^{1}$ Based on the joint Low-Income Country Debt Sustainability Framework prepared by the IMF and World Bank staff in consultation with the authorities. This DSA replaces the one prepared at the time of the 2008 Article IV Consultation (IMF Country Report No. 09/58) and the update done at the time of the $6^{\text {th }}$ PRGF (now known as Extended Credit Facility (ECF)) Review (Box 2 in IMF Country Report No. 09/264). The fiscal year for Rwanda is July-June; however, the DSA has been produced on a calendar year basis.
} 


\begin{tabular}{|lrrr|}
\hline \multicolumn{4}{|c|}{ Table 1. Rwanda: Composition of Public Debt, end 2009 } \\
\hline & $\begin{array}{r}\text { Millions } \\
\text { of US\$ }\end{array}$ & $\begin{array}{r}\text { Percent } \\
\text { of Total }\end{array}$ & $\begin{array}{r}\text { Percent } \\
\text { of GDP }\end{array}$ \\
\hline Total (External + Domestic) & $\mathbf{1 , 1 4 9}$ & $\mathbf{1 0 0 . 0}$ & $\mathbf{2 2 . 1}$ \\
External Debt & $\mathbf{7 4 9}$ & $\mathbf{6 5 . 2}$ & $\mathbf{1 4 . 4}$ \\
Central Government & 736 & 64.1 & 14.2 \\
Multilateral & 612 & 53.3 & 11.8 \\
IMF & 15 & 1.3 & 0.3 \\
IDA & 257 & 22.3 & 4.9 \\
African Development Bank Group & 155 & 13.5 & 3.0 \\
Other Multilateral & 185 & 16.1 & 3.6 \\
Official Bilateral & 124 & 10.8 & 2.4 \\
Paris Club & 21 & 1.8 & 0.4 \\
Non-Paris Club & 104 & 9.0 & 2.0 \\
Guaranteed by the Central Government & 13 & 1.1 & 0.3 \\
Domestic Debt & $\mathbf{4 0 0}$ & $\mathbf{3 4 . 8}$ & $\mathbf{7 . 7}$ \\
In RWf billions & 228 & 34.8 & 7.7 \\
Of which: Treasury Bills \& Monetary Instruments & 93 & 14.1 & 3.1 \\
\hline Source: Rwandan authorities, IMF and World Bank staff calculations. & & \\
\hline
\end{tabular}

\section{UNDERLYING DSA ASSUMPTIONS}

\section{A. Macroeconomic Assumptions}

2. Real GDP is projected to rebound from the growth slowdown in 2009 to reach 6.9 percent in 2013 before settling at 6.5 percent over the longer term (Table 2).

Projected growth, while above the SSA average, is somewhat conservative when compared to Rwanda's observed growth rates of the past decade (average annual real GDP growth was 8.3 percent in 2000-08). Growth is envisaged to be stimulated by infrastructure investment, the improving business environment, and a positive impulse from regional integration.

Growth in the GDP deflator would gradually decline to 5 percent over the long term, in line with inflation.

3. The primary fiscal balance (excluding grants) is projected to steadily improve mainly on account of stronger revenue collection, capturing gains from the broadening tax base and increasing efficiency of tax administration. Revenues would increase by 2 percentage points of GDP over 2010-15, to 14.8 percent of GDP, and continue to improve modestly thereafter. Primary current expenditures would settle at 15 percent of GDP over the long term, similar to 2010-15. Capital expenditures, on the other hand, would gradually be reduced to almost 8 percent of GDP over the long term as scaled-up spending, including on large infrastructure projects ( $\mid 6-9)$, gradually unwinds. External grants, which have been scaled up in the past few years to help Rwanda cope with the effects of adverse external 
shocks (such as the food and fuel crises) would peak in 2010 at 13.5 percent of GDP and gradually decline, reflecting both a gradual return to normalcy in the medium term and reducing aid dependency over the longer term.

\begin{tabular}{|c|c|c|c|c|c|c|c|c|c|c|}
\hline & 2009 & 2010 & 2011 & 2012 & 2013 & 2014 & 2015 & 2020 & 2025 & 2030 \\
\hline & \multicolumn{10}{|c|}{ (In percent of GDP, unless otherwise indicated) } \\
\hline Nominal GDP (RWf billions) & 2,964 & 3,333 & 3,746 & 4,210 & 4,725 & 5,285 & 5,912 & 10,339 & 18,078 & 31,612 \\
\hline Real GDP (percentage change) & 4.1 & 5.4 & 5.9 & 6.4 & 6.9 & 6.5 & 6.5 & 6.5 & 6.5 & 6.5 \\
\hline GDP Deflator (percentage change) & 11.0 & 6.7 & 6.1 & 5.6 & 5.0 & 5.0 & 5.0 & 5.0 & 5.0 & 5.0 \\
\hline \multicolumn{11}{|l|}{ Fiscal (central government) } \\
\hline External Grants (incl. HIPC Relief) & 11.7 & 13.5 & 11.3 & 10.7 & 9.5 & 8.7 & 7.8 & 5.2 & 4.7 & 4.5 \\
\hline Revenue (excl. External Grants) & 12.8 & 12.8 & 13.3 & 13.8 & 14.2 & 14.6 & 14.8 & 15.5 & 16.1 & 16.7 \\
\hline Revenue (incl. external grants) & 24.6 & 26.3 & 24.6 & 24.5 & 23.8 & 23.3 & 22.7 & 20.7 & 20.8 & 21.2 \\
\hline Primary Expenditures & 25.1 & 26.9 & 25.7 & 25.1 & 23.9 & 23.4 & 22.7 & 22.9 & 22.9 & 22.9 \\
\hline Primary Current Expenditures & 13.9 & 14.9 & 14.2 & 15.1 & 14.9 & 15.1 & 14.8 & 15.0 & 15.0 & 15.0 \\
\hline Capital Expenditure and Net Lending & 11.1 & 12.0 & 11.5 & 10.0 & 9.0 & 8.3 & 7.9 & 7.9 & 7.9 & 7.9 \\
\hline Primary Balance, incl. External Grants & -0.5 & -0.6 & -1.1 & -0.6 & -0.1 & -0.1 & 0.0 & -2.2 & -2.1 & -1.7 \\
\hline Primary Balance, excl. External Grants & -12.2 & -14.1 & -12.4 & -11.3 & -9.6 & -8.8 & -7.8 & -7.4 & -6.8 & -6.2 \\
\hline Net Domestic Financing & -0.8 & -0.3 & 0.0 & 0.0 & 0.0 & 0.0 & 0.0 & 0.0 & 0.0 & 0.0 \\
\hline Interest Rate (percent) & & 0.0 & 0.0 & 0.0 & 0.0 & 8.0 & 8.0 & 8.0 & 8.0 & 8.0 \\
\hline New External Borrowing ${ }^{1}$ & & 3.1 & 4.5 & 1.9 & 1.1 & 0.9 & 0.8 & 2.7 & 2.7 & 2.4 \\
\hline Grant Element of New External Borrowing (percent) & & 12.3 & 6.8 & 23.5 & 43.0 & 42.7 & 42.3 & 40.5 & 39.2 & 37.9 \\
\hline \multicolumn{11}{|l|}{ Balance of Payments } \\
\hline Exports of Goods and Services & 10.2 & 10.9 & 11.4 & 11.8 & 12.3 & 12.8 & 13.4 & 14.9 & 16.4 & 17.9 \\
\hline Imports of Goods and Services & 28.3 & 30.6 & 30.2 & 26.7 & 25.3 & 24.6 & 24.4 & 24.1 & 23.9 & 23.6 \\
\hline Current Account, incl. Official Transfers & -7.3 & -7.9 & -8.8 & -5.4 & -4.4 & -3.7 & -3.5 & -4.2 & -2.4 & -0.4 \\
\hline Foreign Direct Investment & 2.3 & 1.8 & 1.4 & 1.6 & 1.8 & 1.8 & 1.9 & 2.4 & 2.4 & 2.4 \\
\hline Gross Oficial Reserves (months of imports of G\&S) & 5.1 & 4.8 & 4.7 & 4.7 & 4.4 & 4.3 & 4.2 & 4.2 & 4.2 & 4.2 \\
\hline
\end{tabular}

4. As for the financing side of the fiscal deficit, the baseline assumes the government's policy of no new net domestic financing after 2010. Maturing domestic debt is rolled over at an interest rate of 8 percent. Baseline new external borrowing during 2010-12 assumes disbursements from loans that have already been signed, as well as new nonconcessional loan guarantees to finance strategic investment projects (see below for details). The latter explain much of the lower projected grant element of new external borrowing in those years. After 2013, new external borrowing is expected to come largely on concessional terms. Initially about 75 percent of central government external borrowing would be on terms similar to those from IDA, another 5 percent would be from Paris Club bilateral creditors, and the rest (20 percent) from non-Paris Club bilateral creditors. Over time the average terms of the external financing mix are expected to become less favorable, thus implying a falling grant element (from about 43 percent in 2013 to less than 38 percent over the longer term). This pattern for the external borrowing mix differs from the previous DSA update which assumed 100 percent borrowing on terms similar to those of IDA. 
5. On the external front Rwanda is expected to become more open as regional integration continues (opening up regional markets further and reducing barriers to trade for the landlocked country), the business climate improves further, and export sectorsespecially from agriculture, minerals, and tourism - develop. The authorities are developing and implementing policies to lift their export base (see Box 1). Exports of goods and services are projected to increase by 2.5 percent of GDP in 2010-15, and to rise further to nearly 18 percent of GDP in the long term. Imports are expected to settle around 24 percent of GDP in the long term; the erratic pattern of imports in the medium term is due to the lumpiness of imports of capital goods associated with large infrastructure investment projects. The current account deficit is projected to narrow gradually and would be financed in part by higher foreign direct investment (which would reach about 2.4 percent of GDP over the longer term), as the private sector steadily steps up its share in overall investment, including possibly through public-private partnerships.

\section{Box 1. Rwanda: Export Promotion and Diversification}

A key priority of the government is to exploit opportunities for higher export revenues. The Rwanda Development Board (RDB) is preparing a comprehensive national export strategy and action plan to help improve exports. The strategy focuses on increasing the value added of existing export sectors and developing new products and services centered around six clusters: (i) dairy processing; (ii) fruits and vegetables processing; (iii) silk; (iv) specialized tourism (including business travelers); (v) mining services; and (vi) information technologies. The strategy also addresses bottlenecks to make local products more competitive (both locally and for exports). The action plan will expand and coordinate efforts to support companies that export, through advocacy and training in management and accounting, and complements ongoing reforms to improve the business climate and access to credit. The authorities are also looking to hire an international consultant to help develop a master plan for export opportunities in the agribusiness and agro-processing sector, especially to regional markets.

A new Mining Policy (adopted in September 2009) is being implemented. The mining policy is based on five strategic pillars (including strengthening the regulatory environment, improving productivity, and improving value added) and a three-year action plan. The policy aims to: (i) increase mineral exports by 250 percent from \$US 38 million in 2005 to \$US 106 million by 2012; and (ii) increase employment in the sector from 25,000 to 37,000 over the same period. The authorities are also looking to restart the tin smelter, although reliable electricity supply would need to be secured first. Opportunities for product diversification are also being considered, notably for construction materials, energy substances, and precious stones.

\section{B. Large Infrastructure Projects}

6. The Rwandan authorities have an ambitious investment strategy, comprising six strategic, complementary projects which aim to alleviate critical infrastructure constraints to increasing and diversifying exports of goods and services and developing the country into a knowledge-based service economy (Box 2). These investments have the potential for spillovers to employment generation and poverty reduction, and, through their efforts to crowd in foreign investors, the authorities intend to protect priority spending in their budget. The authorities believe that the projects are expected to yield high net economic benefits. 


\section{Box 2. Rwanda: Strategic Investment Projects}

Energy rollout: increase household grid connections from 6 percent at end-2008 to 16 percent by 2013, reach all public health centers and administrative offices, and connect half of all schools (per Economic Development and Poverty Reduction Strategy (EDPRS) objectives). The 2009-13 capital budget is estimated at US\$377 million and would be fully financed with concessional resources.

Core ICT infrastructure: create infrastructure for high-speed broadband by: (i) building high-capacity fiber optic lines by May 2011 ("backbone" project); (ii) providing broadband wireless technology to up to 100,000 users by mid-2010 (Wibro/Wimax project); and (iii) connecting Rwanda to undersea fiber-optic cables by 2013, which could reduce broadband purchase costs by 90 percent. The total cost to the budget is estimated at US\$107 million and financed with concessional resources.

Regional railway: rehabilitate/build railways linking Rwanda to Burundi and Tanzania. A Public Private Partnership (PPP) is being considered to overcome high investment costs and risks (alternative design standards are being considered which could reduce initial cost estimates (US $\$ 4.7$ billion) by 25 percent. The Rwandan authorities plan to detail the public financing requirements for the project once the PPP framework has been designed.

Bugesera Airport: construct a new airport conforming to ICAO standards with sufficient capacity to meet future demand from high-value agro exports and business tourism, and make Rwanda into Central African hub/gateway. The first four-year phase (US\$325 million) would be financed through a PPP.

RwandAir expansion: create strong air links to Rwanda to support service sectors and high-value exports and capture a share of the increasing regional and international air traffic. New management at RwandAir (which is 100 percent publicly owned) developed a business plan for 2010-14 with a US\$116.3 million capital budget. The company purchased two short-range planes (US\$14.5 million) in 2009, and a purchase agreement was signed in December 2009 (US\$85 million) for two mid-range Boeing planes to be delivered in 2011. The government is considering guaranteeing nonconcessional external loans for the purchase of the mid-range planes. The government's objective is to transform RwandAir into a viable business by end-2014 and is committed to its divestment and privatization in the long term.

Kigali Convention Complex: develop business tourism by building a five-star hotel and convention center. Construction started in mid-2009 and is expected to be completed by end-2011 at a cost of US\$300 million. The development company (75 percent publicly owned) received a US\$67 million government loan in 2009 and is negotiating a loan/equity mix with private investors, which would possibly include external financing of up to US\$180.5 million.

7. The six projects are at different stages of implementation. The ICT core infrastructure project is well advanced and the energy rollout project is also progressing, though its finalization may extend beyond 2012. Construction on the Kigali Convention Complex has started and RwandAir has already acquired two small planes which it is using to serve short routes. The regional railway and Bugesera airport projects, on the other hand, are in the design phase — only feasibility studies have been budgeted for so far.

8. The baseline DSA incorporates financing for four of the six strategic investment projects, of which two are on nonconcessional terms - the Kigali Convention Complex and RwandAir. The core ICT infrastructure is fully financed from concessional sources. The electricity rollout project is also being financed from concessional sources; about 40 percent of the full cost of the project has been secured and the remainder of the project will be 
implemented as and when the remaining funding is secured. As for the remaining two projects, Bugesera Airport and regional railroad, the allocation in the 2010/11 budget amounts to less than US\$5 million which is intended to finance feasibility studies. These two projects will be included in future DSAs once the details on the required investment envelope and the type of financing are more concrete. The authorities are considering the use of private public partnerships for these investments to ease pressure on government financing and are strengthening the regulatory framework, which would also enable them to better assess potential contingent liabilities.

9. The baseline DSA assumes US\$240 million in new external loan guarantees for Kigali Convention Complex (US\$180.5 million) and RwandAir (US\$59.5 million). ${ }^{2}$ The government is seeking international participation to secure full funding for the Kigali Convention Complex, which may require government guarantees for external borrowing. The government considers these loan guarantees may be critical to jump start the strategic investment projects and crowd in foreign investors, given a shallow domestic capital market, limited availability of concessional financing, and the need to avoid crowding out the private sector. $^{3}$

\section{EXTERNAL DSA}

\section{A. Policy-Dependent Indicative Thresholds}

10. The Debt Sustainability Framework defines policy-dependent indicative thresholds against which the external debt sustainability indicators are measured. These are based on a country's score on the World Bank's Country Policy and Institutional Assessment (CPIA). Rwanda's CPIA score was 3.68 in 2008 and 3.66 on average over 2006-08, putting it in the "Medium" performance category (the category that corresponds to a three-year average CPIA score between 3.25 and 3.75). For a "Medium" performer like Rwanda the policydependent indicative thresholds are those in Table 3.

\footnotetext{
2 The assumed terms for the external loans that would be guaranteed are as follows: (i) Kigali Convention Complex: ten year maturity with one-year grace and interest rate of 8 percent; and (ii) RwandAir: maturity of ten years and interest rate of 6.75 percent. The bulk of the disbursements of the loans would be in 2011 (for the Kigali Convention Complex, some disbursements would be made in 2010 and 2012 - in line with the rhythm of project execution). The baseline DSA also includes nonconcessional borrowing already contracted in 2009, specifically a loan contracted in March 2009 with Export-Import Bank of China to finance a road project, in an amount of 219 million Chinese yuan (about US\$32 million), and a US\$13.1 million nonconcessional loan guarantee for RwandAir for the purchase of two small planes which were delivered at end-2009.

${ }^{3}$ The feasibility study for the Kigali Convention Complex, prepared by an international consulting firm, found a rate of return of about 13.2 percent. The return on equity for RwandAir has been estimated at 11 percent (by 2014), based on the business plan.
} 


\begin{tabular}{|c|c|c|c|c|}
\hline \multicolumn{3}{|c|}{ Present Value of Debt in Percent of: } & \multicolumn{2}{|c|}{ Debt Service in Percent of: } \\
\hline Exports & GDP & Revenue & Exports & Revenue \\
\hline 150 & 40 & 250 & 20 & 30 \\
\hline
\end{tabular}

\section{B. Baseline Results of the External DSA}

11. The external DSA results indicate that Rwanda's debt dynamics would be sustainable even if the assumed amount of nonconcessional external borrowing is contracted, while the stress tests confirm Rwanda's vulnerability arising from its low export base-unchanged from the previous DSA. Specifically:

- Under the baseline assumptions, all indicators of public and publicly guaranteed external debt stay well below their respective thresholds (Appendix Table 1, Appendix Figure 1).

- The indicative threshold for the ratio of present value (PV) of debt to exports is breached through 2015 when the standard bounds test for exports is applied (test B2 in Appendix Table 2). In addition, the "combination bounds test" breaches the threshold for the PV of debt-to-exports ratio through 2013 (test B5 in Appendix Table 2). ${ }^{5}$

12. While the long-run results of the baseline external DSA are similar to those of the previous DSA update (Figure 1), a few factors appear to explain the observed difference with the previous DSA update in the short run (Table 4). First, the current DSA uses a discount rate of 4 percent, compared to 5 percent in the previous update. The one-percentage point reduction in the discount rate increases the PV of debt in 2011 by some US\$78 million (1.3 percent of GDP). In addition, the additional nonconcessional borrowing adversely affects the sustainability ratios (including the debt service ratios). Finally, additional grants in 2010-11 are mitigating the impact on debt dynamics of the fiscal primary balance (even though projections for the primary balance excluding grants are lower than in the previous DSA). On balance, debt sustainability indicators in the short-to-medium term are projected to exceed those projected in the previous DSA. Nevertheless, the ratios are projected to decline rapidly over the medium term once the additional nonconcessional borrowing ceases and the lower projected primary balance including grants work in favor of the debt dynamics, especially because of higher projected grants over the medium term.

4 The standard bounds test B2 for exports imposes a value growth for exports equal to the historical average minus one standard deviation in 2011-12. Afterwards, export growth returns to the assumed value growth path, implying permanently lower values.

5 The standard "combination bounds test" B5 takes for 2011-12 a one-half standard deviation adverse shock to the historical averages of (i) real GDP growth; (ii) export value growth; (iii) U.S. GDP deflator; and (iv) nondebt creating flows. 
Figure 1. External Debt Sustainability Indicators, 2009-30

(In percent)

Striped: Baseline DSA results from 6th ECF (formerly PRGF) Review. Solid: DSA update.
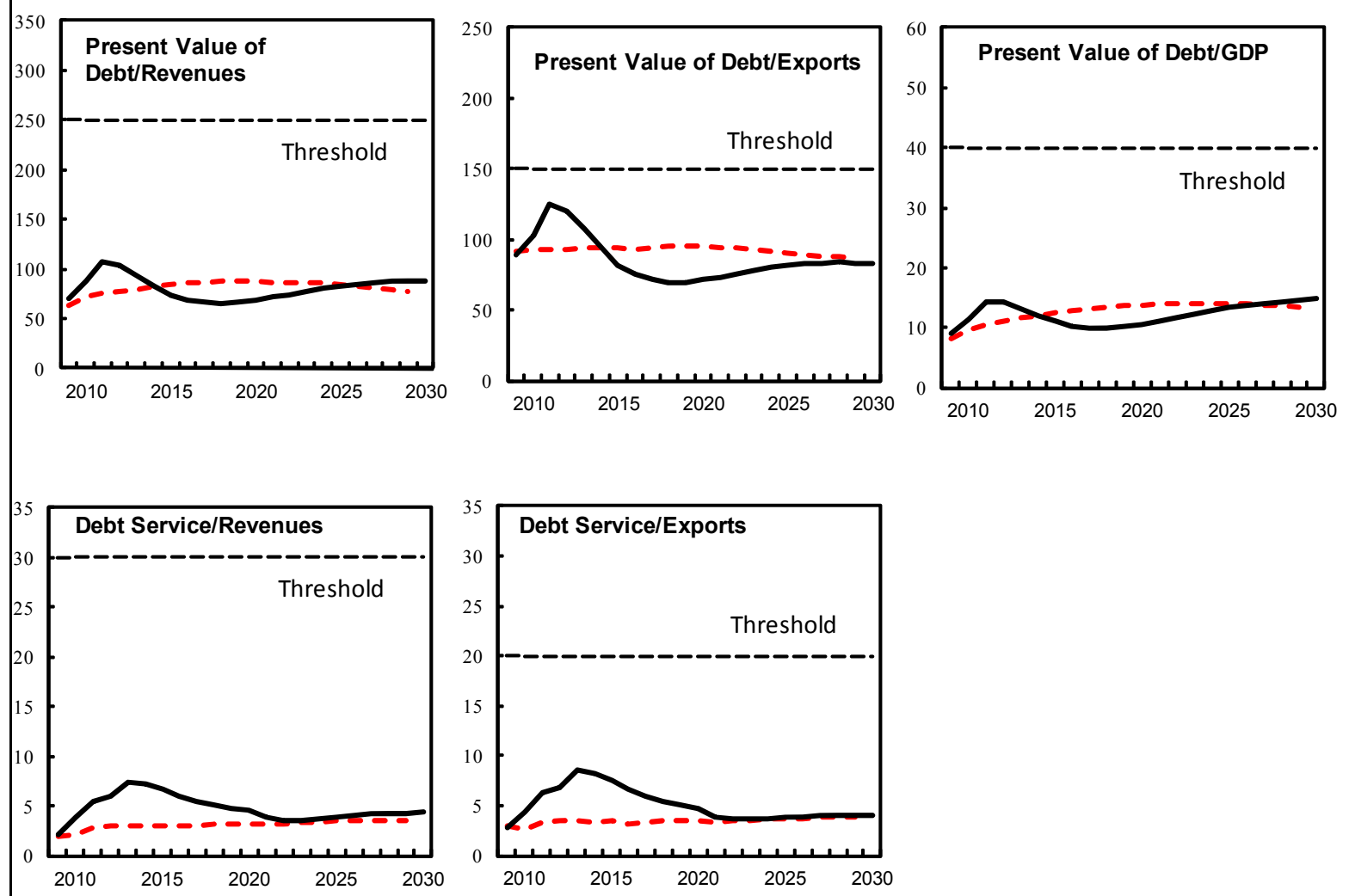

Source: Rwandan authorities, and IMF-World Bank staff estimates and projections. 


\begin{tabular}{|c|c|c|c|c|c|c|c|c|c|}
\hline & \multicolumn{3}{|c|}{ Previous DSA Update $^{1}$} & \multicolumn{3}{|c|}{ DSA Update } & \multicolumn{3}{|c|}{ DSA Update (5\% Discount Rate) } \\
\hline & 2009 & 2010 & $\overline{2011}$ & 2009 & 2010 & $\overline{2011}$ & 2009 & 2010 & 2011 \\
\hline & Proj. & Proj. & Proj. & Est. & Proj. & Proj. & Est. & Proj. & Proj. \\
\hline \multicolumn{10}{|l|}{ External Debt Stock } \\
\hline In millions of U.S. dollars & 837 & 1,015 & 1,173 & 749 & 913 & 1,158 & & & \\
\hline In percent of GDP & 17.2 & 19.6 & 20.9 & 14.4 & 16.4 & 19.3 & & & \\
\hline In millions of U.S. dollars & 414 & 512 & 589 & 470 & 623 & 861 & 416 & 560 & 782 \\
\hline In percent of GDP & 8.3 & 9.7 & 10.4 & 9.1 & 11.2 & 14.3 & 8.0 & 10.1 & 13.0 \\
\hline PV of External Debt to Revenues (percent) & $63.0^{\mathrm{L}}$ & 72.6 & 75.5 & 70.6 & 87.7 & 108.0 & 62.5 & 78.7 & 98.2 \\
\hline PV of External Debt to Exports (percent) & 91.9 & 93.3 & 92.4 & 89.0 & 102.8 & 125.2 & 78.9 & 92.3 & 113.8 \\
\hline External Debt Service to Revenues (percent) & 2.0 & 2.1 & 2.7 & 2.2 & 3.8 & 5.4 & & & \\
\hline External Debt Service to Exports (percent) & 2.9 & 2.6 & 3.3 & 2.8 & 4.4 & 6.3 & & & \\
\hline Public Domestic Debt (in percent of GDP) & 6.1 & 5.3 & 4.4 & 7.7 & 5.6 & 5.1 & & & \\
\hline Discount rate (percent) & 5.0 & 5.0 & 5.0 & 4.0 & 4.0 & 4.0 & 5.0 & 5.0 & 5.0 \\
\hline \multicolumn{10}{|c|}{ (In percent of GDP, unless indicated otherwise) } \\
\hline Real GDP (percentage change) & 5.3 & 5.2 & 6.0 & 4.1 & 5.4 & 5.9 & & & \\
\hline GDP Deflator (percentage change) & 12.1 & 6.2 & 5.3 & 11.0 & 6.7 & 6.1 & & & \\
\hline \multicolumn{10}{|l|}{ Fiscal } \\
\hline External Grants (incl. HIPC Relief) & 12.6[ & 9.8 & 8.6 & 11.7[ & 13.5 & 11.3 & & & \\
\hline Revenue (excl. External Grants) & 13.1 & 13.3 & 13.7 & 12.8 & 12.8 & 13.3 & & & \\
\hline Primary Expenditures & 26.6 & 25.8 & 25.2 & 25.1 & 26.9 & 25.7 & & & \\
\hline Primary Balance, incl. External Grants & -0.8 & -2.7 & -2.9 & -0.5 & -0.6 & -1.1 & & & \\
\hline Primary Balance, excl. External Grants & -13.4 & -12.5 & -11.4 & -12.2 & -14.1 & -12.4 & & & \\
\hline Grant Element of New External Borrowing (percent) ${ }^{2}$ & 53.3 & 50.5 & 57.6 & $\ldots$ & 12.3 & 6.8 & $\ldots$ & 18.9 & 13.4 \\
\hline \multicolumn{10}{|l|}{ Balance of Payments } \\
\hline Exports of Goods and Services & 9.0 & 10.4 & 11.2 & 10.2 & 10.9 & 11.4 & & & \\
\hline In millions of U.S. dollars & 450 & 549 & 637 & 534 & 621 & 701 & & & \\
\hline Imports of Goods and Services & 27.1 & 27.4 & 26.7 & 28.3 & 30.6 & 30.2 & & & \\
\hline In millions of U.S. dollars & 1,352 & 1,452 & 1,518 & 1,482 & 1,742 & 1,850 & & & \\
\hline Current Account, incl. Official Transfers & -6.6 & -9.5 & -9.0 & -7.3 & -7.9 & -8.8 & & & \\
\hline \multicolumn{10}{|c|}{$\begin{array}{l}\text { Source: Rwandan authorities, IMF, and World Bank. } \\
{ }^{1} \text { Conducted at the time of the sixth PRGF review; see IMF Country Report No 09/264. } \\
{ }^{2} \text { Includes publicly guaranteed external borrowing. }\end{array}$} \\
\hline
\end{tabular}

13. The main lesson from this scenario is that while the external debt dynamics appear sustainable under the baseline, there are risks - especially in the short term - associated with the additional nonconcessional external borrowing. It would therefore be imperative to continue to carefully assess the economic viability of large investment projects, and continue to consider financing options that crowd in participation from the private sector. In addition, it would also be important to carefully phase such projects so as to manage capacity constraints for implementation and avoid undermining macroeconomic stability (directly and indirectly from debt sustainability). The latter will become even more relevant when considering the phasing, implementation, and financing of the Bugesera Airport and regional railroad projects - easily the largest two of the six strategic investment projects.

\section{Public Sector DSA}

14. The results of the public sector DSA are similar to those of the external DSA. Given the assumption that there would be no net domestic borrowing beyond the near term, the public sector DSA indicators would gradually converge to those of the external DSA (see Appendix Tables 3-4 and Appendix Figure 2). Indeed, domestic debt would fall to 3.2 percent of GDP by 2015 and below 1 percent of GDP by 2030 . 


\section{DebT Distress Classification ANd Conclusions}

15. It is the staffs' view that Rwanda should be considered at moderate risk of debt distress based on external debt burden indicators. The public sector DSA results suggest that Rwanda's overall public sector debt dynamics are sustainable in light of the current size and evolution of the domestic debt stock.

The "moderate" rather than "low" rating of risk of debt distress is based on the vulnerabilities associated with the PV of debt-to-exports ratio. Careful vetting, prioritization, and sequencing of the implementation of investment projects by the Rwandan authorities, as well as strengthening implementation capacity, will be essential to maintain debt sustainability over the near and medium term. The authorities are well aware of these vulnerabilities and have identified several measures to help lift the export base. A comprehensive strategy for export development, expected to be submitted to Cabinet for approval in 2010, will outline policies to increase and diversify exports that would help reduce vulnerabilities to debt sustainability. In addition, deepening debt management capacity and building up a reliable framework for public-private partnerships would help the authorities identify and assess potentially highyielding projects and their risks, and further open up opportunities to crowd in the private sector. The authorities are also continuing to build capacity to conduct their own DSA's on a semi-annual basis. 
Appendix Table 1.: External Debt Sustainability Framework, Baseline Scenario, 2007-2030 1/

(In percent of GDP, unless otherwise indicated)

\begin{tabular}{|c|c|c|c|c|c|c|c|c|c|c|c|c|c|c|c|c|c|}
\hline & \multicolumn{3}{|c|}{ Actual } & \multirow{2}{*}{\multicolumn{2}{|c|}{$\begin{array}{c}\text { Historical } 0 \text { Standard } \\
\text { Average } 0 \text { Deviation }\end{array}$}} & \multicolumn{6}{|c|}{ Projections } & \multirow[b]{2}{*}{$\begin{array}{c}2010-2015 \\
\text { Average }\end{array}$} & \multirow[b]{2}{*}{2016} & \multirow[b]{2}{*}{2020} & \multirow[b]{2}{*}{2025} & \multirow[b]{2}{*}{2030} & \multirow[b]{2}{*}{$\begin{array}{c}2016-2030 \\
\text { Average }\end{array}$} \\
\hline & 2007 & 2008 & 2009 & & & 2010 & 2011 & 2012 & 2013 & 2014 & 2015 & & & & & & \\
\hline External debt (nominal) 1/ & 15.2 & 14.8 & 14.4 & & & 16.4 & 19.3 & 19.1 & 18.0 & 16.7 & 15.4 & & 14.8 & 16.4 & 20.7 & 22.7 & \\
\hline $\mathrm{o} / \mathrm{w}$ public and publicly guaranteed $(\mathrm{PPG})$ & 15.2 & 14.8 & 14.4 & & & 16.4 & 193 & 191 & 18.0 & 167 & 154 & & 14.8 & 164 & 207 & 227 & \\
\hline Change in external debt & -0.3 & -0.5 & -0.3 & & & 2.0 & 2.9 & -0.2 & -1.1 & -1.3 & -1.3 & & -0.7 & 1.0 & 0.8 & 0.2 & \\
\hline Identified net debt-creating flows & -2.6 & -0.4 & 3.5 & & & 5.4 & 6.5 & 2.7 & 1.5 & 0.9 & 0.6 & & 1.9 & 0.9 & -1.2 & -3.3 & \\
\hline Non-interest current account deficit & 2.1 & 4.8 & 7.1 & 2.1 & 2.9 & 7.7 & 8.5 & 5.0 & 4.0 & 3.4 & 3.2 & & 4.6 & 4.1 & 2.2 & 0.2 & 2.8 \\
\hline Deficit in balance of goods and services & 14.1 & 15.2 & 18.1 & & & 19.7 & 18.8 & 14.9 & 13.0 & 11.8 & 11.0 & & 10.7 & 9.3 & 7.5 & 5.8 & \\
\hline Exports & 11.1 & 14.6 & 10.2 & & & 10.9 & 11.4 & 11.8 & 12.3 & 12.8 & 13.4 & & 13.7 & 14.9 & 16.4 & 17.9 & \\
\hline Imports & 25.2 & 29.9 & 28.3 & & & 30.6 & 30.2 & 26.7 & 25.3 & 24.6 & 24.4 & & 24.3 & 24.1 & 23.9 & 23.6 & \\
\hline Net current transfers (negative $=$ in flow) & -12.3 & -11.0 & -11.5 & -12.3 & 1.4 & -12.8 & -11.2 & -10.8 & -9.8 & -9.2 & -8.6 & & -6.7 & -5.9 & -6.0 & -6.3 & -6.2 \\
\hline & -9.7 & -9.5 & -10.0 & & & -11.4 & -9.8 & -9.4 & -8.5 & -7.8 & -7.1 & & -5.2 & -4.5 & -4.6 & -4.9 & \\
\hline Other current account flows (negative $=$ net inflow) & 0.3 & 0.6 & 0.6 & & & 0.8 & 1.0 & 0.9 & 0.8 & 0.8 & 0.7 & & 0.6 & 0.7 & 0.7 & 0.7 & \\
\hline Net FDI (negative = inflow) & -2.2 & -2.2 & -2.3 & -1.0 & 0.9 & -1.8 & -1.4 & -1.6 & -1.8 & -1.8 & -1.9 & & -2.0 & -2.4 & -2.4 & -2.4 & -2.3 \\
\hline Endogenous debt dynamics $2 /$ & -2.5 & -2.9 & -1.4 & & & -0.5 & -0.7 & -0.7 & -0.8 & -0.7 & -0.7 & & -0.7 & -0.7 & -1.0 & -1.1 & \\
\hline $\begin{array}{l}\text { Contribution from nominal interest rate } \\
\text { Contribution from real GDP growth }\end{array}$ & 0.2 & 0.1 & 0.1 & & & 0.3 & 0.2 & 0.4 & 0.4 & 0.3 & 0.3 & & 0.3 & 0.2 & 0.2 & 0.2 & \\
\hline $\begin{array}{l}\text { Contribution from real GDP growth } \\
\text { Contribution from rpice and exchange rate changes }\end{array}$ & -0.7 & -1.4 & $\begin{array}{c}-0.5 \\
-1.0\end{array}$ & & & -0.7 & -0.9 & -1.1 & -1.2 & -1.1 & -1.0 & & -0.9 & -0.9 & -1.2 & -1.3 & \\
\hline Residual (3-4) 3/ & $\begin{array}{l}-1.9 \\
2.3\end{array}$ & $\begin{array}{l}-0.1 \\
-0.1\end{array}$ & -1.8 & & & -3.4 & -3.7 & -2.9 & -2.6 & -2.1 & -1.8 & & -2.6 & 0.1 & 1.9 & 3.5 & \\
\hline $\mathrm{o} / \mathrm{w}$ exceptional financing & 0.0 & 0.0 & 0.0 & & & 0.0 & 0.0 & 0.0 & 0.0 & 0.0 & 0.0 & & 0.0 & 0.0 & 0.0 & 0.0 & \\
\hline PV of external debt $4 /$ & $\ldots$ & $\ldots$ & 9.1 & & & 11.2 & 14.3 & 14.3 & 13.2 & 12.1 & 11.0 & & 10.3 & 10.6 & 13.3 & 14.8 & \\
\hline In percent of exports & $\ldots$ & $\ldots$ & 89.0 & & & 102.8 & 125.2 & 120.6 & 107.4 & 94.2 & 82.2 & & 75.5 & 71.4 & 81.5 & 83.0 & \\
\hline PV of PPG external debt & ... & $\ldots$ & 9.1 & & & 11.2 & 14.3 & 14.3 & 13.2 & 12.1 & 11.0 & & 10.3 & 10.6 & 13.3 & 14.8 & \\
\hline In percent of exports & ... & ... & 89.0 & & & 102.8 & 125.2 & 120.6 & 107.4 & 94.2 & 82.2 & & 75.5 & 71.4 & 81.5 & 83.0 & \\
\hline In percent of government revenues & $\ldots$ & $\ldots$ & 70.6 & & & 87.7 & 108.0 & 103.8 & 92.6 & 82.4 & 74.0 & & 68.9 & 68.6 & 82.9 & $\mathbf{8 8 . 7}$ & \\
\hline Debt service-to-exports ratio (in percent) & 3.5 & 2.1 & 2.8 & & & 4.4 & 6.3 & 6.9 & 8.6 & 8.2 & 7.5 & & 6.6 & 4.7 & 3.8 & 4.1 & \\
\hline PPG debt service-to-exports ratio (in percent) & 3.5 & 2.1 & 2.8 & & & 4.4 & 6.3 & 6.9 & 8.6 & 8.2 & 7.5 & & 6.6 & 4.7 & 3.8 & 4.1 & \\
\hline PPG debt service-to-revenue ratio (in percent) & 3.1 & 2.1 & 2.2 & & & 3.8 & 5.4 & 5.9 & 7.4 & 7.1 & 6.7 & & 6.0 & 4.5 & 3.9 & 4.4 & \\
\hline $\begin{array}{l}\text { Total gross financing need (Billions of U.S. dollars) } \\
\text { Non-interest current account deficit that tabilizes dat ratio }\end{array}$ & $\begin{array}{l}0.0 \\
2.4\end{array}$ & $\begin{array}{l}0.1 \\
5.2\end{array}$ & $\begin{array}{l}0.3 \\
7.5\end{array}$ & & & $\begin{array}{l}0.4 \\
5.7\end{array}$ & $\begin{array}{l}0.5 \\
5.7\end{array}$ & $\begin{array}{l}0.3 \\
5.2\end{array}$ & $\begin{array}{l}0.2 \\
5.2\end{array}$ & $\begin{array}{l}0.2 \\
4.7\end{array}$ & $\begin{array}{l}0.2 \\
4.5\end{array}$ & & $\begin{array}{l}0.3 \\
5.3\end{array}$ & $\begin{array}{l}0.3 \\
3.1\end{array}$ & $\begin{array}{l}0.1 \\
1.5\end{array}$ & -0.4 & \\
\hline \multicolumn{18}{|l|}{ Key macroeconomic assumptions } \\
\hline Real GDP growth (in percent) & 5.5 & 11.2 & 4.1 & 7.9 & 3.3 & 5.4 & 5.9 & 6.4 & 6.9 & 6.5 & 6.5 & 6.3 & 6.5 & 6.5 & 6.5 & 6.5 & 6.5 \\
\hline GDP deflator in US dollar terms (change in percent) & 14.2 & 12.7 & 7.3 & 3.7 & 10.6 & 3.0 & 1.6 & 2.1 & 1.9 & 1.9 & 1.9 & 2.1 & 2.0 & 2.0 & 2.0 & 2.0 & 2.0 \\
\hline Effective interest rate (percent) $5 /$ & 1.3 & 1.2 & 1.1 & 1.1 & 0.2 & 1.9 & 1.6 & 2.2 & 2.3 & 2.1 & 1.9 & 2.0 & 1.8 & 1.2 & 1.1 & 1.2 & 1.3 \\
\hline Growth of exports of G\&S (US dollar terms, in percent) & 19.6 & 64.7 & -22.4 & 16.5 & 24.6 & 16.4 & 12.9 & 12.2 & 13.0 & 13.3 & 13.3 & 13.5 & 11.1 & 10.9 & 10.7 & 10.5 & 10.8 \\
\hline Growth of imports of G\&S (US dollar terms, in percent) & 21.1 & 48.3 & 5.8 & 13.9 & 16.5 & 17.5 & 6.2 & -4.0 & 3.0 & 5.9 & 7.5 & 6.0 & 8.4 & 8.4 & 8.4 & 8.4 & 8.4 \\
\hline Grant element of new public sector borrowing (in percent) & & & & $\ldots$ & ... & 12.3 & 6.8 & 23.5 & 43.0 & 42.7 & 42.3 & 28.4 & 42.0 & 40.5 & 39.2 & 37.9 & 39.8 \\
\hline Government revenues (excluding grants, in percent of GDP) & 12.3 & 14.9 & 12.8 & & & 12.8 & 13.3 & 13.8 & 14.2 & 14.6 & 14.8 & & 15.0 & 15.5 & 16.1 & 16.7 & 15.8 \\
\hline Aid flows (in Billions of US dollars) $7 /$ & 0.4 & 0.6 & 0.7 & & & 0.8 & 0.7 & 0.7 & 0.7 & 0.7 & 0.7 & & 0.8 & 0.9 & 1.3 & 1.8 & \\
\hline $\mathrm{o} / \mathrm{w}$ Grants & 0.3 & 0.5 & 0.6 & & & 0.8 & 0.7 & 0.7 & 0.7 & 0.7 & 0.7 & & 0.7 & 0.7 & 0.9 & 1.3 & \\
\hline $\mathrm{o} / \mathrm{w}$ Concessional loans & 0.1 & 0.1 & 0.1 & & & 0.0 & 0.1 & 0.0 & 0.1 & 0.0 & 0.0 & & 0.1 & 0.2 & 0.3 & 0.4 & \\
\hline Grant-equivalent financing (in percent of GDP) $8 /$ & $\ldots$ & $\ldots$ & & & & 13.8 & 11.5 & 11.1 & 9.9 & 9.0 & 8.1 & & 7.7 & 6.3 & 5.8 & 5.4 & 6.2 \\
\hline Grant-equivalent financing (in percent of external financing) 8/ & $\ldots$ & $\ldots$ & $\ldots$ & & & 83.6 & 73.3 & 88.6 & 94.1 & 94.9 & 94.9 & & 91.7 & 79.8 & 77.8 & 78.1 & 80.9 \\
\hline \multicolumn{18}{|l|}{ Memorand } \\
\hline Nominal GDP (Billions of US dollars) & 3.7 & 4.7 & 5.2 & & & 5.7 & 6.1 & 6.6 & 7.2 & 7.9 & 8.5 & & 9.3 & 12.9 & 19.6 & 29.6 & \\
\hline Nominal dollar GDP growth & 20.5 & 25.3 & 11.8 & & & 8.5 & 7.6 & 8.6 & 9.0 & 8.6 & 8.6 & 8.5 & 8.6 & 8.6 & 8.6 & 8.6 & 8.6 \\
\hline PV of PPG external debt (in Billions of US dollars) & & & 0.5 & & & 0.6 & 0.9 & 0.9 & 0.9 & 0.9 & 0.9 & & 0.9 & 1.4 & 2.6 & 4.3 & \\
\hline (PVt-PVt-1)/GDPt-1 (in percent) & & & & & & 2.9 & 4.2 & 1.2 & 0.1 & -0.1 & -0.1 & 1.4 & 0.2 & 1.4 & 1.6 & 1.4 & 1.3 \\
\hline $\begin{array}{l}\text { Gross remittances (Billions of US dollars) } \\
\text {. }\end{array}$ & 0.1 & 0.1 & 0.1 & & & 0.1 & 0.1 & 0.1 & 0.1 & 0.2 & 0.2 & & 0.2 & 0.3 & 0.4 & 0.6 & \\
\hline PV of PPG external debt (in percent of GDP + remittances) & ... & ... & 8.9 & & & 11.0 & 14.0 & 14.0 & 12.9 & 11.8 & 10.8 & & 10.1 & 10.4 & 13.1 & 14.5 & \\
\hline $\begin{array}{l}\text { PV of PPG external debt (in percent of exports + remittances) } \\
\text { Debt service of PPG external debt (in percent of exports + remittances) }\end{array}$ & $\cdots$ & $\cdots$ & 72.4 .2 & & & $\begin{array}{ll}85.2 \\
37\end{array}$ & $\begin{array}{r}105.2 \\
5.3\end{array}$ & 102.4 & 92.2 & $\begin{array}{l}81.2 \\
70\end{array}$ & $\begin{array}{l}71.1 \\
65\end{array}$ & & 65.5 & 62.6 & $\begin{array}{r}72.3 \\
3\end{array}$ & $\begin{array}{r}74.3 \\
\end{array}$ & \\
\hline Debt service of PPG external debt (in percent of exports + remittances) & $\ldots$ & $\ldots$ & 2.2 & & & 3.7 & 5.3 & 5.8 & & & 6.5 & & & & & & \\
\hline
\end{tabular}

$1 /$ Includes both public and private sector external debt.

2/ Derived as $[\mathrm{r}-\mathrm{g}-\rho(1+\mathrm{g})](1+\mathrm{g}+\rho+\mathrm{g} \rho)$ times previous period debt ratio, with $\mathrm{r}=$ nominal interest rate; $\mathrm{g}=$ real GDP growth rate, and $\rho=$ growth rate of GDP deflator in U.S. dollar terms.

5/ Assumes that PV of private sector debt is equivalent to its face value.

6/ Historical averages and standard deviations are generally derived over the past 10 years, subject to data availability.

G 
Appendix Table 2.Rwanda: Sensitivity Analysis for Key Indicators of Public and Publicly Guaranteed External Debt, 2010-2030

(In percent)

\begin{tabular}{|c|c|c|c|c|c|c|c|c|}
\hline & \multicolumn{8}{|c|}{ Projections } \\
\hline & 2010 & 2011 & 2012 & 2013 & 2014 & 2015 & 2020 & 2030 \\
\hline \multicolumn{9}{|c|}{ PV of de bt-to GDP ratio } \\
\hline Baseline & 11 & 14 & 14 & 13 & 12 & 11 & 11 & 15 \\
\hline \multicolumn{9}{|l|}{ A. Alternative Scenarios } \\
\hline A1. Key variables at their historical averages in $2010-20301 /$ & 11 & 9 & 8 & 6 & 5 & 4 & 2 & 19 \\
\hline A2. New public sector loans on less favorable terms in $2010-20302$ & 11 & 15 & 16 & 15 & 14 & 13 & 15 & 24 \\
\hline \multicolumn{9}{|l|}{ B. Bound Tests } \\
\hline B1. Real GDP growth at historical average minus one standard deviation in 2011-2012 & 11 & 14 & 14 & 13 & 12 & 11 & 11 & 15 \\
\hline B2. Export value growth at historical average minus one standard deviation in 2011-2012 3/ & 11 & 16 & 18 & 17 & 16 & 14 & 13 & 15 \\
\hline B3. US dollar GDP deflator at historical average minus one standard deviation in 2011-2012 & 11 & 15 & 17 & 16 & 14 & 13 & 12 & 17 \\
\hline B4. Net non-debt creating flows at historical average minus one standard deviation in 2011-2012 4/ & 11 & 15 & 16 & 15 & 14 & 13 & 12 & 15 \\
\hline B5. Combination of B1-B4 using one-half standard deviation shocks & 11 & 16 & 18 & 17 & 16 & 14 & 13 & 16 \\
\hline B6. One-time 30 percent nominal depreciation relative to the baseline in 20115 / & 11 & 20 & 20 & 18 & 17 & 15 & 15 & 21 \\
\hline
\end{tabular}

PV of debt-to-exports ratio

Baseline

A. Alternative Scenarios

A1. Key variables at their historical averages in 2010-2030 1/

A2. New public sector loans on less favorable terms in 2010-2030 2

B. Bound Tests

B1. Real GDP growth at historical average minus one standard deviation in 2011-2012

B2. Export value growth at historical average minus one standard deviation in 2011-20123/

B3. US dollar GDP deflator at historical average minus one standard deviation in 2011-2012

B4. Net non-debt creating flows at historical average minus one standard deviation in 2011-2012 4/

B5. Combination of B1-B4 using one-half standard deviation shocks

B6. One-time 30 percent nominal depreciation relative to the baseline in 2011 5/ $\begin{array}{llllllll}103 & 125 & 121 & 107 & 94 & 82 & \mathbf{7 1} & 83\end{array}$

$\begin{array}{llllllll}103 & 82 & 65 & 49 & 37 & 29 & \mathbf{1 6} & 107\end{array}$

$\begin{array}{lrrrrrrr}103 & 132 & 134 & 124 & 112 & 101 & \mathbf{1 0 3} & 133\end{array}$

$\begin{array}{rrrrrrrr}103 & 123 & 119 & 106 & 93 & 81 & \mathbf{7 0} & 82 \\ 103 & 167 & 231 & 207 & 183 & 162 & \mathbf{1 3 1} & 130 \\ 103 & 123 & 119 & 106 & 93 & 81 & \mathbf{7 0} & 82 \\ 103 & 133 & 136 & 122 & 107 & 94 & \mathbf{7 9} & 84 \\ 103 & 144 & 169 & 151 & 133 & 117 & \mathbf{9 6} & 99 \\ 103 & 123 & 119 & 106 & 93 & 81 & \mathbf{7 0} & 82\end{array}$

\section{PV of debt-to-revenue ratio}

Baseline

$\begin{array}{rrrrrrrr}88 & 108 & 104 & 93 & 82 & 74 & \mathbf{6 9} & 89 \\ & & & & & & & \\ 88 & 71 & 56 & 42 & 33 & 26 & \mathbf{1 6} & 114 \\ 88 & 114 & 116 & 107 & 98 & 91 & \mathbf{9 9} & 143 \\ & & & & & & & \\ & & & & & & & \\ 88 & 107 & 105 & 94 & 83 & 75 & \mathbf{6 9} & 90 \\ 88 & 117 & 132 & 119 & 107 & 97 & \mathbf{8 4} & 92 \\ 88 & 116 & 122 & 109 & 97 & 87 & \mathbf{8 1} & 104 \\ 88 & 115 & 117 & 105 & 94 & 85 & \mathbf{7 5} & 90 \\ 88 & 118 & 133 & 119 & 107 & 97 & \mathbf{8 5} & 97 \\ 88 & 150 & 145 & 129 & 115 & 103 & \mathbf{9 6} & 124\end{array}$

A. Alternative Scenarios

A1. Key variables at their historical averages in 2010-2030 1/

A2. New public sector loans on less favorable terms in 2010-2030 2

B. Bound Tests

B1. Real GDP growth at historical average minus one standard deviation in 2011-2012 B2. Export value growth at historical average minus one standard deviation in 2011-2012 3 /

B3. US dollar GDP deflator at historical average minus one standard deviation in 2011-2012 B4. Net non-debt creating flows at historical average minus one standard deviation in 2011-2012 4/

B5. Combination of B1-B4 using one-half standard deviation shocks

B6. One-time 30 percent nominal depreciation relative to the baseline in 20115 / 
Appendix Table 2.Rwanda: Sensitivity Analysis for Key Indicators of Public and Publicly Guaranteed External Debt, $2010-2030$ (continued)

(In percent)

Debt service-to-exports ratio

Baseline

A. Alternative Scenarios

A1. Key variables at their historical averages in 2010-2030 1/

A2. New public sector loans on less favorable terms in 2010-2030 2

\section{B. Bound Tests}

B1. Real GDP growth at historical average minus one standard deviation in 2011-2012

B2. Export value growth at historical average minus one standard deviation in 2011-2012 3/

B3. US dollar GDP deflator at historical average minus one standard deviation in 2011-2012

B4. Net non-debt creating flows at historical average minus one standard deviation in 2011-2012 4/

B5. Combination of B1-B4 using one-half standard deviation shocks

B6. One-time 30 percent nominal depreciation relative to the baseline in 20115 /

$\begin{array}{llllllll}4 & 6 & 7 & 9 & 8 & 7 & \mathbf{5} & 4 \\ & & & & & & & \\ 4 & 6 & 6 & 7 & 6 & 6 & \mathbf{2} & 3 \\ 4 & 6 & 6 & 6 & 6 & 8 & \mathbf{5} & 6\end{array}$

\section{Debt service-to-revenue ratio}

Baseline

\section{A. Alternative Scenarios}

A1. Key variables at their historical averages in 2010-2030 1/

\section{B. Bound Tests}

B1. Real GDP growth at historical average minus one standard deviation in 2011-2012 B2. Export value growth at historical average minus one standard deviation in 2011-2012 3/

B3. US dollar GDP deflator at historical average minus one standard deviation in 2011-2012

B4. Net non-debt creating flows at historical average minus one standard deviation in 2011-2012 4/

B5. Combination of B1-B4 using one-half standard deviation shocks

B6. One-time 30 percent nominal depreciation relative to the baseline in 20115

Memorandum item:

Grant element assumed on residual financing (i.e., financing required above baseline) 6 / A2. New public sector loans on less favorable terms in 2010-20302

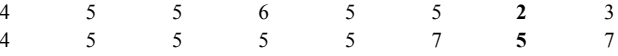

Sources: Country authorities; and staff estimates and projections.

1/ Variables include real GDP growth, growth of GDP deflator (in U.S. dollar terms), non-interest current account in percent of GDP, and non-debt creating flows

2/ Assumes that the interest rate on new borrowing is by 2 percentage points higher than in the baseline., while grace and maturity periods are the same as in the baseline.

3/ Exports values are assumed to remain permanently at the lower level, but the current account as a share of GDP is assumed to return to its baseline level after the shock (implicitly assuming an offsetting adjustment in import levels).

4/ Includes official and private transfers and FDI.

5/ Depreciation is defined as percentage decline in dollar/local currency rate, such that it never exceeds 100 percent.

6/ Applies to all stress scenarios except for A2 (less favorable financing) in which the terms on all new financing are as specified in footnote 2. 
Appendix Figure 1. Rwanda: Indicators of Public and Publicly Guaranteed External Debt under Alternative Scenarios, 2010-2030 1/
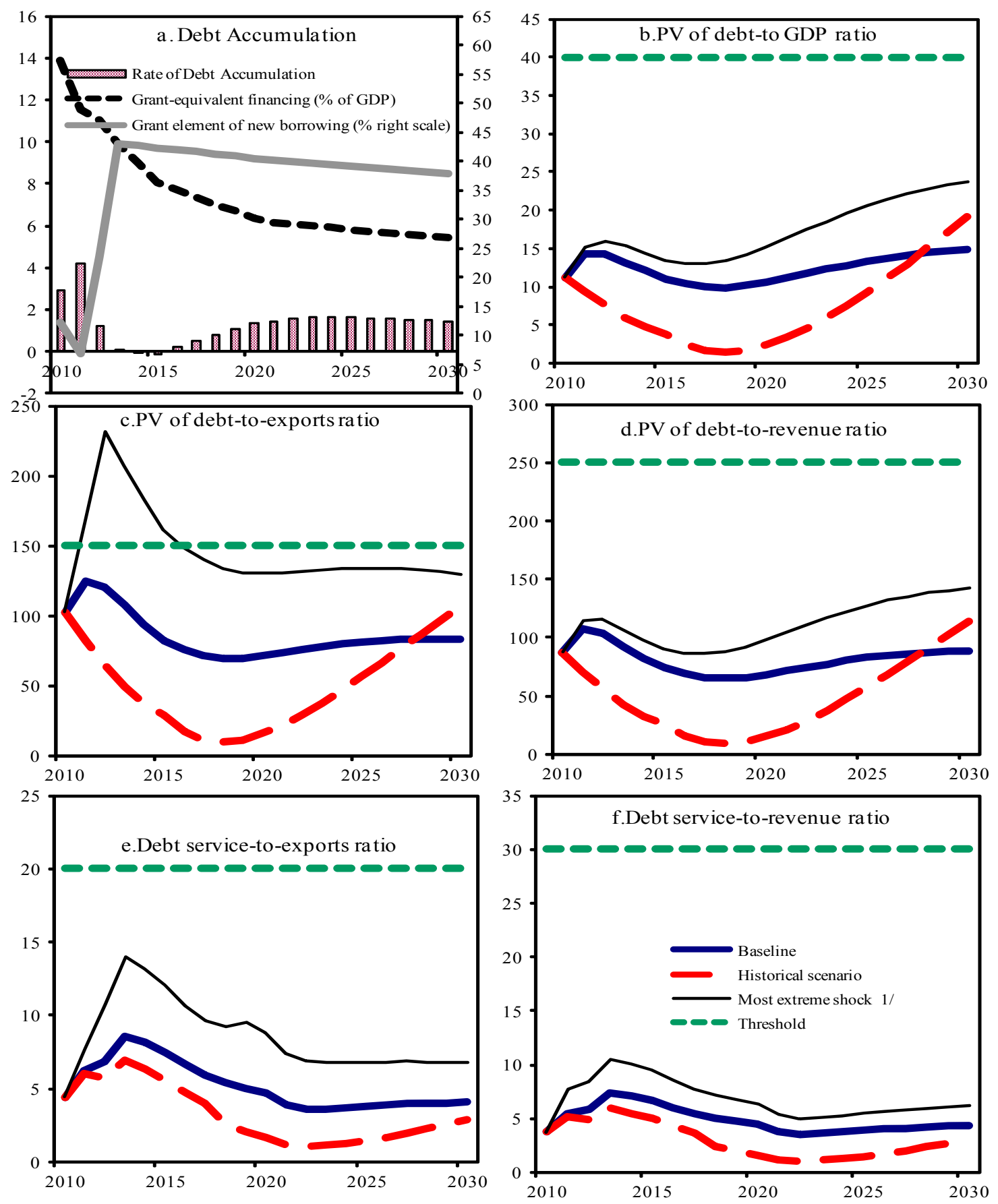

Sources: Country authorities; and staff estimates and projections.

$1 /$ The most extreme stress test is the test that yields the highest ratio in 2020. In figure b. it corresponds to a Terms shock; in c. to a Exports shock; in d. to a Terms shock; in e. to a Exports shock and in figure f. to a One-time depreciation shock 
Appendix Table 3.Rwanda: Public Sector Debt Sustainability Framework, Baseline Scenario, 2007-2030 (In percent of GDP, unless otherwise indicated)

\begin{tabular}{|c|c|c|c|c|c|c|c|c|c|c|c|c|c|c|c|}
\hline & \multicolumn{3}{|c|}{ Actual } & \multirow[b]{2}{*}{ Average } & \multirow[b]{2}{*}{$\begin{array}{r}\text { Standard } \\
\text { Deviation }\end{array}$} & \multicolumn{5}{|l|}{ Estimate } & \multicolumn{5}{|c|}{ Projections } \\
\hline & 2007 & 2008 & 2009 & & & 2010 & 2011 & 2012 & 2013 & 2014 & 2015 & $\begin{array}{l}2010-15 \\
\text { Average }\end{array}$ & 2020 & 2030 & $\begin{array}{l}2016-30 \\
\text { Average }\end{array}$ \\
\hline $\begin{array}{l}\text { Public sector debt } 1 / \\
\text { o/w foreign-currency denominated }\end{array}$ & $\begin{array}{l}27.1 \\
15.2\end{array}$ & $\begin{array}{l}21.6 \\
14.8\end{array}$ & $\begin{array}{l}22.1 \\
14.4\end{array}$ & & & $\begin{array}{l}22.1 \\
16.4\end{array}$ & $\begin{array}{l}24.3 \\
19.3\end{array}$ & $\begin{array}{l}23.6 \\
19.1\end{array}$ & $\begin{array}{l}22.0 \\
18.0\end{array}$ & $\begin{array}{l}20.3 \\
16.7\end{array}$ & $\begin{array}{l}18.6 \\
15.4\end{array}$ & & $\begin{array}{l}18.2 \\
16.4\end{array}$ & $\begin{array}{l}23.3 \\
22.7\end{array}$ & \\
\hline Change in public sector debt & 0.5 & -5.5 & 0.6 & & & -0.1 & 2.3 & -0.7 & -1.6 & -1.7 & -1.7 & & 0.8 & 0.1 & \\
\hline Identified debt-creating flows & -2.7 & -5.2 & -1.4 & & & -0.7 & -0.1 & -1.0 & -1.4 & -1.3 & -1.3 & & 0.9 & 0.1 & \\
\hline Primary deficit & 0.9 & -0.8 & 0.6 & 0.7 & 1.6 & 0.5 & 1.1 & 0.4 & 0.0 & -0.1 & -0.1 & 0.3 & 2.1 & 1.7 & 1.8 \\
\hline Revenue and grants & 21.2 & 25.6 & 24.4 & & & 26.3 & 24.5 & 24.4 & 23.7 & 23.3 & 22.6 & & 20.7 & 21.2 & \\
\hline of which: grants & 8.8 & 10.8 & 11.6 & & & 13.5 & 11.2 & 10.6 & 9.5 & 8.6 & 7.8 & & 5.2 & 4.5 & \\
\hline Primary (noninterest) expenditure & 22.1 & 24.8 & 25.1 & & & 26.8 & 25.6 & 24.8 & 23.7 & 23.2 & 22.5 & & 22.8 & 22.9 & \\
\hline Automatic debt dynamics & -3.8 & -4.6 & -2.2 & & & -1.3 & -1.4 & -1.6 & -1.5 & -1.3 & -1.3 & & -1.2 & -1.6 & \\
\hline Contribution from interest rate/growth differential & -2.4 & -3.7 & -1.3 & & & -1.2 & -1.3 & -1.5 & -1.5 & -1.3 & -1.3 & & -1.2 & -1.6 & \\
\hline of which: contribution from average real interest rate & -1.0 & -1.0 & -0.4 & & & -0.1 & -0.1 & 0.0 & 0.1 & 0.0 & 0.0 & & -0.1 & -0.2 & \\
\hline of which: contribution from real GDP growth & -1.4 & -2.7 & -0.9 & & & -1.1 & -1.2 & -1.5 & -1.5 & -1.3 & -1.2 & & -1.1 & -1.4 & \\
\hline Contribution from real exchange rate depreciation & -1.4 & -0.9 & -1.0 & & & -0.1 & -0.1 & -0.1 & 0.0 & 0.0 & 0.0 & & $\ldots$ & & \\
\hline Other identified debt-creating flows & 0.3 & 0.2 & 0.3 & & & 0.1 & 0.1 & 0.1 & 0.1 & 0.1 & 0.1 & & 0.0 & 0.0 & \\
\hline Privatization receipts (negative) & 0.0 & 0.0 & 0.0 & & & 0.0 & 0.0 & 0.0 & 0.0 & 0.0 & 0.0 & & 0.0 & 0.0 & \\
\hline Recognition of implicit or contingent liabilities & 0.0 & 0.0 & 0.0 & & & 0.0 & 0.0 & 0.0 & 0.0 & 0.0 & 0.0 & & 0.0 & 0.0 & \\
\hline Debt relief (HIPC and other) & -0.1 & -0.1 & -0.1 & & & -0.1 & -0.1 & -0.1 & -0.1 & -0.1 & -0.1 & & 0.0 & 0.0 & \\
\hline Other (specify, e.g. bank recapitalization) & 0.4 & 0.3 & 0.4 & & & 0.2 & 0.2 & 0.2 & 0.2 & 0.2 & 0.1 & & 0.0 & 0.0 & \\
\hline Residual, including asset changes & 3.2 & -0.3 & 1.9 & & & 0.6 & 2.4 & 0.3 & -0.2 & -0.3 & -0.3 & & -0.2 & 0.0 & \\
\hline \multicolumn{16}{|l|}{ Other $S$ ustainability Indicators } \\
\hline PV of public sector debt & 11.8 & 6.8 & 16.8 & & & 16.9 & 19.4 & 18.8 & 17.2 & 15.6 & 14.2 & & 12.4 & 15.4 & \\
\hline $\mathrm{o} / \mathrm{w}$ foreign-currency denominated & 0.0 & 0.0 & 9.1 & & & 11.2 & 14.3 & 14.3 & 13.2 & 12.1 & 11.0 & & 10.6 & 14.8 & \\
\hline $\mathrm{o} / \mathrm{w}$ external & $\ldots$ & $\ldots$ & 9.1 & & & 11.2 & 14.3 & 14.3 & 13.2 & 12.1 & 11.0 & & 10.6 & 14.8 & \\
\hline PV of contingent liabilities (not included in public sector debt) & $\ldots$ & $\ldots$ & $\ldots$ & & & $\ldots$ & $\ldots$ & $\ldots$ & & $\ldots$ & & & $\ldots$ & & \\
\hline Gross financing need $2 /$ & 1.7 & -0.1 & 1.1 & & & 4.0 & 3.8 & 3.2 & 3.0 & 2.9 & 2.7 & & 4.3 & 2.9 & \\
\hline $\mathrm{PV}$ of public sector debt-to-revenue and grants ratio (in percent) & 55.8 & 26.5 & 68.6 & & & 64.2 & 79.2 & 77.0 & 72.5 & 67.3 & 62.8 & & 60.1 & 72.7 & \\
\hline $\mathrm{PV}$ of public sector debt-to-revenue ratio (in percent) & 95.8 & 45.8 & 130.6 & & & 131.7 & 146.1 & 136.5 & 120.7 & 106.9 & 95.6 & & 80.5 & 92.3 & \\
\hline o/w external $3 /$ & & & 70.6 & & & 87.7 & 108.0 & 103.8 & 92.6 & 82.4 & 74.0 & & 68.6 & 88.7 & \\
\hline Debt service-to-revenue and grants ratio (in percent) $4 /$ & 3.8 & 2.5 & 2.2 & & & 2.8 & 3.9 & 4.2 & 5.3 & 5.1 & 5.0 & & 3.8 & 3.6 & \\
\hline Debt service-to-revenue ratio (in percent) $4 /$ & 6.6 & 4.4 & 4.1 & & & 5.7 & 7.2 & 7.4 & 8.9 & 8.2 & 7.7 & & 5.0 & 4.5 & \\
\hline Primary deficit that stabilizes the debt-to-GDP ratio & 0.4 & 4.7 & 0.0 & & & 0.6 & -1.2 & 1.1 & 1.6 & 1.6 & 1.5 & & 1.4 & 1.6 & \\
\hline \multicolumn{16}{|l|}{ Key macroeconomic and fiscal assumptions } \\
\hline Real GDP growth (in percent) & 5.5 & 11.2 & 4.1 & 7.9 & 3.3 & 5.4 & 5.9 & 6.4 & 6.9 & 6.5 & 6.5 & 6.3 & 6.5 & 6.5 & 6.5 \\
\hline Average nominal interest rate on forex debt (in percent) & 1.3 & 1.2 & 1.1 & 1.1 & 0.2 & 1.9 & 1.6 & 2.2 & 2.3 & 2.1 & 1.9 & 2.0 & 1.2 & $1.2^{\prime}$ & 1.3 \\
\hline Average real interest rate on domestic debt (in percent) & -7.6 & -7.9 & -6.1 & -6.4 & 1.8 & -2.9 & -1.2 & -1.1 & 0.1 & -0.7 & -0.5 & -1.1 & -0.5 & $-0.3^{\prime \prime}$ & -0.4 \\
\hline Real exchange rate depreciation (in percent, + indicates depreciation) & -9.8 & -6.8 & -6.8 & -0.4 & 12.3 & -0.8 & $\ldots$ & $\ldots$ & & $\ldots$ & $\ldots$ & $\ldots$ & $\ldots$ & & \\
\hline Inflation rate (GDP deflator, in percent) & 13.2 & 12.6 & 11.0 & 8.9 & 7.5 & 6.7 & 6.1 & 5.6 & 5.0 & 5.0 & 5.0 & 5.6 & 5.0 & 5.0 & 5.0 \\
\hline Growth of real primary spending (deflated by GDP deflator, in percent) & 0.1 & 0.3 & 0.1 & 0.1 & 0.1 & 0.1 & 0.0 & 0.0 & 0.0 & 0.0 & 0.0 & 0.0 & 0.1 & 0.1 & 0.1 \\
\hline Grant element of new external borrowing (in percent) & $\ldots$ & $\ldots$ & $\ldots$ & $\ldots$ & $\ldots$ & 12.3 & 6.8 & 23.5 & 43.0 & 42.7 & 42.3 & 28.4 & 40.5 & 37.9 & $\ldots$ \\
\hline
\end{tabular}

Sources: Country authorities; and staff estimates and projections.

[nicate coverage of public sector, e.g., general government or nonfinancial public sector. Also whether net or gross debt is used.]

$2 /$ Gross financing need is defined as the primary deficit plus debt service plus the stock of short-term debt at the end of the last period

/ Revenues excluding grants.

4/ Debt service is defined as the sum of interest and amortization of medium and long-term debt.

$5 /$ Historical averages and standard deviations are generally derived over the past 10 years, subject to data availability. 
Appendix Table 4.Rwanda: Sensitivity Analysis for Key Indicators of Public Debt 2010-2030

Projections

\begin{tabular}{llllllll}
2010 & 2011 & 2012 & 2013 & 2014 & 2015 & 2020 & 2030 \\
\hline
\end{tabular}

PV of Debt-to-GDP Ratio

Bas eline

\section{A. Alternative scenarios}

A1. Real GDP growth and primary balance are at historical averages

A2. Primary balance is unchanged from 2010

A3. Permanently lower GDP growth 1/

\section{B. Bound tests}

B1. Real GDP growth is at historical average minus one standard deviations in 2011-2012

B2. Primary balance is at historical average minus one standard deviations in 2011-2012

B3. Combination of B1-B2 using one half standard deviation shocks

B4. One-time 30 percent real depreciation in 2011

B5. 10 percent of GDP increase in other debt-creating flows in 2011

PV of Debt-to-Revenue Ratio 2/

Bas eline

\section{A. Alternative scenarios}

A1. Real GDP growth and primary balance are at historical averages

A2. Primary balance is unchanged from 2010

A3. Permanently lower GDP growth 1/

\section{B. Bound tests}

B1. Real GDP growth is at historical average minus one standard deviations in 2011-2012

B2. Primary balance is at historical average minus one standard deviations in 2011-2012

B3. Combination of B1-B2 using one half standard deviation shocks

B4. One-time 30 percent real depreciation in 2011

B5. 10 percent of GDP increase in other debt-creating flows in 2011

\section{Debt Service-to-Revenue Ratio $2 /$}

Baseline

$\begin{array}{llllllll}17 & 20 & 20 & 19 & 17 & 16 & 16 & 22 \\ 17 & 21 & 22 & 20 & 18 & 17 & 15 & 17 \\ 17 & 20 & 20 & 18 & 17 & 15 & 13 & 15 \\ 17 & 24 & 23 & 21 & 20 & 18 & 15 & 17 \\ 17 & 29 & 28 & 26 & 24 & 23 & 19 & 20\end{array}$

\section{A. Alternative scenarios}

A1. Real GDP growth and primary balance are at historical averages A2. Primary balance is unchanged from 2010

A3. Permanently lower GDP growth 1/

$\begin{array}{llllllll}17 & 19 & 19 & 17 & 16 & 14 & 12 & 15\end{array}$

$\begin{array}{rrrrrrrr}17 & 19 & 18 & 17 & 16 & 15 & 10 & 4 \\ 17 & 19 & 18 & 17 & 16 & 15 & 9 & 2 \\ 17 & 20 & 19 & 18 & 17 & 16 & 18 & 37\end{array}$

$\begin{array}{llllllll}64 & 79 & 77 & 73 & 67 & 63 & 60 & 73\end{array}$

$\begin{array}{rrrrrrrr}64 & 77 & 75 & 73 & 71 & 69 & 49 & 21 \\ 64 & 77 & 75 & 73 & 70 & 68 & 46 & 7\end{array}$

$\begin{array}{llllllll}64 & 80 & 79 & 76 & 72 & 70 & 88 & 168\end{array}$

$\begin{array}{rrrrrrrr}64 & 80 & 80 & 78 & 74 & 71 & 78 & 103 \\ 64 & 84 & 89 & 84 & 79 & 74 & 70 & 79 \\ 64 & 80 & 83 & 78 & 72 & 68 & 63 & 73 \\ 64 & 99 & 96 & 90 & 85 & 80 & 72 & 81 \\ 64 & 120 & 116 & 111 & 105 & 100 & 94 & 95\end{array}$

\section{B. Bound tests}

B1. Real GDP growth is at historical average minus one standard deviations in 2011-2012 B2. Primary balance is at historical average minus one standard deviations in 2011-2012

B3. Combination of B1-B2 using one half standard deviation shocks

B4. One-time 30 percent real depreciation in 2011

B5. 10 percent of GDP increase in other debt-creating flows in 2011

$\begin{array}{llllllll}3 & 4 & 4 & 6 & 5 & 5 & 4 & 5 \\ 3 & 4 & 4 & 6 & 6 & 5 & 4 & 4 \\ 3 & 4 & 4 & 6 & 5 & 5 & 4 & 4 \\ 3 & 5 & 6 & 7 & 7 & 7 & 6 & 6 \\ 3 & 4 & 6 & 7 & 7 & 7 & 5 & 6\end{array}$

Sources: Country authorities; and staff estimates and projections.

1/ Assumes that real GDP growth is at baseline minus one standard deviation divided by the square root of the length of the projection period.

2/ Revenues are defined inclusive of grants. 
Appendix Figure 2.Rwanda: Indicators of Public Debt Under Alternative Scenarios, 2010-2030 1/
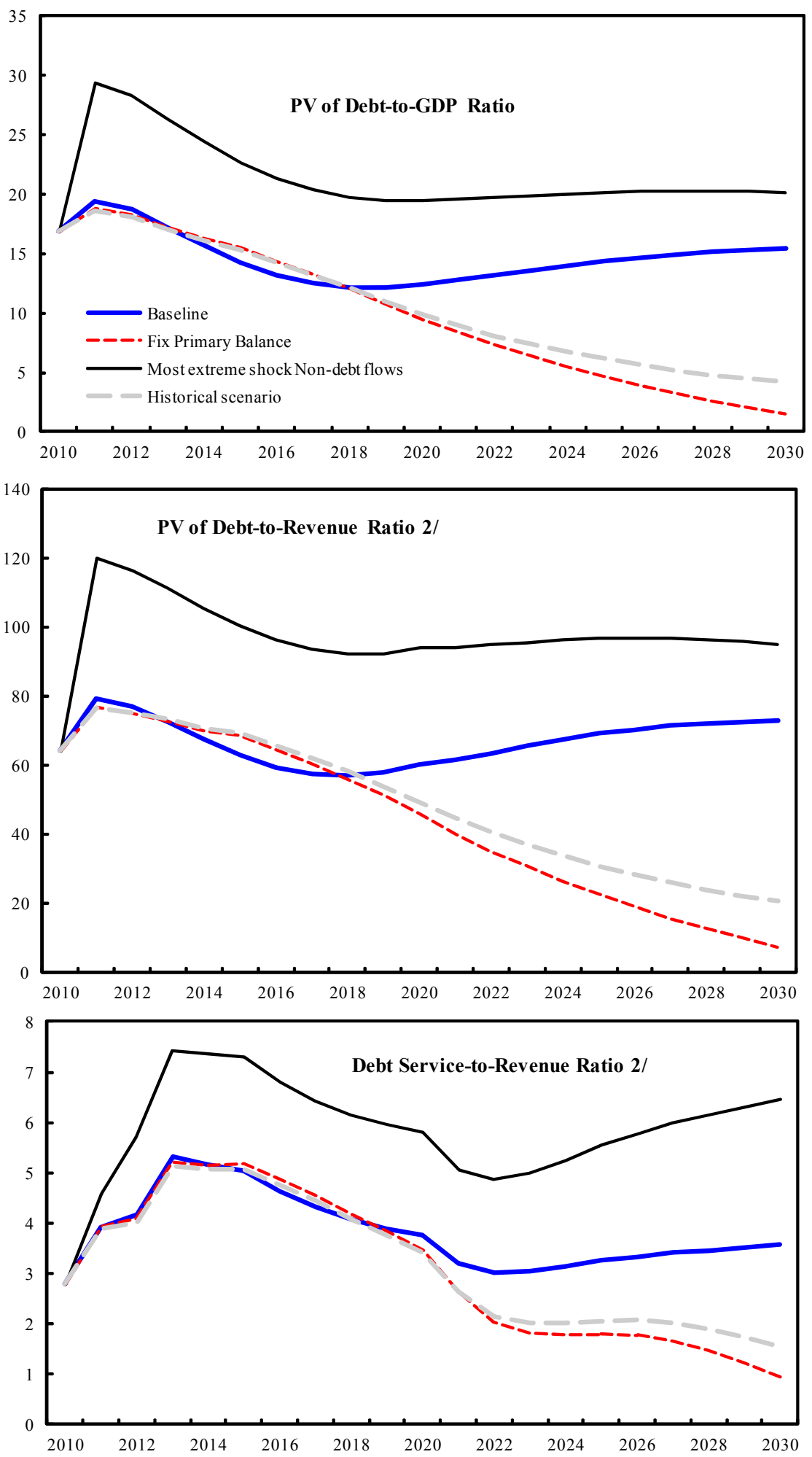

Sources: Country authorities; and staff estimates and projections.

1/ The most extreme stress test is the test that yields the highest ratio in 2020.

2/ Revenues are defined inclusive of grants. 
Press Release No. 10/247

International Monetary Fund

FOR IMMEDIATE RELEASE

Washington, D.C. 20431 USA

June 17, 2010

\section{IMF Executive Board Approves a New Three-Year Policy Support Instrument for Rwanda}

The Executive Board of the International Monetary Fund (IMF) has approved a new threeyear Policy Support Instrument (PSI) for Rwanda. The IMF's framework for PSIs is designed for low-income countries that may not need, or want, IMF financial assistance, but still seek IMF advice, monitoring, and endorsement of their policies. (see Public Information Notice No. 05/145).

The PSI for Rwanda aims to consolidate macroeconomic stability, achieve sustained broadbased growth, while reducing Rwanda's aid dependency. The authorities' program is designed to achieve these objectives by maintaining a sustainable fiscal position; strengthening monetary and exchange rate policies; and supporting growth with structural reforms to diversify the export base and improve the business environment.

Following the Executive Board's discussion of Rwanda on June 16, Mr. Murilo Portugal, Deputy Managing Director and Acting Chair, stated:

"Rwanda has achieved high growth and macroeconomic stability under three successive IMF arrangements. Inflation, though volatile, is now in single digits, international reserves are at comfortable levels, and external debt has been reduced significantly thanks to prudent policies and substantial debt relief.

"Rwanda is making a transition to a new three-year Policy Support Instrument, the natural progression following a successful stabilization program, and given the lack of need for IMF financial support at this time. The macroeconomic priorities and structural reforms in the new program focus on preserving macroeconomic stability, consistent with the authorities' poverty reduction and growth strategy (the EDPRS), while addressing vulnerabilities arising from low levels of fiscal revenues, a narrow export base, high aid dependence and volatile inflation. 
"Growth is underpinned by sound macroeconomic policies, higher investment and commitment to implement a vigorous structural reform agenda. Fiscal reforms focus on enhancing spending efficiency through reforms in public financial management and increasing domestic revenues. Limited use of non-concessional borrowing will allow for scaling up of critical growth-enhancing investments without worsening debt vulnerabilities. Measures to address shortages of skilled labor, expand the export base, and further remove key bottlenecks to doing business are expected to enhance growth acceleration and poverty reduction.

"Financial sector reforms, including building supervisory capacity and improving liquidity management, will further support growth and enhance the effectiveness of monetary policy. In particular, low and stable inflation is expected to remain anchored on the authorities' commitment to greater exchange rate flexibility as well as effective use of available monetary policy instruments", Mr. Portugal added.

ANNEX

\section{Recent Economic Developments}

Growth in Rwanda slowed sharply in 2009 to about 4 percent after growing by 11.2 percent in 2008, as a result of declining commodity prices following the global economic crisis and tight domestic liquidity conditions. Inflation also declined significantly from 22.3 percent in December 2008 to 5.7 percent in December 2009, and further to 3 percent in April 2010, reflecting lower food prices and the slowdown in economic activity.

The medium-term outlook looks favorable and reflects a gradual recovery after the slowdown in 2009. Growth is expected to average 6 percent in 2010-13 supported mainly by the government's investment strategy aimed at alleviating critical infrastructure constraints to exports of goods and services and further enhancing competitiveness. Rwanda will need strong policies that preserve fiscal sustainability and deliver low and stable inflation, and growth-enhancing structural reforms aimed at deepening the financial sector, diversifying export base, and improving the business environment.

\section{Program Summary}

Rwanda's PSI will support the government's program to achieve sustainable broad-based high growth and poverty reduction, in line with the objectives outlined in the government's EDPRS. Fiscal policy will focus on increasing revenue mobilization aimed at reducing Rwanda's aid dependence. Domestic revenue is targeted to increase by 2 percent of GDP over the PSI period, backed up by significant revenue administration reforms. Monetary policy will aim at achieving a 7 percent inflation at end-2010, taking into account expected rise in oil prices, and stabilizing at 5 percent over the medium term. This will be supported 
by a shift towards greater exchange rate flexibility and a more active interbank market, while at the same time maintaining a comfortable level of international reserves that cushion against exogenous shocks. Financial sector reforms, including building supervisory capacity and improving liquidity management, should support growth and enhance the effectiveness of monetary policy.

Rwanda: Selected Economic and Financial Indicators, 2008-15

\begin{tabular}{|c|c|c|c|c|c|c|c|c|}
\hline & $\begin{array}{r}2008 \\
\text { Act. }\end{array}$ & $\begin{array}{r}2009 \\
\text { Est. }\end{array}$ & $\begin{array}{c}2010 \\
\text { Proj. }\end{array}$ & $\begin{array}{c}2011 \\
\text { Proj. }\end{array}$ & $\begin{array}{c}2012 \\
\text { Proj. }\end{array}$ & $\begin{array}{c}2013 \\
\text { Proj. }\end{array}$ & $\begin{array}{c}2014 \\
\text { Proj. }\end{array}$ & $\begin{array}{r}2015 \\
\text { Proj. }\end{array}$ \\
\hline \multicolumn{9}{|l|}{ ¿DP and Prices (percent change) } \\
\hline Real GDP growth & 11.2 & 4.1 & 5.4 & 5.9 & 6.4 & 6.9 & 6.5 & 6.5 \\
\hline Real GDP (per capita) & 8.9 & 2.0 & 3.2 & 3.7 & 4.2 & 4.7 & 4.3 & 4.4 \\
\hline Consumer prices (period average) & 15.4 & 10.4 & 6.4 & 6.5 & 5.5 & 5.0 & 5.0 & 5.0 \\
\hline Consumer prices (end of period) & 22.3 & 5.7 & 7.0 & 6.0 & 5.0 & 5.0 & 5.0 & 5.0 \\
\hline \multicolumn{9}{|l|}{ :xternal sector (percent change) } \\
\hline Terms of trade (deterioration $=-$ ) & -1.4 & -9.9 & 2.9 & -2.8 & -2.2 & -2.3 & -0.9 & 1.1 \\
\hline Real effective exchange rate (end of period; depreciation-) & 22.3 & 1.4 & $\ldots$ & $\ldots$ & $\ldots$ & $\ldots$ & $\ldots$ & $\ldots$ \\
\hline \multicolumn{9}{|l|}{ loney and credit $^{1}$ (percent change) } \\
\hline Broad money (M2) & 24.2 & 13.1 & 12.4 & 12.3 & 12.3 & 12.3 & 12.3 & 12.3 \\
\hline Domestic credit ${ }^{2}$ & 20.5 & 3.8 & 11.0 & 11.4 & 13.7 & 12.6 & 10.5 & 10.2 \\
\hline Credit to the central government $^{2}$ & -18.1 & -0.4 & -2.5 & 0.0 & 0.0 & 0.0 & 0.0 & 0.0 \\
\hline Credit to the economy $y^{2}$ & 38.6 & 4.2 & 13.5 & 11.4 & 13.7 & 12.6 & 10.5 & 10.2 \\
\hline iavings and investment gap (percent of GDP) & -14.4 & -17.3 & -19.3 & -18.6 & -14.8 & -12.9 & -11.6 & -10.6 \\
\hline Gross domestic investment & 23.5 & 21.3 & 22.8 & 22.7 & 21.5 & 20.8 & 20.4 & 20.3 \\
\hline Public & 10.4 & 10.0 & 11.1 & 10.7 & 9.4 & 8.6 & 8.1 & 7.7 \\
\hline Private & 13.1 & 11.3 & 11.7 & 12.0 & 12.1 & 12.2 & 12.3 & 12.6 \\
\hline Vational savings (excluding grants) & 9.1 & 4.0 & 3.5 & 4.1 & 6.6 & 7.8 & 8.9 & 9.7 \\
\hline Public & 0.0 & -2.7 & -3.4 & -2.1 & -2.3 & -1.4 & -1.0 & -0.5 \\
\hline Private & 9.2 & 6.7 & 6.9 & 6.2 & 9.0 & 9.2 & 9.8 & 10.1 \\
\hline \multicolumn{9}{|l|}{ :xternal sector (percent of GDP) } \\
\hline Current account balance (including grants) & -4.9 & -7.3 & -7.9 & -8.8 & -5.4 & -4.4 & -3.7 & -3.5 \\
\hline Current account balance (excluding grants) & -14.4 & -17.3 & -19.3 & -18.6 & -14.8 & -12.9 & -11.6 & -10.6 \\
\hline External debt (end of period) & 14.4 & 14.4 & 16.4 & 19.3 & 19.1 & 18.0 & 16.7 & 15.4 \\
\hline Scheduled debt-service ratio ${ }^{3}$ & 1.3 & 1.8 & 3.0 & 6.3 & 6.9 & 7.8 & 7.4 & 6.7 \\
\hline \multicolumn{9}{|l|}{ jovernment budget and debt (percent of GDP) } \\
\hline Revenue & 14.9 & 12.8 & 12.8 & 13.3 & 13.8 & 14.2 & 14.6 & 14.8 \\
\hline irants & 10.9 & 11.7 & 13.5 & 11.3 & 10.7 & 9.6 & 8.7 & 7.8 \\
\hline Total expenditure and net lending & 25.3 & 25.5 & 27.3 & 26.1 & 25.5 & 24.2 & 23.7 & 23.0 \\
\hline Overall balace (including grants) & 0.4 & -0.9 & -1.0 & -1.5 & -1.0 & -0.4 & -0.4 & -0.3 \\
\hline Overall balace (excluding grants) & -10.5 & -12.6 & -14.5 & -12.8 & -11.7 & -10.0 & -9.1 & -8.2 \\
\hline Stock of domestic debt & 11.8 & 6.8 & 7.7 & $\ldots$ & $\cdots$ & $\cdots$ & $\ldots$ & $\cdots$ \\
\hline \multicolumn{9}{|l|}{ 1emorandum item: } \\
\hline Nominal GDP (billions of Rwanda Francs) & 2,565 & 2,964 & 3,333 & 3,746 & 4,210 & 4,725 & 5,285 & 5,912 \\
\hline Average exchange rate (Rwanda Franc / US\$) & 547 & 565 & 586 & 612 & 633 & 652 & 672 & 692 \\
\hline Average treasury bill yeild (percent) & 6.9 & 8.7 & $\ldots$ & $\ldots$ & $\ldots$ & $\ldots$ & $\ldots$ & $\ldots$ \\
\hline Overall balance of payments (US\$ millions) & 65 & 139 & 4 & -43 & 10 & 1 & 32 & 40 \\
\hline $\begin{array}{l}\text { Gross reserves (in months of imports of goods and } \\
\text { services) }\end{array}$ & 4.8 & 5.1 & 4.8 & 4.7 & 4.7 & 4.4 & 4.3 & 4.2 \\
\hline
\end{tabular}

Sources: Rwandan authorities; and IMF staff estimates and projections.

Projections are based on the program exchange rate of RwF per US dollar of of 571.24.

As a percent of the beginning-of-period stock of broad money.

As a percent of exports of goods and services.

Data from 2009 onwards includes SDR Allocation. 


\section{Statement by Laurean Rutayisire, Executive Director for Rwanda June 16, 2010}

My Rwandan authorities would like to thank staff for the constructive discussions held in Kigali and in Washington, in preparation of the Policy Support Instrument. My authorities would also like to thank the Executive Board and Management for their continued support which has helped Rwanda make important strides in the pursuit of its development goals.

After successfully implementing three successive PRGF arrangements over the past years, Rwanda has demonstrated program ownership and posted strong results on the fronts of macroeconomic stability and output growth, which can fairly qualify the country as a mature stabilizer. Despite the challenges still facing the economy in terms of dependence on foreign assistance and narrow export base, much has been achieved in public financial management, bringing inflation down, building an enabling environment for businesses, enhancing governance, modernizing the economy and reducing poverty. In the PSI framework, my authorities are committed to stepping up their effort in the construction of a broad-based economy capable of posting high and sustained growth rates and reducing poverty further as envisaged in the government's Economic Development and Poverty Reduction Strategy (EDPRS) 2008-2012.

\section{TRACK RECORD AND THE CASE FOR A PSI ARRANGEMENT}

\section{Achievements under past programs}

Over the 1998-2008 period, my authorities have recorded appreciable policy implementation performances. As a result, real GDP growth averaged 8 percent a year during that period, up from 2 percent a year in the 1980s, and higher than the average for SSA countries. Though inflation sometimes peaked up, mainly as a result of exogenous factors, it has currently been brought down to single digits. My authorities have also significantly improved public financial management by strengthening human resource capacities, including in the areas of economic management and budgeting, financial management and reporting, public procurement and budget execution oversight. Fiscal policy was geared towards the overarching goal of increasing the revenue to GDP ratio with the view of reducing the country's high dependence on foreign aid. In that regard, tax administration notably the Rwanda Revenue Authority has made major improvements and my authorities are committed to continuing on the same path.

With the support of their partners, my authorities have also improved overall policymaking, notably in laying down their strategies in many policy areas, such as public investment, medium term debt management, private sector development and financial sector development. As regards debt management, in the face of pressing development needs, my 
authorities have maintained the favorable outlook achieved since reaching HIP completion point and MDRI, which improved the risk of debt distress from high to moderate. By keeping up their debt management guiding principle of ensuring that the financing requirements and repayments are met at least cost for economically and financially viable projects, my authorities have garnered donor support while keeping pace with debt sustainability. Under past programs, my authorities have also strengthened their ability to manage exogenous shocks and crises. In that regard, though the recent global crisis negatively affected exports receipts and FDI, my authorities responded appropriately, including through fiscal adjustment measures.

\section{Recent Economic Developments}

My authorities have maintained a determined good policy implementation as evidenced by the broadly satisfactory performance under the macro framework agreed with Fund staff for 2009/10. Growth slowed as a consequence of the global crisis, from 11.2 percent in 2008 to 6 percent in 2009. Inflation sharply declined from 22.3 percent in December 2008 to 5.7 percent in December 2009, then to 3 percent in April 2010. The fiscal stance is reflecting the slowdown in economic activity, with declining domestic revenue and high levels of spending. My authorities expect the fiscal deficit to narrow as the economy recovers and revenue regains momentum with their measures for the coming period.

My authorities pursued an accommodative monetary policy in 2009 and began to solve the liquidity issue that persisted in 2008, affecting banks' profitability, increasing NPLs and causing a sharp decline in credit growth to the private sector. Bank liquidity improved during 2009, and the initiatives taken by the National Bank of Rwanda (NBR), including the lowering of its policy rate and the swap agreement with the IFC in November 2009, will help provide additional resources for long-term lending to the private sector. As regards the external position, the overall balance of payment benefited from resuming FDI inflows, private-sector external borrowing and SDR allocations, despite the widening of the current account deficit due to underperforming exports. Official reserves increased to a comfortable level of 5.1 months of imports.

\section{GROWTH-ENHANCING POLICIES AND REFORMS}

My Rwandan authorities are determined to build on the progress made over the past years. For my authorities, macroeconomic stability should be maintained and serve as the basis for a more ambitious program that emphasizes bold initiatives and strategies for a sustained and broad-based high growth of 6-7 percent a year. In that regard, they remain committed to the provision of public investment, inducing private investment, as the key driver of the ambitious acceleration of economic growth underpinning the macroeconomic framework of the EDPRS. The policies and reform agenda set forth should cover the PSI period and 
beyond. The government will seek to consolidate macroeconomic stability by: (i) establishing a sustainable fiscal position, with strong revenue efforts needed to reduce Rwanda's aid dependency; (ii) strengthening monetary and exchange rate policies to ensure low and stable inflation; and (iii) pursuing structural reforms that deepen the financial sector, diversify the export base, and improve the business environment.

\section{Pursuing fiscal consolidation}

My authorities' fiscal policy will balance the competing objectives of supporting the growth and poverty reduction goals as envisaged in the EDPRS while preserving fiscal and debt sustainability over the medium term. The effort should bring the overall fiscal deficit (including grants) from 3.6 percent of GDP in 2010/11 to less than 1 percent of GDP by 2012/13. To that end, revenue is set to increase by 2 percent of GDP over the PSI period. The strategy also envisages prioritization of expenditures to accommodate additional spending associated with some of the six government strategic investment projects. Further reforms in revenue administration and in PFM will underpin the medium-term fiscal performance.

\section{Strengthening monetary and exchange rate policies}

My authorities intend to strengthen monetary policy with the view to better addressing issues such as liquidity shortages and inflation concerns that persisted in the past. The inflation target for 2010 is set at 7 percent, and should stabilize at 5 percent over the medium term. Accordingly, some changes have been introduced to make monetary policy more proactive. The NBR's Monetary Policy Committee will now meet quarterly and the key repo rate will be reviewed regularly to respond to changing fundamentals. In addition, the NBR will improve communication around monetary policy decisions with the view to helping shape expectations of markets and the public. My authorities are also working to move towards greater exchange rate flexibility and to enhance interbank market.

\section{Scaling up infrastructure investments while maintaining debt sustainability}

My Rwandan authorities' strong belief that underpinned the EDPRS and development strategies for years is that the construction of a flourishing and broad-based economy that raises the national income and provides opportunities for everyone is the ultimate means to reduce poverty in a sustainable manner. That is why the scaling up of economic infrastructure investments to foster growth is key and remains an unwavering policy stance of my authorities in the dialogue with partners. While being cognizant of the need to maintain debt ratios at sustainable levels, my authorities have continuously drawn the attention of their partners on the fact that debt management concerns should accommodate countries' pressing and huge needs of infrastructures and capital goods. 
Two of my authorities' strategic investment projects, the expansion of Rwandair (the national air carrier) and the building of the Kigali International Convention Complex, fall under the solutions to infrastructure constraints to a service sector-led growth. While these projects will require some nonconcessional borrowing, staff assessed that it will not unduly increase Rwanda's risk of debt distress. In addition, the borrowing takes into account the limited availability of concessional financing, a shallow domestic capital market, and the need to avoid crowding out the private sector. As regards the viability of the projects, the authorities have conducted feasibility studies, with assistance from international consultants, which show they are expected to have rates of return above financing costs.

\section{Deepening structural reforms over the medium term}

Beyond the structural measures to be implemented in 2010/11, my authorities are planning further structural reforms over the medium term in revenue administration, PFM, financial sector, economic integration within the EAC, and export promotion. Revenue administration measures will include introducing $e$-taxes at RRA headquarters, and rolling out to district and provincial offices, automating the tax audit function by introducing computer-based risk assessments and case selection, and automating collection of social security contributions. PFM will be improved in priority areas such as rolling out of the Integrated Financial Management Information System (IFMIS) core modules to all budget agencies; further strengthening the MTEF through end-user sensitization; continuing fiscal decentralization; and providing further training for procurement officers.

In the financial sector, my authorities will enforce full compliance with the 25 Basle core principles as well as standardization of legal and regulatory framework across EAC, both by December 2012. Their agenda also encompasses measures to better supervise the MFIs and deepen the financial sector, with the view to increasing the financial inclusion rate from 21 percent in 2008 to 30 percent by 2012. My authorities intend to become full member of the EAC to foster economic integration within the region. They are participating in the ground work on macroeconomic convergence criteria prior to the establishment of a monetary union. As regards export promotion, my authorities, through the Rwanda Development Board, are preparing a Comprehensive National Export Diversification Strategy and action plan to be submitted to Cabinet by end-August 2010. The strategy focuses on increasing the value added of existing export sectors and developing new products and services centered around six clusters, including fruits and vegetables processing, specialized tourism especially business tourism, and information technologies. The strategy will also address bottlenecks to make local products more competitive. 


\section{CONCLUSION}

The appreciable results achieved by my authorities in macroeconomic stability and the structural transformation of the Rwandan economy over the past decade have set the stage for more ambitious goals in terms of policymaking for broad-based growth and further poverty reduction. The authorities' program for the period 2010-13, underpinned by their core strategies set forth in the EDPRS goes in that direction. The key pillars of this program evolve around maintaining macroeconomic stability, furthering fiscal consolidation to reduce aid dependency, deepening the financial sector as a strong fuel to private sector activity, and strengthening structural reforms to improve the business climate and broaden the economic base.

With the assistance of their development partners, my authorities believe that Rwanda will continue to make progress towards its main goal of becoming an emerging economy in the near future. In that line, my Rwandan authorities would like to request the support of the Board for a three-year PSI to anchor macroeconomic policies, promote sustained and broadbased growth and make further progress in poverty reduction. 